University of Tennessee Health Science Center

UTHSC Digital Commons

$12-2009$

\title{
Structural Characterization of the C-terminus of the Cochlear Motor Protein Prestin: Intrinsic Disorder Linked to Voltage Sensor Function
}

Michael S. Podgorski

University of Tennessee Health Science Center

Follow this and additional works at: https://dc.uthsc.edu/dissertations

Part of the Medical Cell Biology Commons, and the Speech and Hearing Science Commons

\section{Recommended Citation}

Podgorski, Michael S. , "Structural Characterization of the C-terminus of the Cochlear Motor Protein Prestin: Intrinsic Disorder Linked to Voltage Sensor Function" (2009). Theses and Dissertations (ETD). Paper 215. http://dx.doi.org/10.21007/etd.cghs.2009.0249.

This Dissertation is brought to you for free and open access by the College of Graduate Health Sciences at UTHSC Digital Commons. It has been accepted for inclusion in Theses and Dissertations (ETD) by an authorized administrator of UTHSC Digital Commons. For more information, please contact jwelch30@uthsc.edu. 


\title{
Structural Characterization of the C-terminus of the Cochlear Motor Protein Prestin: Intrinsic Disorder Linked to Voltage Sensor Function
}

\begin{abstract}
The sensitivity of mammalian hearing relies upon the ability to amplify sound. Outer hair cells (OHCs) in the cochlea have motile properties that support this function. The motor protein called prestin resides in the lateral wall of the OHCs. Prestin undergoes voltage-dependent conformational changes, which correlates to the movement of charge through the membrane or non-linear capacitance (NLC). This property underlies $\mathrm{OHC}$ electromotility. A mechanistic understanding of prestin's function remains unknown. Previous research has tied prestin's C-terminus (residues 499-799) to the voltage-dependent events that occur in the membrane. The folding and biochemical properties of prestin's C-terminus were characterized. This information was used to probe the function of the C-terminus.
\end{abstract}

Sequence analysis and limited proteolysis indicated that a 60-70 residue loop partitions the sulfate transporter and anti-sigma factor (STAS) domain in the C-terminus. To investigate the structural and functional role of the 'loop' (residues 571-635), the sequence was deleted $(\Delta)$ from prestin cDNA and transfected into HEK 293T cells. Whole-cell voltage clamp experiments confirmed that prestin $\Delta 571-635$ exhibited NLC; however, the voltage-capacitance curve had an increased slope-factor compared to wild type. This result suggested that the mutation affected the protein's voltage sensor. The fact that the deletion protein exhibited NLC implies that the mutation did not adversely affect the structure. It was hypothesized that only a small subset of residues, attributed to the STAS domain, contribute to the structure in prestin's $\mathrm{C}$-terminus. To characterize the $\mathrm{C}$-terminus structure, a series of mutant proteins were made with sequence deleted from the regions bordering the STAS domain. The implication being that the deletion would destabilize the protein if it disrupted important structure. On that premise, nuclear magnetic resonance spectroscopy (NMR), and analytical ultracentrifugation were used to characterize the folding and stability of the deletion mutants.

The results showed that the deletion mutants existed as folded, monomeric proteins in solution. Interestingly, several of the proteins displayed an ensemble of folding conformations that ranged from compact/globular to prolate/ellipsoid. Based on the deletions, the data suggests that the STAS domain consists of two subdomains (STAS A and STAS B) separated by a loop approximately 65 residues long. To understand the basis of the observed conformations, surface plasmon resonance (SPR) imaging determined whether binding occurs between the two subdomains. The data showed that the STAS A and $B$ subdomains bind in a specific and reversible manner characterized by a slow on-rate with weak affinity. NMR data showed chemical shifts consistent with a folded protein; however, differences of peak intensity suggested that movement occurred within the structure of the C-terminus. The observations from the NMR and SPR studies appeared to be consistent with conformations observed from the analytical ultracentrifugation. In summary, the data suggests that the C-terminus contributes to the voltage sensor function. These behaviors of the protein in solution appear to be an innate feature of the C-terminus and may define the unique properties of prestin within the SLC26 protein family.

\section{Document Type}

Dissertation

\section{Degree Name}

Doctor of Philosophy $(\mathrm{PhD})$

\section{Program}

Interdisciplinary Program 


\section{Research Advisor}

Jian Zuo, Ph.D.

\section{Keywords}

C-terminus, conformation, disorder, prestin, STAS domain, voltage sensor

\section{Subject Categories}

Medical Cell Biology | Medical Sciences | Medicine and Health Sciences | Speech and Hearing Science 


\title{
Structural Characterization of the C-terminus of the Cochlear Motor Protein Prestin: Intrinsic Disorder Linked to Voltage Sensor Function
}

\author{
A Dissertation \\ Presented for \\ The Graduate Studies Council \\ The University of Tennessee \\ Health Science Center \\ In Partial Fulfillment \\ Of the Requirements for the Degree \\ Doctor of Philosophy \\ From The University of Tennessee
}

By

Michael S. Podgorski

December 2009 
Copyright (C) 2009 by Michael S. Podgorski All rights reserved 


\section{Dedication}

To the ones before this point: this undertaking is dedicated to my grandparents who understood that careful observation is often the most useful way to educate oneself. My big brother, little brother, and of course, mom and dad always inquire about the weather in my local. I think that is one of the best metaphors for family. To my wife Ying, baby Flynn, and Ehlana who keep my grown self going ever after with their love. To them, I can never show too much gratitude. 


\section{Acknowledgements}

Much of the patience required to become a scholar is a learned skill. Perhaps, the individuals with the most forbearance that one encounters on the journey are the members of the doctoral committee, who bravely lend their support in the face of student angst. Particularly, I would like to show my deep appreciation to Dr. Jian Zuo for serving as my mentor and taking on the difficult task of cultivating my intellect. I also thank Dr. Dianna Johnson for teaching me the importance of good communication in science. In hindsight, Dr. Linda Hendershot, Dr. James Morgan, and Dr. Stephen White taught me more through the Socratic method than by any dialogue. Such questions are the heart of research. I offer my humble thanks to my lab mates. In particular, Dr. Tetsuji Yamashita and Dr. Thomas Weber provided the friendship and beverages that kept things in perspective. Dr. Danny Huang was a gentleman, a scholar, my best man, and the greatest roommate ever. Dr. Amanda Nourse and Brett Waddell believed in the my project despite the difficulties and my foolish ideas. Dr. Richard Kriwacki, Dr. Ou Li, Dr. Steve Otieno, Cheon-Gil Park, and all of the members of the Kriwacki laboratory balanced my enthusiasm with sound judgment and guidance to which I am grateful. I would also like to recognize everyone at St. Jude Children's Research Hospital and the University of Tennessee who counseled, guided, or simply had the patience to listen to my questions. My wife and family deserve special gratitude in that regard. I would also like to distinguish the American Lebanese and Syrian Associated Charities and the National Institutes of Health for their financial support, which is the lifeblood of any research.

Finally, I would like to acknowledge the kindness of the following individuals for their technical contributions. Dr. Jie Fang lent his expertise to the electrophysiology experiments, Dr. Amanda Nourse to the analytical ultracentrifugation and light scattering, Dr. Li Ou and Dr. Matt Revington to the NMR studies, and Dr. Tetsuji Yamashita to the yeast two-hybrid experiments. 


\begin{abstract}
The sensitivity of mammalian hearing relies upon the ability to amplify sound. Outer hair cells (OHCs) in the cochlea have motile properties that support this function. The motor protein called prestin resides in the lateral wall of the OHCs. Prestin undergoes voltage-dependent conformational changes, which correlates to the movement of charge through the membrane or non-linear capacitance (NLC). This property underlies $\mathrm{OHC}$ electromotility. A mechanistic understanding of prestin's function remains unknown. Previous research has tied prestin's C-terminus (residues 499-799) to the voltage-dependent events that occur in the membrane. The folding and biochemical properties of prestin's C-terminus were characterized. This information was used to probe the function of the C-terminus.
\end{abstract}

Sequence analysis and limited proteolysis indicated that a 60-70 residue loop partitions the sulfate transporter and anti-sigma factor (STAS) domain in the C-terminus. To investigate the structural and functional role of the 'loop' (residues 571-635), the sequence was deleted $(\Delta)$ from prestin cDNA and transfected into HEK 293T cells. Whole-cell voltage clamp experiments confirmed that prestin $\triangle 571-635$ exhibited NLC; however, the voltage-capacitance curve had an increased slope-factor compared to wild type. This result suggested that the mutation affected the protein's voltage sensor. The fact that the deletion protein exhibited NLC implies that the mutation did not adversely affect the structure. It was hypothesized that only a small subset of residues, attributed to the STAS domain, contribute to the structure in prestin's C-terminus. To characterize the $\mathrm{C}$-terminus structure, a series of mutant proteins were made with sequence deleted from the regions bordering the STAS domain. The implication being that the deletion would destabilize the protein if it disrupted important structure. On that premise, nuclear magnetic resonance spectroscopy (NMR), and analytical ultracentrifugation were used to characterize the folding and stability of the deletion mutants.

The results showed that the deletion mutants existed as folded, monomeric proteins in solution. Interestingly, several of the proteins displayed an ensemble of folding conformations that ranged from compact/globular to prolate/ellipsoid. Based on the deletions, the data suggests that the STAS domain consists of two subdomains (STAS A and STAS B) separated by a loop approximately 65 residues long. To understand the basis of the observed conformations, surface plasmon resonance (SPR) imaging determined whether binding occurs between the two subdomains. The data showed that the STAS A and B subdomains bind in a specific and reversible manner characterized by a slow on-rate with weak affinity. NMR data showed chemical shifts consistent with a folded protein; however, differences of peak intensity suggested that movement occurred within the structure of the C-terminus. The observations from the NMR and SPR studies appeared to be consistent with conformations observed from the analytical ultracentrifugation. In summary, the data suggests that the $\mathrm{C}$-terminus contributes to the voltage sensor function. These behaviors of the protein in solution appear to be an innate feature of the $\mathrm{C}$-terminus and may define the unique properties of prestin within the SLC26 protein family. 


\section{Table of Contents}

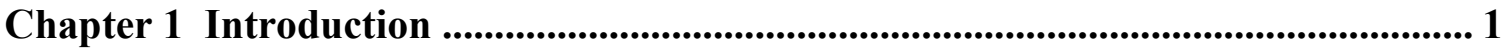

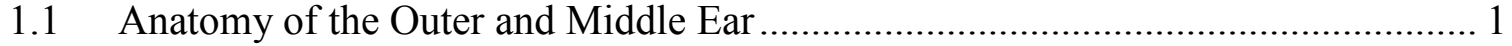

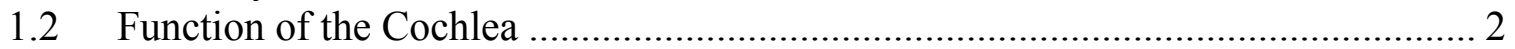

1.2.1 Architecture of the Cochlear Structures ...................................................... 2

1.2.2 The Organ of Corti Is the Site of Signal Transduction................................. 3

1.2.3 Cochlea Function as a Frequency Analyzer and Amplifier ........................ 4

1.2.4 OHC Electromotility Is the Cochlear Amplifier ..................................... 5

1.3 Prestin Function and Electromotility.............................................................. 7

1.3.1 Prestin's Relationship to Amplification and Electromotility ...................... 7

1.3.2 Prestin Function Derives from the OHC Membrane.................................. 9

1.4 Passive Function of Prestin and OHC Stiffness ............................................... 12

1.5 C-terminus Relationship to Prestin Function .................................................... 14

1.5.1 Mechanism of Prestin-prestin Interaction Unknown............................... 14

1.5.2 Specific Functional Role of the C-terminus Is Unclear ............................. 14

1.5.3 SLC26 Anion Transporters and Sulfate Transporters .............................. 15

1.6 C-terminal STAS Domain Evolved from the Prokaryotic SpoIIaa Protein ........... 17

1.7 Testable Objectives and Project Strategy ........................................................ 19

Chapter 2 Materials and Methods.................................................................................... 22

2.1 Sequence Alignment, Secondary Structure, and Disorder Prediction ................... 22

2.2 Preparation of DNA Constructs for Protein Expression ..................................... 22

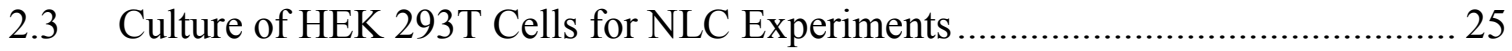

2.3.1 Culture from Frozen Stock ...................................................................... 25

2.3.2 Ethanol Precipitation of DNA for HEK 293T Transfection....................... 25

2.3.3 Transfection of HEK 293 Cells.............................................................. 30

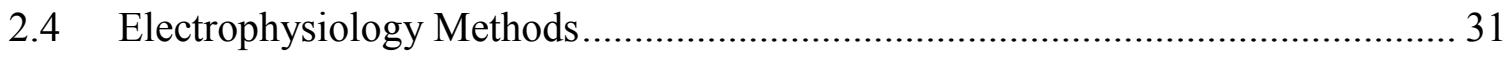

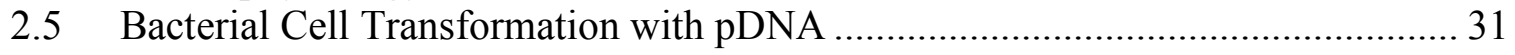

2.6 Expression and Purification of Recombinant C-terminus Proteins ....................... 32

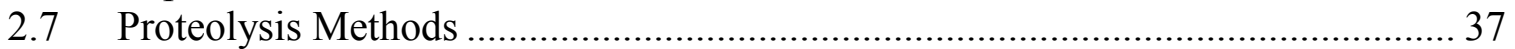

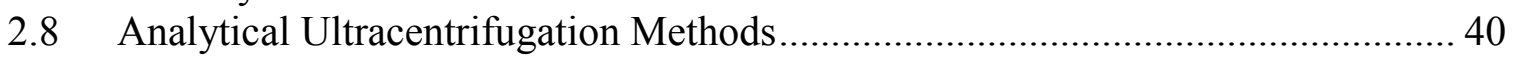

2.9 Size-exclusion Multi-angle Laser Light Scattering Methods ............................... 41

2.10 Nuclear Magnetic Resonance Spectroscopy and Circular Dichroism Methods.... 42

2.11 Surface Plasmon Resonance and Yeast Two-Hybrid Methods ........................... 42

Chapter 3 Functional Test and Characterization of the Disordered Loop .............. 44

3.1 Residues 571-635 Contribute to Prestin's Voltage Sensor .................................. 44

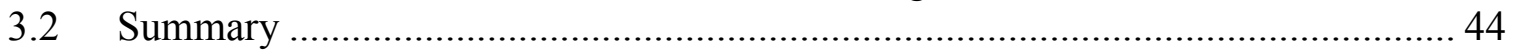

Chapter 4 Insights to C-terminus Folding and Stas Domains Boundaries............... 47

4.1 Deletion Mutants Used to Study Folding ...................................................... 47

4.2 Limited Proteolysis Supports the Disorder Predicted for Residues 571-635 ........ 47

4.3 C-terminus Is Monomeric and Adopts Multiple Conformations .......................... 57 
4.4 C-terminus Is Folded but the Structure Difficult to Resolve....................................66

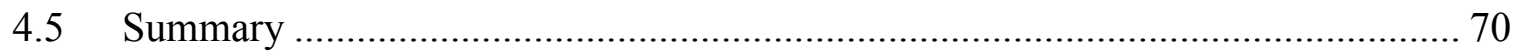

Chapter 5 STAS A-B Interaction May Underlie C-terminus Conformations .......... 71

5.1 Insights to the Different Conformations from Binding Kinetics........................... 71

5.2 Yeast Two-Hybrid Shows Binding Can Occur in the Cell ..................................... 71

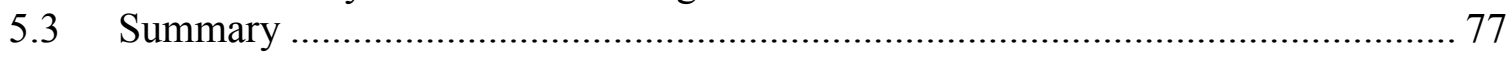

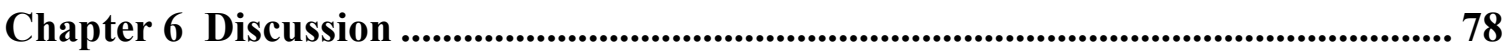

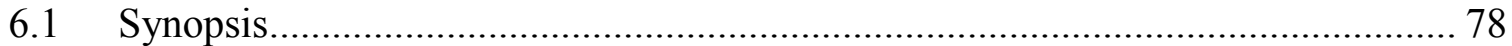

6.2 C-terminus Unlikely to Have a Role Supporting Prestin-prestin Interaction......... 78

6.3 C-terminus Appears to Adopt Multiple Conformations in Solution ...................... 79

6.4 Prestin's STAS Composed of Two Subdomains................................................. 81

6.5 C-terminus Loop Contributes to Prestin's Voltage Sensor ................................... 82

6.6 'MARS' Model Incorporating the C-terminus with the Area Motor Model.......... 84

6.7 Future Studies of the Mechanism Behind the Voltage Sensor with Loop .............. 87

6.7.1 Loop Chimaeras to Identify Functional Differences between Species ...... 87

6.7.2 Relationship between Charged Residues in the Loop and Chloride .......... 87

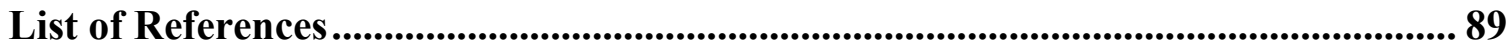

Appendix A Figures of Ear Anatomy .................................................................................. 97

Appendix B Buffers, Solutions, and Media …................................................................... 100

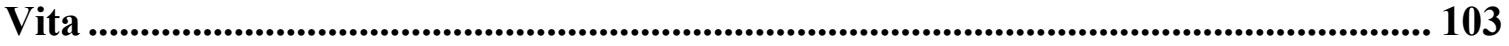




\section{List of Tables}

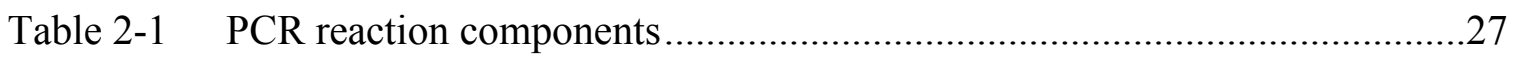

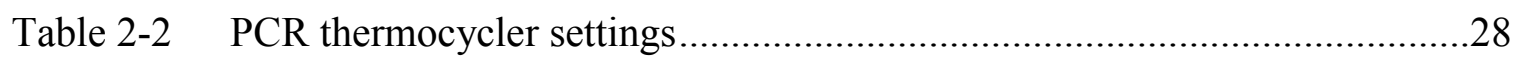

Table 2-3 Oligonucleotide primers used for prestin constructs ..............................29

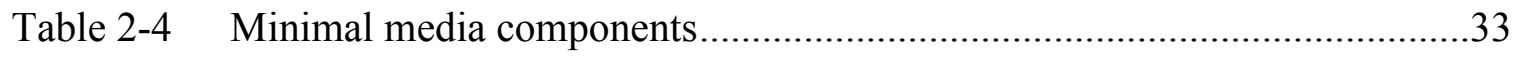

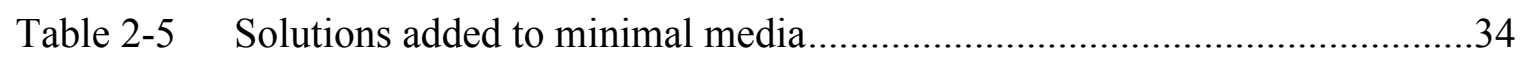

Table 2-6 Predicted bio-physical features of C-terminus proteins ................................39

Table 3-1 NLC data for wild type and $\Delta 571-635$ prestin molecules .........................46

Table 4-1 Proteins used to study the C-terminus structural properties .......................49

Table 4-2 Edman degradation sequence for trypsin cut-sites....................................52

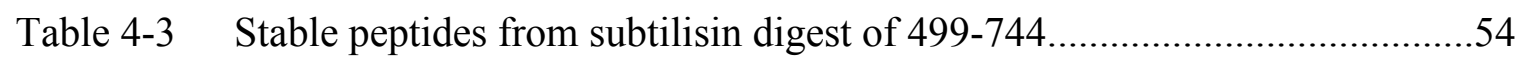

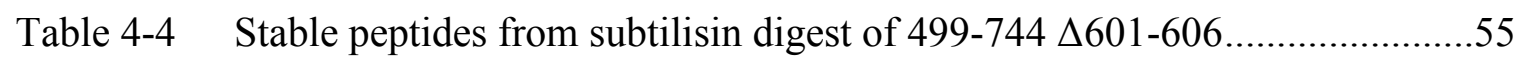

Table 4-5 Summary of SV data for C-terminus proteins .........................................59

Table 5-1 Sequence of biotinylated peptides used in SPR studies............................72

Table 5-2 STAS A peptide and STAS B 613-744 SPR reversible binding data...........74

Table 5-3 A 512-555 peptide and STAS B 613-744 Mono Q fraction SPR data .........75 


\section{List of Figures}

Figure 1-1 Overview of OHC motility generating cochlear amplification.....................6

Figure 1-2 Voltage-dependence of prestin electromotility within the membrane ...........8

Figure 1-3 Charge movement and voltage gating in prestin.....................................11

Figure 1-4 Model of prestin as a membrane-bound area motor .................................13

Figure 1-5 Topology and sequence features of prestin...........................................16

Figure 1-6 Alignment of STAS domains suggests functional differences exist.............18

Figure 1-7 Secondary structure and disorder predictions for the C-terminus ...............20

Figure 2-1 Schematic of pDNA vectors and restrictions sites used in cloning .............23

Figure 2-2 Two-round PCR method used to mutate prestin DNA ..............................24

Figure 2-3 Agarose gel of the PCR products combined to delete 'loop' DNA ..............26

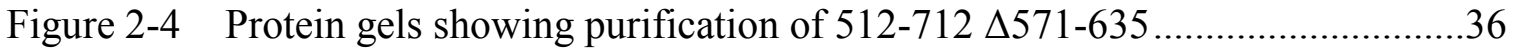

Figure 2-5 Intact mass used to confirm the identity of purified proteins .......................38

Figure 3-1 Full length prestin $\Delta 571-635$ has an altered voltage response....................45

Figure 4-1 Deletion mutants used to isolate the core fold in the C-terminus .................48

Figure 4-2 Primary 'cuts' from trypsin digestion occurred in the loop region..............51

Figure 4-3 Overview of subtilisin cleavage in the C-terminus.................................56

Figure 4-4 SV and SEC-MALS show that the C-terminus does not oligomerize ..........60

Figure 4-5 SE shows that 499-744 $\Delta 571-635$ and 499-744 are monomers..................61

Figure 4-6 2D contour plots: 499-744, 526-744 polar, and 499-744 $\Delta 601-618 \ldots \ldots \ldots \ldots . .63$

Figure 4-7 2D contour plots: 499-744 $\Delta 571-635$ and 526-744 $\Delta 571-635 \ldots \ldots \ldots \ldots \ldots \ldots \ldots . . .64$

Figure 4-8 2D contour plots: 499-709 $\Delta 571-635,512-712 \Delta 571-635$, and STAS B ....65

Figure 4-9 Thermal denaturation of 499-744 $\Delta 571-635$ and 512-712 $\Delta 571-635 \ldots \ldots \ldots . .67$ 
Figure 4-10 C-terminus is folded but structure cannot be resolved by NMR

Figure 4-11 C-terminus proteins appear to have similar secondary structure .69

Figure 5-1 Surface plasmon resonance interaction between STAS A and B ...............73

Figure 5-2 Yeast two-hybrid showing interaction between 499-564 and 639-744 „.......76

Figure 6-1 Diversity of charged residues in the loop of prestin species.......................83

Figure 6-2 MARS model of prestin 'area' changes in the membrane ..........................85

Figure A-1 Pathway of sound into the cochlea................................................... 97

Figure A-2 Cross-section of the cochlea showing the key sensory structures ...............98

Figure A-3 Tonotopic layout of the hair cells along the basilar membrane ..................99 


\section{List of Abbreviations}

$\Delta$. $\Delta 571-635$

$\Omega$

A

AUC

$\mathrm{B}$

CAPS

$\mathrm{C}_{\text {lin }}$

$\mathrm{C}_{(V)}$

$\mathrm{dB}$

DTT

E.coli

$f / f_{0, w}$

GST

IHC

$\mathrm{kDa}$

$\mathrm{mA}$

MW

Ni-NTA

NLC

NMR

$\mathrm{OHC}$

pDNA

PMSF

$\mathrm{Q}_{\max }$

ref.

rpm

$s$

$s_{20}$

$\mathrm{S}$

SEC-MALS

SPR

STAS

SulP

$\mathrm{V}_{\text {peak }}$

$w$.

$6 \mathrm{x}-\mathrm{His}$ sequence deletion deletion of residue 571-635 from the C-terminus loop ohms resistance

A subdomain of STAS domain analytical ultracentrifugation B subdomain of STAS domain 3-(Cyclohexylamino)-1-propanesulfonic acid .linear capacitance non-linear capacitance decibel dithiothreitol Escherichia coli weight-averaged frictional ratio glutathione s-transferase inner hair cell kilodalton milli amperes .molecular weight nickel-nitrilotriacetic acid non-linear capacitance nuclear magnetic resonance outer hair cell plasmid DNA phenylmethylsulphonyl fluoride maximum nonlinear charge transfer relative centrifugal force revolutions per minute sedimentation coefficient (units of seconds) s-value from contour plots; s vs. $f / f_{0}$ distribution $\left(c\left(s, f / f_{0}\right)\right.$ analysis

Svedberg unit ( $10^{-13}$ seconds) size exclusion chromatography-multi angle light scattering ..surface plasmon resonance sulfate transporter and anti-sigma sulfate Permease .voltage at peak capacitance water as the solvent in AUC calculations six histidine affinity purification tag 


\section{Chapter 1 Introduction}

\subsection{Anatomy of the Outer and Middle Ear}

The ear consists of three distinct anatomical regions called the outer, middle, and inner ear (see Figure A-1). Together these areas function as a bio-physical relay system conducting acoustic energy to the sensory cells in the cochlea. Each part has features that are specialized to preserve the integrity of the sound as it travels to the sensory cells. The description of the anatomy comes from the sources indicated [1-3].

The outer ear consists of the pinna, an exterior flap of cartilaginous tissue with rounded contours, and the ear canal. These structures can be thought of as the auditory systems 'pre-amplifier', which enhances the ability to detect sound. The pinna or auricle behaves like a funnel that gathers and directs sound into the ear. The configuration also helps to filter sounds that are more complex. In particular, high frequency sounds tend to bounce off the pinna's contours, delaying entry into the canal, and causing certain frequencies to fall out phase, which cancels out the signal. From the pinna, sound passes into a hollow tube called the ear canal or external auditory meatus which narrows in diameter until the tympanic membrane. The tapering of the canal helps to passively amplify incoming sound by 30-100 times.

The middle ear is composed of the tympanic membrane or eardrum, which is connected to three tiny bones or ossicles called the malleus, incus, and stapes. Collectively, these structures transfer the sound stimulus into the cochlea and help to amplify the intensity of the signal. The tympanic membrane forms the interface between air and bone. Sound hitting the membrane causes it to vibrate. Direct contact with the malleus transfers the mechanical energy through the ossicles, onto the oval window, and into the fluids in the cochlea.

Several features of the middle ear's anatomy help to maintain the sound energy carried to the cochlea. The tympanic membrane has a large surface area in comparison to the diameter of ossicles and the oval window. The size difference effectively concentrates the pressure from the sound wave assuring that the majority of the energy goes to the bones. Pressure is related the applied force divided by the surface area. This feature minimizes the energy reflected away from the membrane, which occurs due to the transfer between media (air to bone). The ossicles behave like a lever and fulcrum in series that compounds the amount of applied energy when engaged. Together, these features amplify sound to overcome impedance and dampening caused by the cochlear fluids viscosity. The added energy matches the impedance difference avoiding distortions.

The inner ear contains the vestibular organ of balance and the cochlear organ of hearing. Two layers of bone protect the delicate nature of the sensory organs that encompass the inner ear. The temporal bone forms the outermost layer. The bony labyrinth, which houses the vestibular and cochlear organs, lies nestled in the petrous 
portion of the temporal bone. The delicate cellular structures for these organs are found inside a series of interconnected, fluid chambers contained within the calcified shell. The protection afforded by the bone also makes it difficult to gain access and study these systems without causing damage. Two membrane layers line the bony labyrinth.

Periosteum membrane, composed of irregular connective tissue, covers the inside of the bony labyrinth. The membranous labyrinth forms a discrete chamber separate from the periosteum. It follows the contours of the entire bony labyrinth in both the vestibular and cochlear regions. The fluid that fills the membranous labyrinth serves as the medium through which signals are conveyed to the appropriate sensory structures. The vestibular system utilizes the fluid's movement and acceleration to provide spatial information related to the body's position and travel. It consists of the vestibule and the semicircular canals. The vestibule, made-up of the utricle and saccule, monitors the acceleration of movement. It forms an antechamber situated medial to the cochlea's lateral position relative to the bodies midline. The vestibule also contains the oval and round windows. They form the respective entrance and exit for sound passing through the cochlea. The three semicircular canals, located the closest to the midline, branch out from the fluid space of the vestibule. Together, they provide feedback describing rotation and orientation in space.

\subsection{Function of the Cochlea}

\subsubsection{Architecture of the Cochlear Structures}

The cochlea in the mammalian auditory system has evolved with a unique spiralshape. The spatial arrangement of the structures contained inside underlies the ability to hear. The evolutionary reason behind the coiled shape remains uncertain. Among mammals, only monotremes or egg-laying mammals lack a cochlea with a coiled shape [4]. In parallel, birds and reptiles also do not have a cochlea with a spiraled configuration [5]. It has been suggested that the coiled shape developed to accommodate 'greater functionality' in a smaller space. In other words, the spiral shape allowed the cochlea and the basilar membrane to increase in length which endowed sensitivity to a larger range of frequencies [6]. Recent mathematical models of the cochlea indicate that the curvature may increase sensitivity to low frequencies [7].

Three fluid-filled chambers or scalae span the length of the cochlea's spiral from base to apex. In essence, the scalae serve as a highway to convey the acoustic energy to the appropriate place in the cochlea. A cross-section of a single turn in the cochlea highlights the layered arrangement of these chambers (see Figure A-2). The scala vestibuli comprises the upper chamber, the scala tympani the lower, and scala media resides in the middle. The combined volume of all three scala only amounts to one cubic centimeter. The scala vestibuli starts from the oval window at the cochlea's base and extends to the cochlea's apex. An opening at the cochlea's apex, called the helicotrema, connects the scala vestibuli to the scala tympani forming one long, continuous chamber. The scala tympani's path from the apex follows the spiral of the cochlea back to its base. 
The arrangement of the cochlea's chambers helps to maintain distinct ionic differences found in the scala media's fluid compared to the scala vestibuli and tympani. Both the scala vestibuli and tympani contain a high sodium and low potassium electrolyte solution called perilymph. The overall composition of the perilymph resembles extracellular fluid or cerebrospinal fluid. In contrast, the scala media contains a separate fluid called the endolymph which parallels intracellular fluid in its ionic composition. It contains high potassium with low amounts of sodium and calcium. Three different barriers keep the endolymph separate from the perilymph. Reissner's membrane forms the superior border for the scala media and maintains the endolymph separate from the perilymph in the scala vestibuli. The reticular lamina and tight junctions at the apical surface of the hair cells forms the inferior border for the endolymph. Finally, the stria vascularis lines the lateral wall of the periosteum in the scala media. Tight junctions in the stria prevent diffusion with perilymph. The stria reabsorbs sodium and functions as a potassium pump to maintain the high potassium content of the endolymph [8]. The different ionic composition between the perilymph and the endolymph creates a positive potential around $85 \mathrm{mV}$ which serves as an electro-chemical 'battery' to power the transduction of acoustic energy into an electrical signal. The inner and outer hair cells (IHCs and OHCs) in the organ of Corti function as the sensory apparatus where transduction occurs.

\subsubsection{The Organ of Corti Is the Site of Signal Transduction}

Whereas the scalae serve as the pathway for sound energy, the hair cells in the organ of Corti represent the frequency-specific destination. The organ of Corti is located at the lower edge of the scala media. Like the scalae, it stretches the entire length of the cochlea. The endolymph fills the remaining space of the scala media with the stria vascularis framing the outer edge. Together, the organ of Corti and the scala media are named the cochlear duct. The organ of Corti consists of four components to include the sensory apparatus (IHCs and OHCs), various supporting cells, the extracellular basilar, and tectorial membranes. These two membranes frame the upper and lower edges of the organ of Corti creating a sandwich with the various cells types in between (see Figure A-2). The basilar membrane resides at the lower part and separates the scala media from the scala tympani. The tectorial membrane, positioned over the hair cells, forms the uppermost part of the organ of Corti. The arrangement of the various cells can be thought of as following the curvature of the cochlea starting from the inside turn and radiating to the outer edge.

IHCs are the actual sensory cells in the auditory system which detect and convey auditory signals to the brain. The name derives from their location at the inside turn of the cochlear spiral. A single row of IHCs, approximately 3,500 cells total, spans cochlea's length. The cells have a pear-shaped appearance with a rounded bottom that narrows towards the top of the cell. Several rows of 'hair-like' structures called stereocilia cover the apical surface arranged in a 'U-shaped' bundle. Each row has stereocilia of greater length converging to the middle of the ' $U$ '. A network of actin fibers provides structural support within the stereocilia. 
Inner border and inner phalangeal cells closely flank and support the lateral surface of each IHC. The IHC's basal end does not contact the basilar membrane. Afferent nerve fibers and cellular projections from the adjacent supporting cells support the IHCs base on top of the basilar membrane. Lateral to the IHCs, elongated inner pillar and outer pillar cells lean towards each other creating the triangular-shaped tunnel of Corti, which separates the IHCs from the OHCs. Perilymph fills the tunnel hollow space.

The OHCs are located distal to outer pillar cells. As the name suggests, OHCs take a position towards the outer edge of the curvature relative to the IHCs. Three rows of OHCs, around 15,000-16,000 cells, cover the entire cochlea. The OHCs have a cylindrical, polarized shape. The inside of the cell is pressurized [9] which facilitates the cell's function and it provides structural support to the cell's shape [10]. The lateral wall has three layers [11]. The plasma membrane forms the outermost layer. It appears densely packed with an ordered array of unidentified particles [12]. Beneath the plasma membrane, an actin-spectrin cytoskeleton called the cortical lattice forms a flexible net that helps the cell adapt when the internal pressure changes [13, 14]. The innermost layer, called the subsurface cisternae, appears to consist of an endoplasmic-like membrane that's been folded inwards various times and then flattened. The exact structure and function of the subsurface cisternae remains unknown [15]. The overall arrangement of the lateral wall to the pressurized cytoplasm can be thought of as similar to a tire containing a pressurized inner tube. Finally, the OHCs apical surface contains stereocilia similar to IHCs; however, the bundle adopts more of a 'W-shape'. Deiter's cells, located on top of the basilar membrane, cradle the basal end of the OHCs.

\subsubsection{Cochlea Function as a Frequency Analyzer and Amplifier}

The mammalian cochlea is a biomechanical organ. The ability to hear relies upon the integration that occurs between its passive mechanical properties and active processes. The cochlea has two main roles. First, it functions as a frequency analyzer that resolves the individual frequencies that make up complex sounds. This is referred to as tuning or frequency selectivity and serves to increase the range of audible frequencies [16]. Secondly, the cochlea increases the signal to noise ratio. This is called cochlear amplification [17]. In essence, the cochlea receives a sound wave as input and carries out a Fourier transformation to reveal details about the wave's intensity, phase, and timing. Signal transduction at the IHCs transfers this information across the afferent synapse to the cochlear nerve and into the brain. We perceive the outcome of this acoustic spectral analysis as rhythm, tone, and differences in pitch $[18,19]$.

Movement of the stapes transmits sound energy into the cochlea's perilymph, which creates a pressure gradient between the scala vestibuli and tympani. The pressure imbalance squeezes the scala media located in between and generates a traveling wave along the basilar membrane [19]. In the 1950s, Georg von Békésy made the first observations describing the effect of the propagating wave on the basilar membrane. He noticed that the membrane resonated at different locations relative to the specific frequency [20]. In other words, high frequencies caused the basilar membrane to vibrate near the cochlea's base and low frequencies had an affect near the cochlea's apex. 
Intermediate frequencies produced resonance dispersed along the membrane in a gradient relative to the frequency [21]. This association between frequency and location on the basilar membrane is referred to as a tonotopic map [22]. The basilar membrane's stiffness and width change relative to the position in the cochlea. The widest and stiffest part occurs in the high frequency, basal cochlea. At the apex, the basilar membrane narrows and becomes flexible [23]. The tonotopic properties of the basilar membrane are considered as passive or innate to the membrane (see Figure A-3). Evidence for this comes from the fact that von Békésy was able to isolate these properties with cochlea extracted from cadavers.

\subsubsection{OHC Electromotility Is the Cochlear Amplifier}

In 1948, a physicist named Thomas Gold proposed that the ear must have a mechanism to amplify sound waves before reaching the nervous system. He suggested that this must occur to account for the ear's sensitivity and exquisite tuning [24]. Five years later, Hallowell Davis showed that the cochlea contained an element that produced mechanical energy, which in turn boosted hearing sensitivity and resolution. He called this process the 'cochlear amplifier' [17]. Davis' research confirmed the linked nature of frequency selectivity and improvement of the signal to noise ratio. This conclusion led to the idea that amplification derived from a single specialized cell type functioning as the 'power' source [15]. Subsequent research by Brownell pointed to OHCs as the energy source required for amplification. He observed that cultured OHCs displayed movement or motility that resulted from the injection of electrical current into the cells. Under the same conditions, the IHCs did not exhibit motility [25]. Further study, using OHCs isolated from a living cochlea, confirmed that depolarizing currents shortened the $\mathrm{OHC}$ length and increased the cell's diameter $[10,26]$. This activity was later termed electromotility [27]. Parallel research showed that OHC loss directly eliminated hearing sensitivity and tuning [28, 29]. Subsequently, it became accepted that the primary source of energy, which enabled amplification, originated from the OHCs [30].

Both the IHCs and OHCs are positioned above the basilar membrane and directly beneath the tectorial membrane. The basilar membrane's traveling wave lifts the hair cells against the tectorial membrane causing the stereocilia to deflect or bend. This action opens a mechanotransduction channel located in the tips of the stereocilia in both cell types. Potassium flows though the open channel depolarizing the cells which allows them to perform their specific functions [31]. The OHCs serve as the amplifier, while the IHCs are the sensory receptors. The remaining discussion will focus on the OHCs. It is known that the hairbundle can actively bend further in response to the initial event. Hairbundle movement may contribute to amplification; however, the kinetics are not entirely clear [32-34]. The composition of the mechanotransduction channel also remains a mystery [35]. Depolarization of the $\mathrm{OHC}$ activates piezoelectric properties associated with its membrane causing it to contract and elongate along its axis [36, 37]. The cell's length can change up to four percent [38]. Rapid cycles of length change generate mechanical energy, which narrows the basilar membrane's resonant frequency and boosts the signal intensity for the IHCs [12, 39] (see Figure 1-1). In essence, the active movement of the OHCs amplifies the IHCs ability to detect a signal $[17,40]$. 


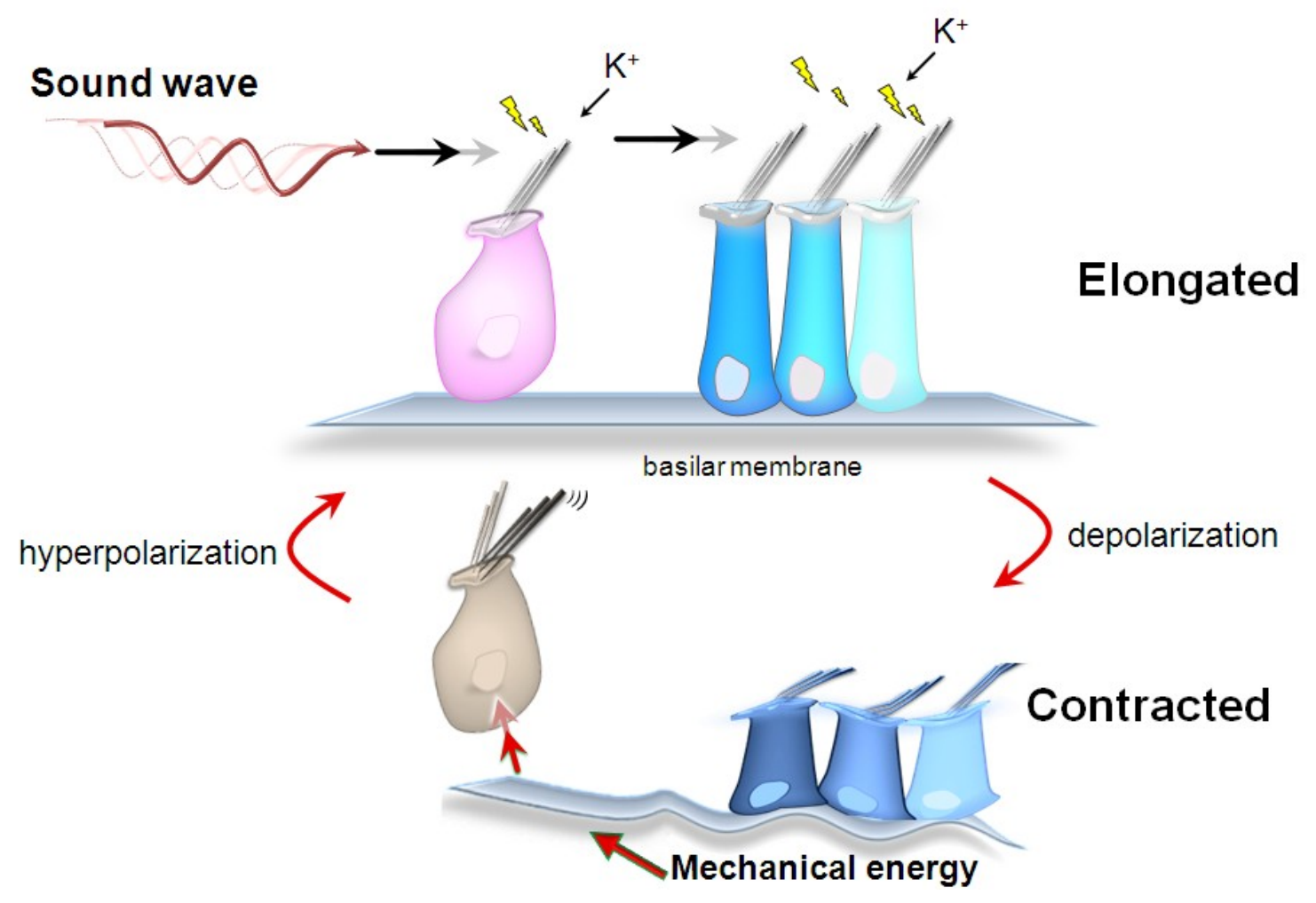

Figure 1-1 Overview of OHC motility generating cochlear amplification

Incoming sound manifests as a pressure wave in the cochlear fluids, which causes the tectorial membrane to bend the stereocilia and open a mechanotransduction channel. The tectorial membrane is not shown for simplicity. The open channel allows the influx of potassium, which generates an inward current and initiates depolarization of the cell. The changing membrane potential induces the $\mathrm{OHC}$ to contract or shorten. Hyperpolarization of the membrane's potential restores the $\mathrm{OHC}$ to a 'resting', elongated form. Multiple cycles of contraction and elongation add energy to the resonant frequency specific to the particular IHCs located next to the active OHCs. IHCs are on the left in pink or brown. $\mathrm{OHCs}$ are on the right in blue. 


\subsection{Prestin Function and Electromotility}

\subsubsection{Prestin's Relationship to Amplification and Electromotility}

As mentioned earlier, Thomas Gold first proposed in 1948 that hearing sensitivity required an amplifier. Over the course of 50 years, research validated his idea and showed that the OHCs performed such a role. Eventually, characterization of OHC electromotility pointed towards the existence of a 'motor' element, located in the cell's lateral wall [41]. It took over 50 years after Gold's statement to pin down the molecular source of the amplifier. In 2000, researchers identified a candidate protein for the motor. They used a subtractive screen comparing mRNA libraries for IHCs and OHCs. They looked for candidates only expressed in OHCs based on the functional differences with IHCs. The results identified a protein that the researchers called prestin. Comparison of prestin's sequence with the proteins, pendrin and DRA (down regulated-in-adenoma), suggested an evolutionary tie to anion transporters [42]. Subsequent analysis classified prestin as a member of the SLC26 anion transporter family [43].

As further proof, details of prestin's expression and function were studied relative to specific aspects of hearing. Northern blot analysis, using tissue from different organs, indicated that expression of prestin occurred solely in the OHCs. Developmentally, expression started at birth and continued for 20 days. This time course overlapped neatly with the post-natal appearance of the 'motor' particles in the membrane and matched the onset of hearing sensitivity [42]. The most significant evidence linking prestin to the motor protein came from tests measuring non-linear capacitance (NLC) and motility. Both abilities are considered hallmarks of OHC electromotility [44]. NLC describes the link between electrical and physical phenomenon in the cell (see Figure 1-2). In practical terms, it derives from the input of additional electrical change between the membrane's dielectric as the voltage potential changes. Charge movement occurs because of structural changes within the cell membrane. These changes to the structure manifest as the physical changes to the cell length. TSA201 cells, transfected with prestin, displayed NLC. Capacitance values quickly changed in a voltage dependent manner similar to measurements in live OHCs. Additional tests were performed using sodium salicylate, which reversibly inhibits NLC in OHCs [45]. The presence of salicylate eliminated charge movement, which could be restored upon washing out the drug. The data from these studies established prestin's functional importance to the motor; however, the results did not preclude the existence of other proteins tied to the motor. The studies could not determine prestin's location in the OHC as an antibody was not available [42].

To provide definitive proof of prestin role, a method was needed to study electromotility in prestin's absence. In 2002, a prestin knockout mouse was generated. The strategy used to create the mouse-targeted deletion of exons 3-7 from prestin's gene. Southern blot analysis and real-time PCR confirmed that prestin expression did not occur in the knockout mouse. Functional tests of the mice looked directly at electromotility in the OHCs and at the downstream affect on hearing to assess prestin's role. Isolated OHCs from the homozygous knockout did not manifest NLC or electromotility. This data provided strong evidence that prestin directly affected the mechanics of amplification. 

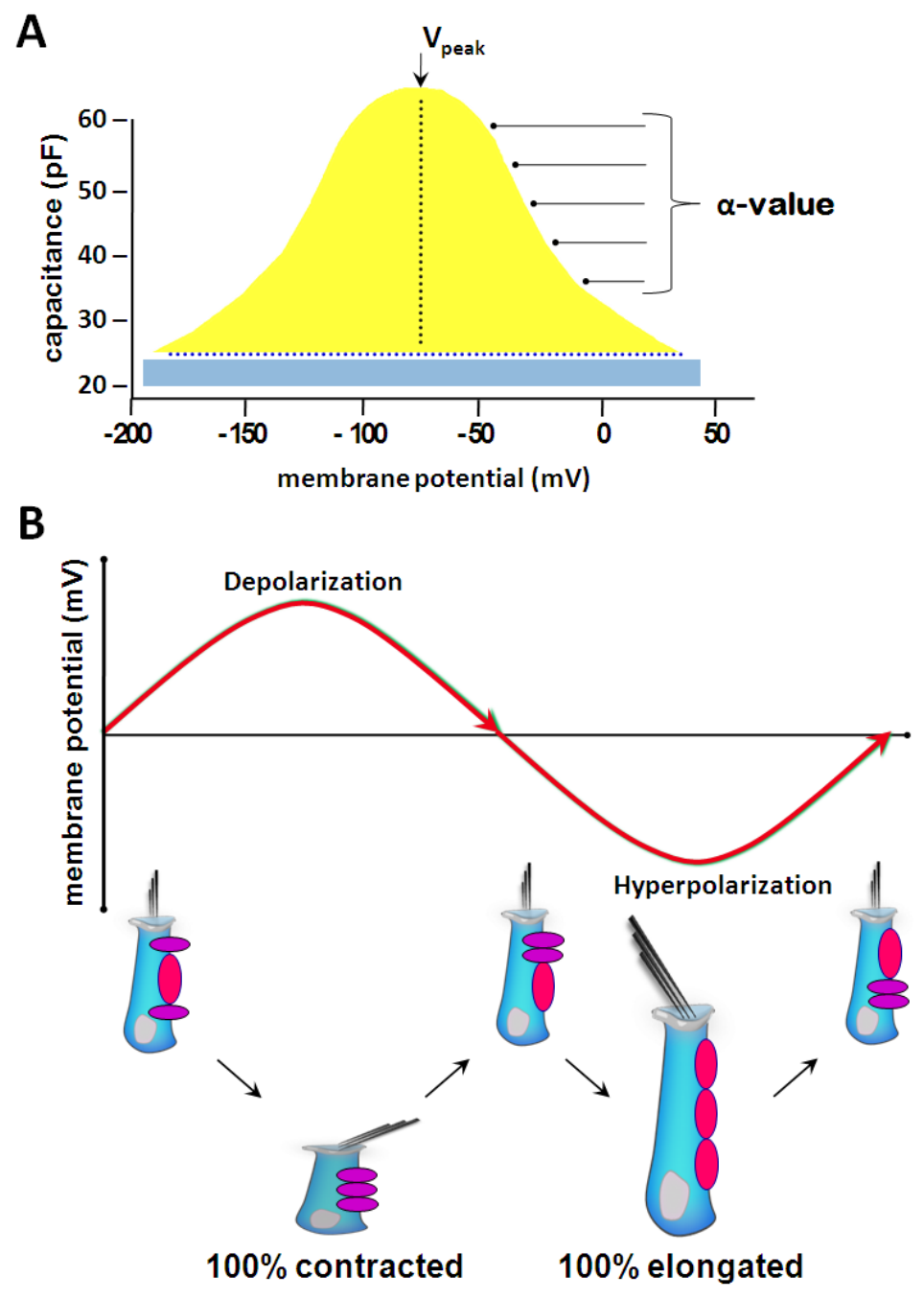

Figure 1-2 Voltage-dependence of prestin electromotility within the membrane (A) NLC derives from the membrane potential. $\mathrm{V}_{\text {peak }}$ is the voltage at the peak capacitance. Capacitance equates to the derivative of charge over voltage; thus, the curve reflects charge movement through the membrane as prestin changes conformation. As more prestin change conformational, charge movement increases producing the 'nonlinear' slope of the capacitance (yellow). The membrane's linear capacitance is subtracted from the total capacitance (blue rectangle). The $\alpha$-value is the slope factor describing the voltage dependence of charge movement. Factors that change the $\alpha$-value indicate that prestin's ability to 'sense' the voltage has been altered. (B) The relationship between voltage and capacitance is a reflection of prestin's membrane state as described by two-state Boltzmann mechanics. Voltage changes indicate the probability for a particular conformation as a particle within an energy field. Thus, maximum charge movement occurs at $\mathrm{V}_{\text {peak }}$ when a $100 \%$ of prestin molecules are in the same state. The opposite holds true for hyperpolarization. The 'bell-shape' of the NLC curve is the signature for prestin function. The elongated state is represented by the vertical, pink oval. Prestin's contracted state is represented by horizontal, purple oval. 
Hearing was evaluated using two separate tests carried out on live mice. Auditory brainstem response (ABR) measures stimulation of the auditory nerve downstream from the hair cells [46]. The data showed an elevated hearing threshold of 40-60 dB. The second test, distortion product otoacoustic emissions, detects the sound emitted from the tympanic membrane which occurs as a by-product of amplification [47]. These measurements indicated a threshold increase of 45-55 dB. The hearing tests concluded that the knockout animals were deaf. Collectively, the functional experiments showed that hearing sensitivity had decreased in a frequency-dependent manner. This signified that prestin's removal abolished the two defining features of cochlear function, frequency selectivity and amplification [48].

Two results from the prestin knockout complicated the functional interpretation. First, the knockout led to the elimination of IHCs and OHCs along 25\% of the cochlear spiral in the basal region. Secondly, the average length of the OHCs in the entire cochlea had shortened by $15 \%$ [48]. These outcomes suggested that the elevated hearing threshold might have resulted from the reduction in cell number or from structural defects in the OHCs. To address these concerns, a knockin mouse was created that impaired prestin's functional ability without inhibiting placement in the membrane. A knockin mouse was created, referred to as '499', with point mutations at residue 499 (V499G) and $501(\mathrm{Y} 501 \mathrm{H})$. These residues occur immediately after the presumed last transmembrane segment and form the N-terminal end of the cytoplasmic tail. The 499 mutation was based on earlier tests using Human Embryonic Kidney (HEK) 293T cells that were transfected with the prestin mutant. The V499G Y501H did not prevent membrane targeting; however, it significantly weakened prestin's NLC. Based on immunostaining and western blot analysis, prestin in the 499 mouse was localized to the membrane compared to the wild type. Functional characterization, using isolated OHCs, revealed a significant reduction of NLC and electromotility at the membrane potentials associated to normal OHC function. The researchers made note of the fact that the 499 mutation appeared to cause a large positive shift to the membrane potential. Measurements cannot be made at large positive potentials because they cause the cell's membrane to breakdown. In summary, these experiments overcame the limitations from the prestin knockout mouse and presented definitive evidence for prestin's motor function.

\subsubsection{Prestin Function Derives from the OHC Membrane}

Prestin's activity can be described in terms of how its behavior within an electrical field produces large-scale effects for the OHC. The OHCs membrane exhibits voltage dependent movement relative to the point of stimulation along the lateral wall. This can be observed by sending an electrical stimulus through a patch pipette that contacts different parts of the membrane [38]. This demonstrates that the motor component, which generates cellular movement, is distributed throughout the entire lateral wall of the cell. Stimulating an $\mathrm{OHC}$, constrained at the basal end in a microchamber and leaving the other end free causes the cell's extremes to move in opposite directions. By restricting movement of the cell at one end, the result shows that each motor functions independently [49]. Further experiments refined that conclusion by using dissociating the membrane activity from the rest of the cell. Trypsin, introduced 
through a patch pipette, digested the cortical lattice and underlying structures. The cytoskeleton's removal caused the OHCs to lose their elongated form and assume a spherical shape. Pieces of the cell's membrane, isolated in the patch electrode were still capable of movement upon electrical stimulation [12]. Positive voltages caused the membrane to bow shrinking its size. Negative potentials induced flattening allowing it to increase surface area [50]. These results illustrate that transmembrane voltage is the force that causes prestin to undergo conformational changes within the membrane.

The voltage dependence of prestin's activity indicates that it has some parallels to other voltage-gated channels (see Figure 1-3). Function in a gated channel is based in the mechanism that opens and closes the channels pore. Both events, opening and closing, are tied to movement of a charged 'gating particle' within the membrane. The particle behaves like a dipole. Movement occurs between the inner and outer face of the membrane. The dipole reorients based on attraction to the membrane's electrical potential as it changes. Transition between poles produces a brief, asymmetrical capacitance current also referred to as a 'gating current' [51]. This feature represents the signature for functioning of a voltage sensor in a protein. Three different features have been linked to its composition. Primarily, it consists of charged residues (Glu, Asp, Lys, Arg, or His) that are part of the voltage-gated protein's sequence. The dipole moment associated with aromatic side chain can also function as part of the voltage sensor. External ions can also serve as the voltage sensor. The ion will bind to charged residues in the protein based on the membrane potential. The binding event can produce conformational changes within the protein that shift the ion as a dipole in the membrane $[52,53]$.

Several studies have attempted to identify and characterize prestin's voltage sensor. Analysis of the sequence does not reveal the presence of motifs or residue patterns with similarity to known voltage sensor domains [43, 54]. Prestin's sequence has similarity to proteins in the SLC26 (solute carrier 26) family of anion transporter. Based on functional differences to the SLC26 proteins, mutational studies investigated different charged residues not conserved among the proteins. All the residues identified were part of the sequence found in the membrane. The charge was nullified by mutating the amino acid to glutamine or asparagines. The mutants were expressed in Chinese Hamster Ovary cells and tested for NLC. Not a single mutation had an effect on the $\alpha$-value. This led to the possibility that prestin used an external ion as the voltage sensor. Two conclusions supported a role for chloride serving as prestin's voltage sensor. First, removal of intracellular chloride eliminated NLC. This suggested that chloride could enter the membrane based on the voltage potential [53]. Secondly, voltage-clamped cells did not have a sustained current when chloride was present providing evidence that it functioned within the membrane [55]. In other words, prestin behaves as an incomplete transporter. Persistent current flow would suggest the movement of ions down a concentration gradient through a channel pore. This study provided evidence for chloride as the prestin's voltage sensor; however, it could not completely exclude the possibility of some intrinsic charge that had not been identified. At the very least, it established the importance of chloride to prestin function. A more recent study gave evidence to refute the idea suggesting that chloride could serve as the voltage sensor. The data showed that chloride's relationship to NLC was concentration dependent which supports the idea that 


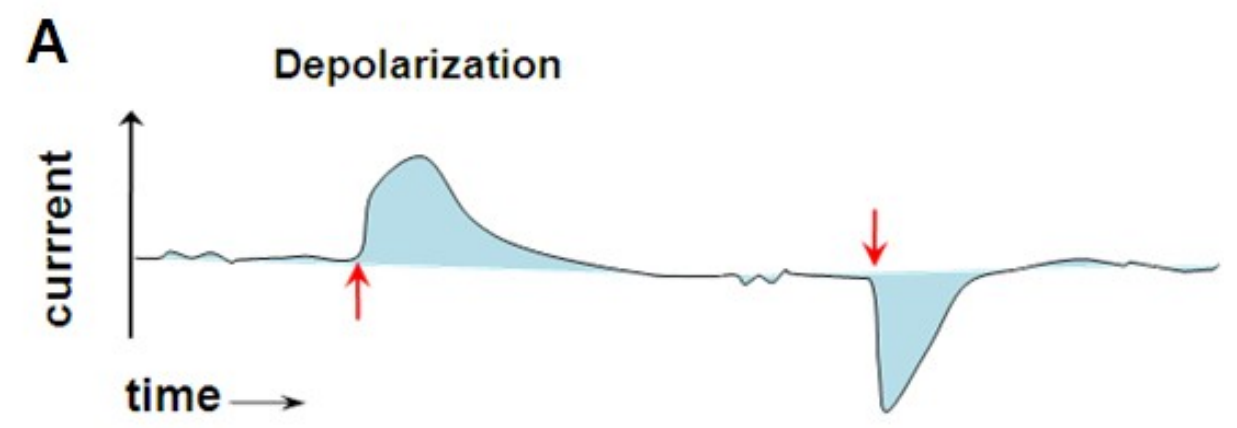

Hyperpolarization

B

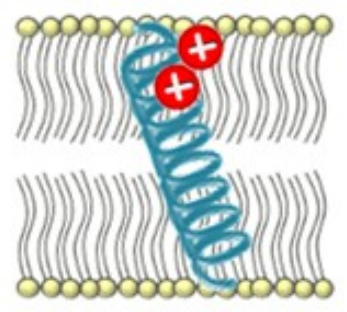

\section{Hyperpolarization}

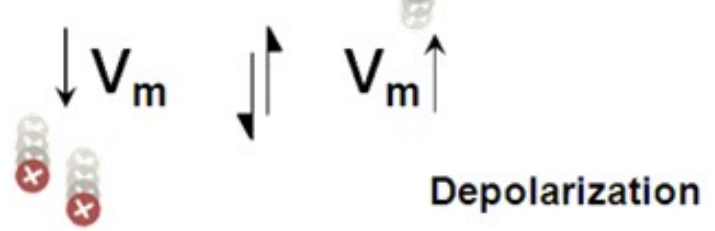

\section{Figure 1-3 Charge movement and voltage gating in prestin}

(A) Prestin exhibits a 'gating current'. Voltage-clamped OHCs will produce a brief current at the onset of depolarization. Hyperpolarization of the membrane potential will generate a second transient with reversed flow. Red arrows indicate current flow. $(B)$ Prestin has a 'gating charge'. Depolarization of the OHC shifts the membrane potential towards the positive. The electrical field pushes the gating charge towards the membrane's extracellular face. Hyperpolarization reverses the process. Presumably, some structural element within prestin's sequence, bearing charge, can readjust in response to electrical pressure. Charge movement creates the gating current, and is a feature consistent with voltage dependent conformational changes in prestin. 
it interacted with a prestin site positioned outside or adjacent to the membrane. [56].

The 'area model' of prestin function integrates features of its voltage dependence, membrane location, and conformational changes [41] to the partial transport of chloride. At hyperpolarized membrane potentials, individual prestin's expand in the membrane, which increases the surface area and cell length. Conversely, depolarized membrane potentials reduce prestin's size in the membrane. Voltage changes occur in frequencydependent cycles; thus, prestin molecules interconvert between states in synchrony to the voltage changes. On the cellular level, the sum total of prestin's state changes results in contraction and elongation of the OHCs. In conclusion, the 'area' model ties localized membrane events to movement and function for the entire cell (see Figure 1-4).

\subsection{Passive Function of Prestin and OHC Stiffness}

$\mathrm{OHC}$ function is closely tied to stiffness in the lateral wall. The tension is directed along the axis of the cell, which helps to create the cell's cylindrical shape. Two structures beneath the plasma membrane, the cortical lattice and the subsurface cisternae, form the scaffolding. The OHCs cytoplasm has a positive pressure around $130 \mathrm{~mm} \mathrm{Hg}$. The cortical lattice provides structural support beneath the membrane. The subsurface cisternae, beneath the lattice, shore up the lateral structures by encapsulating the pressurized interior [11]. Stiffness properties go hand in hand with the OHCs voltagedependence and ability to generate force. The ability to generate the force has a direct relationship to the stiffness of the OHCs lateral wall [39]. A reagent called diamide can bind to spectrin destroying the cytoskeletal structure which reduces the OHCs axial stiffness [57]. Diamide also inhibits the OHCs ability to generate force thus demonstrating the reciprocal importance of these properties to cell function [58]. The molecule salicylate, which is similar to aspirin, can affect the OHCs membrane properties in several ways. It is believed that salicylate binds near the inside surface of the plasma membrane. Salicylate has amphipathic properties, which would support close interaction with the membrane. It affects OHCs by diminishing electromotility [59], inhibiting charge movement [60], and causes significant reduction in the OHCs stiffness by disrupting contact between the membrane and underlying cortical structures [61]. Previous studies demonstrated that electromotility was a membrane function by showing that isolated pieces could bend upon electrical stimulation. The results discussed above gave a strong indication that prestin's membrane function has importance to $\mathrm{OHC}$ stiffness. However, the significance of this has been shown to extend to the resonant properties of the basilar membrane. The basilar membrane in both the prestin knockout mouse and the 499 mutant exhibited were broadly tuned. This resulted from diminished mechanical coupling between basilar membrane motion and shearing force on the stereocilia [33, 62]. In essence, these results can be considered relative to the 'area model' (see Figure 1-4). Prestin can change its size within the membrane of the OHC. In turn, these changes affect both the OHCs size and stiffness. The basilar membrane data confirms the link between prestin's conformational changes and cochlear amplification. 


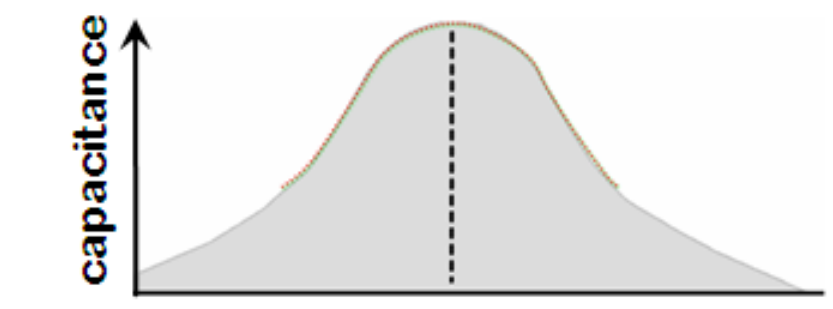

\section{$(-) m V$}
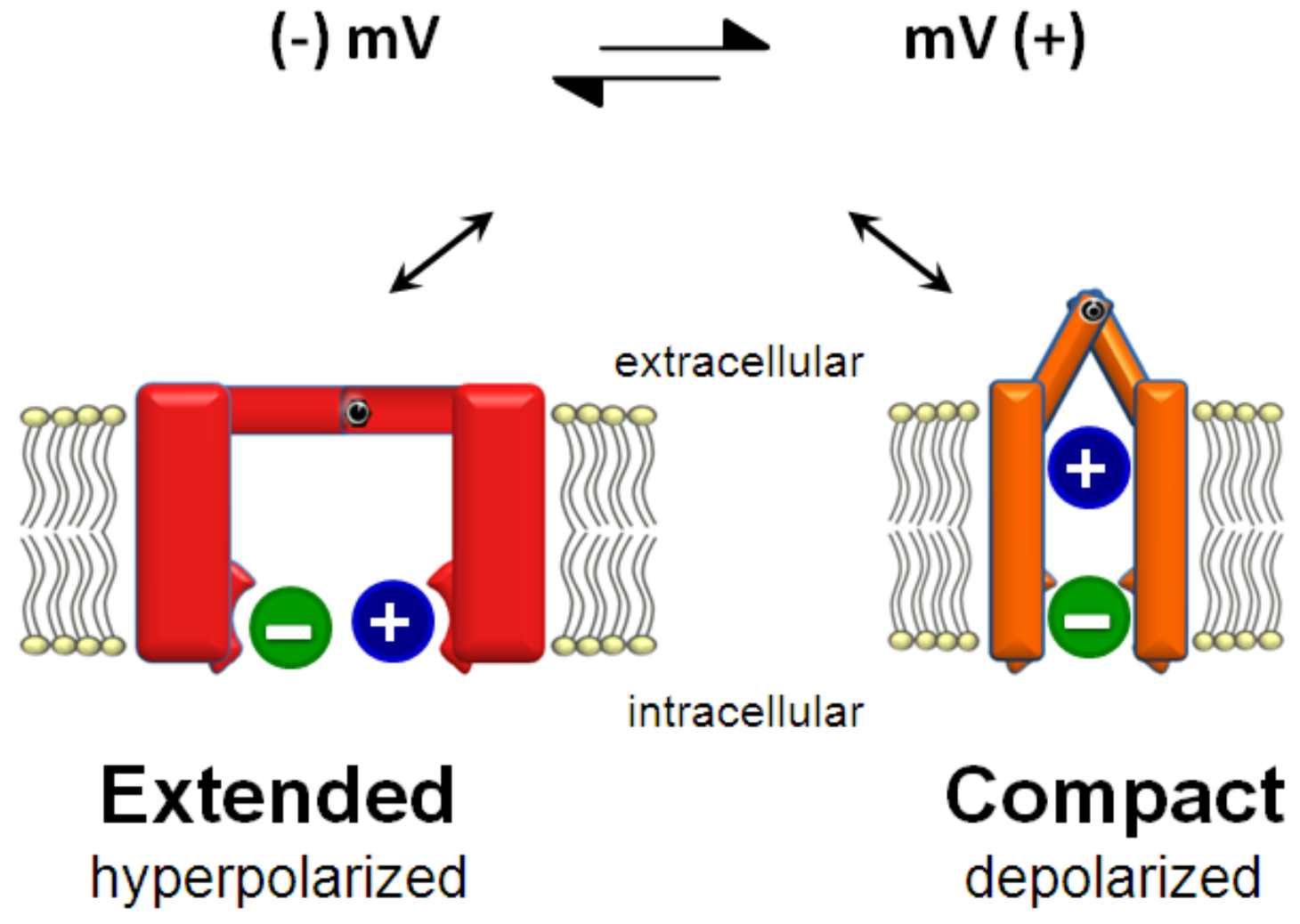

Figure 1-4 Model of prestin as a membrane-bound area motor

Chloride (green sphere) binding exerts an allosteric effect in both models of prestin motor in the membrane; however, the nature of the charge movement is the distinguishing feature between the two models. The first model (shown here) is based upon the transitioning of prestin between an extended and compact state within the plane of the membrane. The transmembrane potential determines prestin's state. Two-versions of this model exist. Charge movement originates from a charge (blue sphere), which is intrinsic to prestin within the membrane. The hyperpolarized transmembrane potential pushes chloride near the cytoplasmic face. Chloride binding induces prestin to undergo a conformational change. Prestin's charge is drawn towards the extracellular face as it becomes more negative. As the potential becomes more positive, chloride is drawn back to the cytoplasm. In the second model (not shown directly here), chloride binding produces a conformational change, while partial transport of chloride in the membrane equates to charge movement. 


\subsection{C-terminus Relationship to Prestin Function}

\subsubsection{Mechanism of Prestin-prestin Interaction Unknown}

Morphological and biochemical evidence indicated that prestin-prestin interaction occurs within the $\mathrm{OHC}$ membrane. In the $\mathrm{OHC}$, freeze-etched replicas of the lateral plasma membrane show an array of dense particles spread across the inner surface [63]. The particles are around $8-10 \mathrm{~nm}$ in diameter and positioned directly over the cortical cytoskeleton and subsurface cisternae. It has been suggested the particles may consist of multimers of prestin. The numerical density of the particles falls between 3,000-6000 $\mathrm{mm}^{2}[12,64]$. This value has reasonable agreement to numerical estimates for the number of prestin molecules. This number can be calculated from the cells' charge density. Several lines of evidences support the idea that prestin molecules are the main component of these particles. First, the strongest evidence comes from atomic force microscopy experiment of prestin transfected Chinese Hamster Ovary cells. Prestin's expression corresponded to the appearance of numerous 8- to 12-nm particles on the cell surface. Untransfected cells did not have similar structures. Additionally, fluorescence labeled antibodies to prestin produced a signal in prestin-transfected membrane preparations [65]. Another study expressed FLAG-tagged prestin from SF9 cells and then purified the protein. Native-Page analysis and size-exclusion chromatography indicated that prestin formed into tetramers [66]. In a related study, co-immunoprecipitation experiments from prestin transfected HEK $293 \mathrm{~T}$ cells show the formation of prestin dimers and tetramers [67]. Prestin proteins tagged with N-terminal or C-terminal YFP or CFP molecule displayed fluorescence resonance energy transfer or FRET signals due to their proximity in the membrane. The FRET signal suggested that prestin-prestin interaction occurred primarily through the N-terminus over a 6-10 nm distance [68].

\subsubsection{Specific Functional Role of the C-terminus Is Unclear}

NLC is a voltage-dependent activity specific to prestin's function in the membrane. Data from several studies has hinted that prestin's C-terminus is important for membrane targeting and function within the membrane. One study made a series of truncations from the end of the C-terminus starting at residue 744 and going to residue 709. These constructs were then expressed in TSA201 cells, a HEK 293 subclone. Each prestin mutant was evaluated for correct membrane targeting and for function by measuring NLC. The study showed that each larger truncation had a greater effect on membrane targeting. Consequently, NLC diminished as well. Deletions beyond residue 719 abolished membrane targeting. NLC could still be measured until truncation at residue 709 . This study demonstrated that the last 35 residues of the $\mathrm{C}$-terminus have an integral role in prestin membrane targeting and NLC [69]. It was unclear in the study whether the truncations caused specific effects relative to the NLC. It is possible that the results could be explained by structural defects caused by the deletions.

Perhaps the strongest suggestion linking the C-terminus to prestin's voltage function comes from the 499 mutant mouse. Although not proved directly, the two point 
mutations probably left the C-terminus intact without causing structural problems. This can be inferred from the truncations studies. Problems with membrane targeting were not observed. At the same time, NLC and electromotility were diminished by over $90 \%$. As a comparison, the C-terminus truncation studies indicate that the C-terminus' structure is of critical important for prestin function. Presumably, the truncations affected the folding based on the membrane targeting results. The implication from these two studies is that the C-terminus must be vital to prestin's membrane activity. The 499 mutation could have simply reoriented the $\mathrm{C}$-terminus in such a way that prevented association with the membrane.

Direct evidence comes from data showing the importance of charged residues in the C-terminus to prestin's voltage-dependence. Point mutations were introduced that reversed the charge in three areas of the $\mathrm{C}$-terminus. None of the mutations diminish the NLC [70]. However, sensitivity to the previous operational-voltage shifted to either more positive or more negative membrane potentials. The polarity of the voltage-shift depended upon the location of charge within the sequence [70]. Collectively, the results suggested that the C-terminus was not part of the voltage sensor otherwise; the mutations would have reduced charge movement. At the same time, the data supported a role tied to modulating the voltage-readiness of the entire protein, which would imply that the $\mathrm{C}$ terminus uses an allosteric mechanism to regulate prestin's energy state and voltage sensitivity.

\subsubsection{SLC26 Anion Transporters and Sulfate Transporters}

Prestin belongs to the 'Solute Carrier 26' protein family of integral anion transporters or SLC26. Eleven proteins classified in the SLC26 group show affinity for a variety of anions to include chloride, iodide, sulfate, and bicarbonate. A 22-residue consensus motif links the SLC26 proteins to the larger Sulfate Permease (SulP) family of transporters [71]. This class of proteins encompasses a broad range of organisms from prokaryotes to plants to vertebrates [43]. Both SLC26 and SulP proteins have intracellular $\mathrm{N}$ and $\mathrm{C}$-termini and a hydrophobic core of 10-14 transmembrane segments [72]. Prestin also has intracellular termini; however, it is not clear whether it has ten or twelve transmembrane segments $[68,73]$. Figure 1-5 highlights these features of prestin's sequence. The C-terminus of all proteins in SulP family contains a predicted sulfate transporter and anti-sigma (STAS) domain [74]. In both SLC26 and SulP transporters, The C-terminus in both families appears to have a consistent functional relationship to membrane anion transport [75-78]. The mechanism remains unknown and seems to differ between proteins relative to undefined sequence attributes.

The STAS domain of the sulfate transporters, Sultr1;1 and Sultr1;2, has been shown to directly influences membrane targeting and sulfate transport. This appears to be a property of the domain's structure. Point mutations targeted to either to $\beta$-sheet or $\alpha$ helix elements blocked sulfate transport and disrupted membrane trafficking. Similar results were obtained by complete deletion of the STAS in Arabidopsis thaliana Sultr1;2 [78]. Replacement of the deleted STAS with a different STAS domain restored membrane localization; however, the kinetics of sulfate transport were altered, indicative 


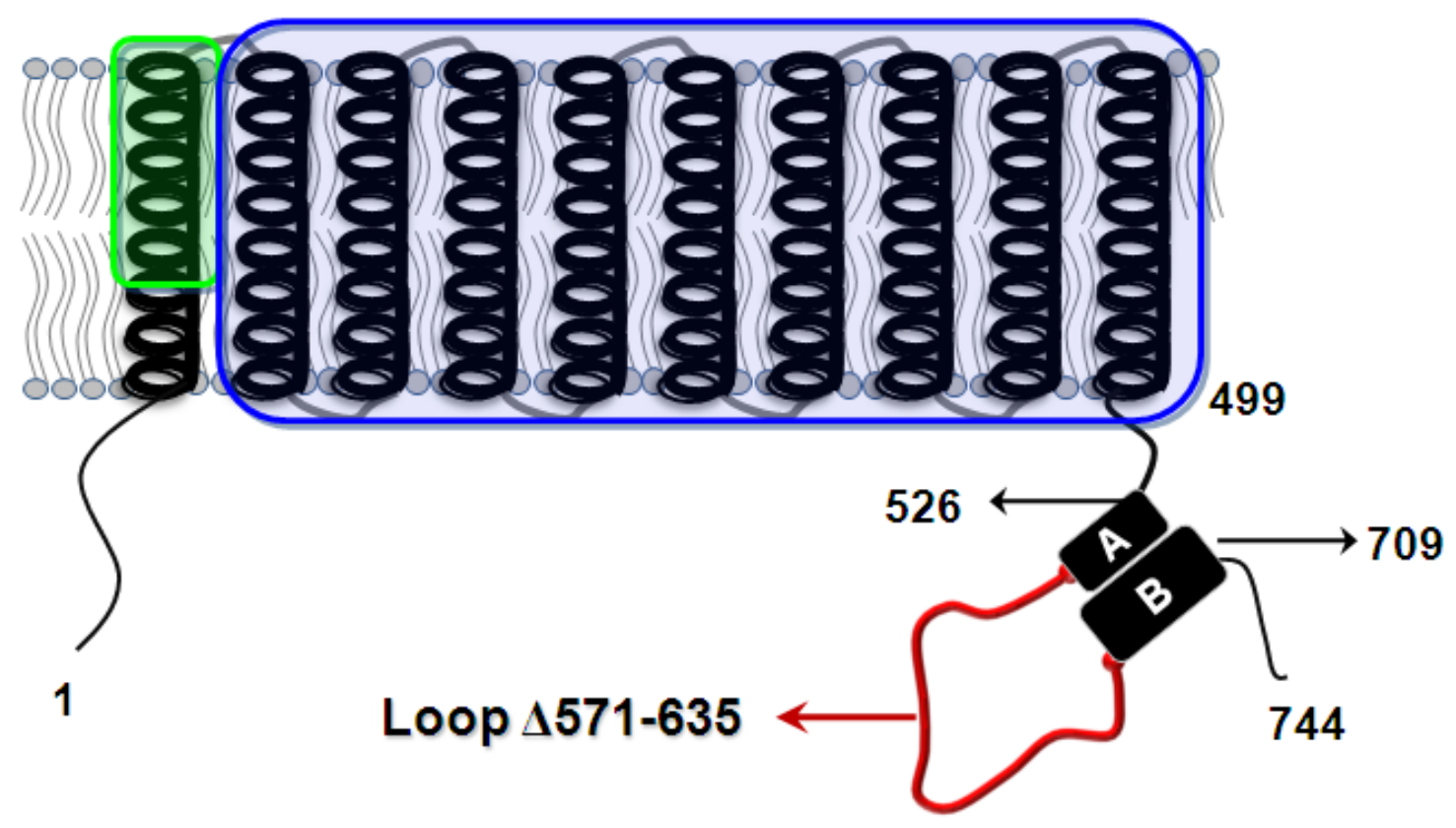

Figure 1-5 Topology and sequence features of prestin

Area highlighted in green, residues 109-130, corresponds to conserved transmembrane residues used to define the SLC26 anion transporter proteins. The area framed in blue, residues 193-503, shares homology with the larger protein family of sulfate transporters. The 'A' and ' $\mathrm{B}$ ' areas represent the predicted STAS domain with residue boundaries annotated by the arrows. The loop $\Delta 571-635$ represents the disordered region between the STAS A and B investigated in these studies. 
of its function $[79,80]$. The STAS domain in SLC26 proteins appears to have a similar function to the role in sulfate transporters. Evidence for this comes from the numerous disease-related mutations found in the STAS domain of SLC26A2-A4. All of the mutations produce transport defects, which severely disrupt the cell's ionic balance [8184]. The mutations appear to destabilize the protein's ability to fold which causes improper membrane localization. Some of the mutations only disrupt the STAS domain without upsetting membrane targeting. The transport mechanism is still intact, but severely weakened.

\subsection{C-terminal STAS Domain Evolved from the Prokaryotic SpoIIaa Protein}

The STAS domain represents the predominant structural feature of prestin's Cterminus. All proteins in the SulP family contain a predicted STAS domain $[43,74]$. The origin of the domain's name stems from shared homology with SpoIIaa found in Bacillus species. The link between the SpoIIaa protein and eukaryotic anion transporters is largely based on predictive analysis. The STAS was identified from a blast search seeded with SpoIIaa sequence. The results pulled out C-terminal sequence from SLC anion transporters. The reciprocal search referenced against transporter sequence yielded the same results. Secondary structure prediction-based threading was able to show alignment between four predicted $\beta$-stands. Nuclear Magnetic Resonance (NMR) and crystallization have produced several structures for the Bacillus proteins [85, 86]; however, a similar structure for the eukaryotic domains remains forthcoming. The SpoIlaa proteins have between 110-120 residues. The structure consists of a two-layer $\alpha-\beta$ sandwich with a terminal $\alpha$-helical handle [87-89]. A small conserved loop appears to have general NTP binding function. SpoIIaa serves as an antagonist to the SpoIIab anti-sigma factor protein. Binding of SpoIIaa prevents SpoIIab from inhibiting transcription during sporulation; thus, the domain's function is based on protein-protein interaction [88]. As such, the STAS domain may have a similar general function in eukaryotes [72]; however, the domain is often part of a larger protein suggesting alternative roles may have evolved [90].

Analysis of the STAS domain in prestin's C-terminus reveals several overt differences between prokaryotic and eukaryotic STAS domains. Sequence alignment between prestin's STAS, SpoIIaa STAS, and the C-terminal STAS from related anion transporters shows that only weak homology exists between the proteins (see Figure 1-6). The prokaryotic STAS consists of an uninterrupted, linear sequence; whereas, the alignment shows that the eukaryotic STAS domains have additional sequence inserted within the domain. The extra sequence exhibits variable length and residue bias between the proteins. Essentially, it divides the sequence into two distinct areas that have homology to the prokaryotic STAS. The similarity between the proteins derives from the conservation of a few clusters of hydrophobic residues and a strongly conversed aspartic acid at residue 653. These two STAS areas in prestin's C-terminus will be referred to as STAS A and STAS B in these studies. Additional sequence, unrelated to the SpoIIaa STAS, appears to continue after the C-terminal end of the domain in the eukaryotic proteins. 


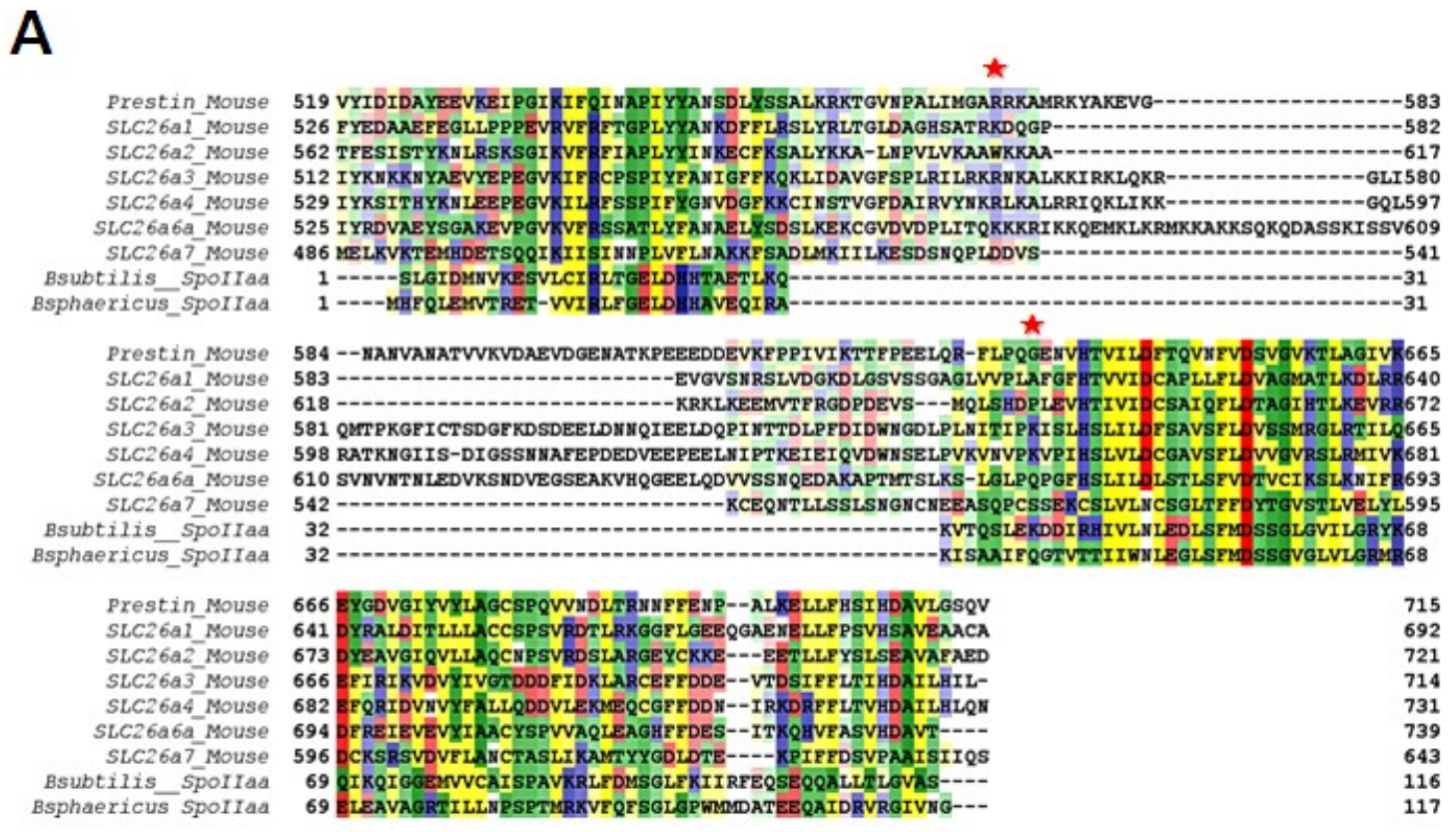

B

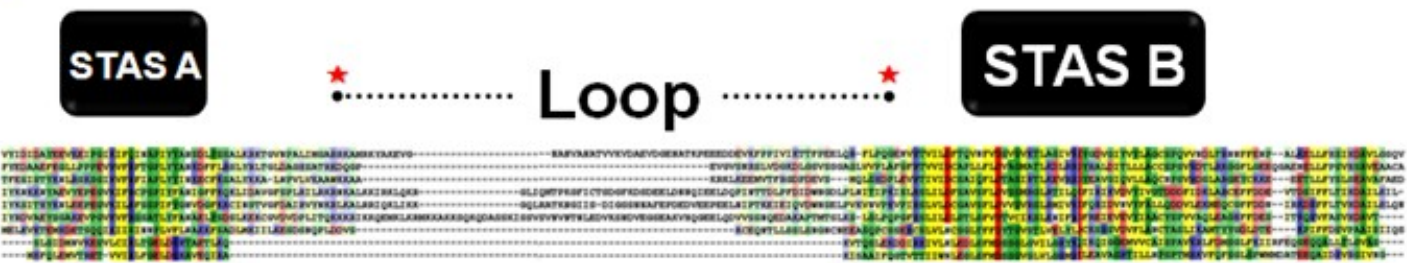

Figure 1-6 Alignment of STAS domains suggests functional differences exist (A) Sequence alignment showing weak conservation between different STAS domains. STAS B has greater similarity with SpoIIaa than the STAS A. Prestin's STAS domain is on top followed by the STAS domains of SLC26 a1-a4 and a6-a7. The last two sequence below to Bacillus SpoIIaa proteins that have had the structure solved. The two red stars mark residues 571-635, which corresponds to the disordered loop characterized in these studies. The sequences are colored according to amino acid characteristics. Yellow residues are strongly hydrophobic, red corresponds to acidic residues, blue residues are basic, and green residues are small or polar. (B) Location of STAS A, the loop, and STAS $\mathrm{B}$ relative to the alignment presented. 
Overall, the weak alignment and the insertion sequence imply structural and functional changes occurred as the STAS domain evolved in prestin's sequence [90-92]. Thus, the prokaryotic STAS may not serve as the best reference for prestin's C-terminus. The weak correlation has made it difficult to determine the domain's location within the sequence of prestin's C-terminus. In that regard, several prestin papers have been published each listing a different position for the domain [68, 69, 74]. This supports the need to characterize the structure of the $\mathrm{C}$-terminus in order to unify our understanding field.

Secondary structure prediction and disorder prediction results seem to parallel the sequence alignment (see Figure 1-7). Although the boundaries are not exact, the predicted secondary structure overlaps with the sequence aligned to the STAS domain. An area of random coil corresponds to the sequence inserted within the STAS boundaries. The first area, residues 583-639, would form a loop between the sequence predicted for the STAS domain in prestin's C-terminus. The second area, residues $717-744$, falls at the Cterminal end of the predicted STAS. Several structures of the Bacillus SpoIIaa STAS domain have been solved. Overlay of the prediction to SpoIIaa structure displays variability in where the structures could align. This suggests that the predicted structure in residues 583-639 does not have relevance. The Protein Homology/analogY Recognition Engine or Phyre program generated a consensus secondary structure prediction by combining the independent results from PSIPRED [93], SSPro [94], and JNet [95] programs. Intrinsically unstructured proteins (IUP's) or disordered protein sequence contain a bias towards specific amino acids including proline, serine, glutamine, and lysine. This produces areas of high net charge compared to hydrophobicity, which manifests as areas of 'invisibility' in crystal and NMR structures due to the lack of electron density. The PONDR method of disorder prediction takes these types of factors into consideration. Submission of full-length prestin and the C-terminus sequence to the PONDR VSL1 and VLXT algorithms yielded similar results. The PONDR VSL1 method compares the inputted sequence to short ( $<30$ residues) and long ( $>30$ residues) stretches of disorder found in proteins with known 3D structure. The PONDR VLXT integrates the VSL1 method along with two other neural networks trained on specific types of disorder to provide greater accuracy. Output above a score of 0.5 indicates disorder. The output from the disorder prediction indicates that residues 560-635 and 711-744 may lack structure. Both areas of sequence overlap with the two areas of random coil from the secondary structure results. Based on this similarity, it was hypothesized that approximately $60-70$ residues located between residues 560 and 635 are not required for the protein to fold correctly. Thus, this sequence exists as a flexible loop.

\subsection{Testable Objectives and Project Strategy}

Prestin's C-terminus and STAS domain have functional importance to the protein's voltage-dependent activity; however, a specific role has not been identified [53, 69]. Despite the functional relevance, the prestin field lacks insight concerning the structure, folding, and biochemical properties of the $\mathrm{C}$-terminus. This idea is clearly reflected in the literature discussing prestin's STAS domain. Inconsistencies, related to 
A

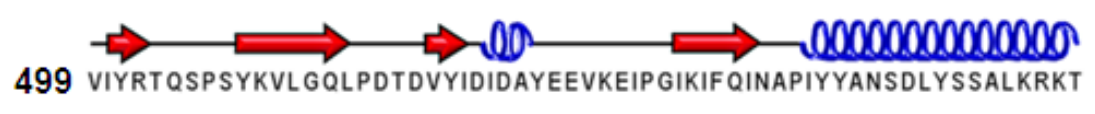

561 GVNPALIMGARRKAMRKYAKEVGNANVANATVVKVDAEVDGENATKPEEEDDEVKFPPI
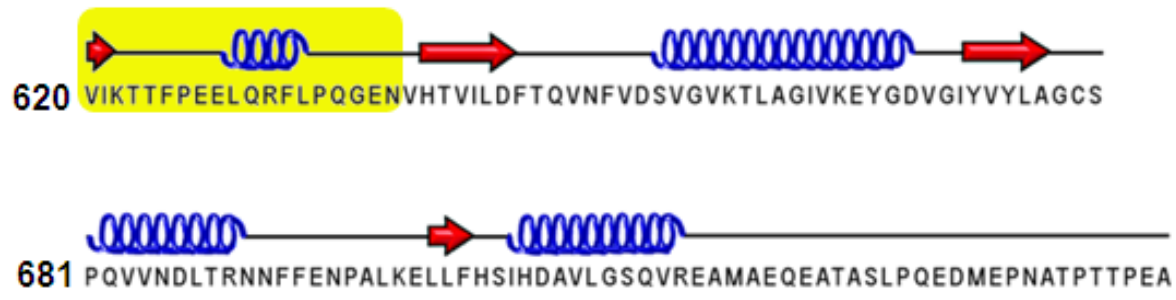

B

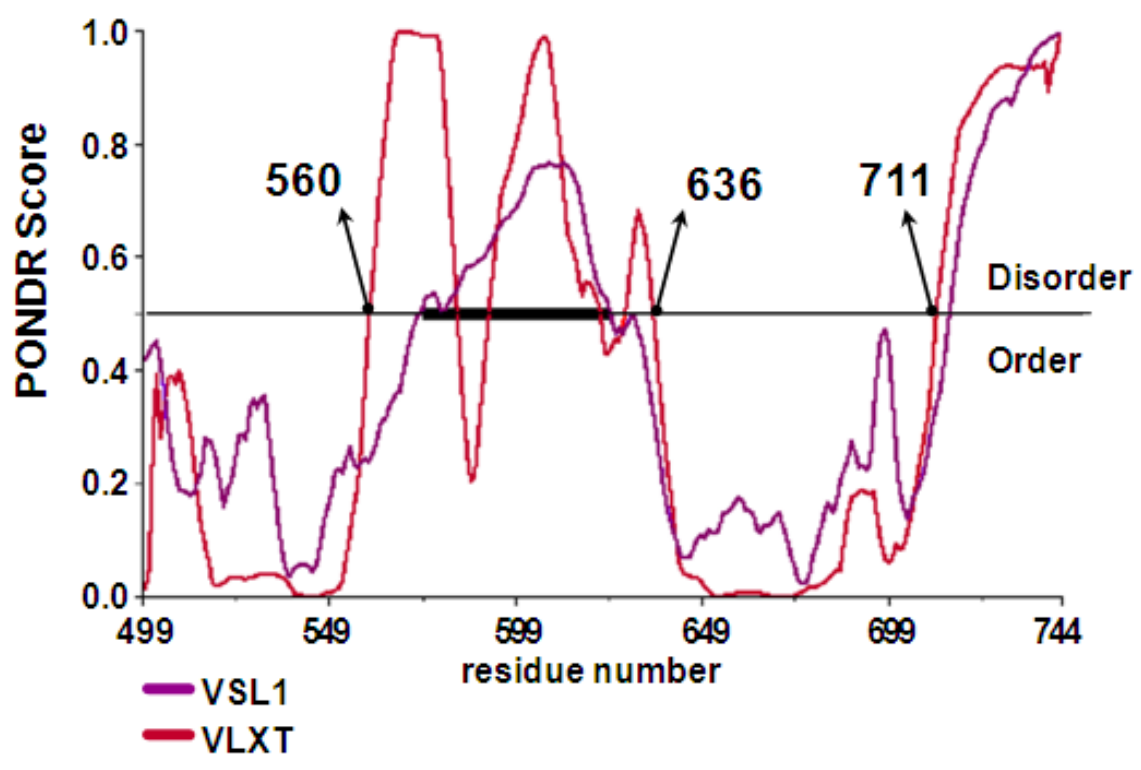

Figure 1-7 Secondary structure and disorder predictions for the C-terminus Residues 499-744 from mouse prestin were used for both predictions. (A) The secondary structure prediction represents a consensus of several different prediction methods which were generated by the Phyre protein fold program [96]. The area highlighted in yellow corresponds to residues 571-635. (B) The PONDR disorder prediction method identified two areas in the C-terminus lacking fixed structure. Two different algorithms were used. The VSL1 method (purple line) is trained to identify disordered areas shorter than 30 residues and longer than 30 residues. The VLXT (red line) is optimized to identify long regions of disorder. Values above 0.5 indicate disorder. The sequence boundaries for the predicted disorder are marked with the arrows. The black bar between 570 and 636 represents a disordered area of at least forty residues in sequence. 
the domain's location and size, are presented in several publications. Residues 634-710 [69], 584-707 [68], 528-709 [97], and 505-744 [70] are all credited to the domain. At present, the most salient information about the domain comes from the prokaryotic SpoIIaa-STAS protein, which has had several structures solved [85, 88, 98]. Prestin's STAS displays several clusters of conserved hydrophobic residues, which link it to the prokaryotic domain. Between sequences of weak homology, the residue may persist due to the structural importance as they pack into the protein's folded cores. Therefore, these amino acids serve as good marker points for the STAS domain's location.

To understand the relationship between the observed folding and the predicted STAS domain, the sequence of prestin's C-terminus was aligned to the SpoIIaa STAS and to the C-terminal STAS of related transporters. The comparison highlighted two distinct regions with weak homology to the prokaryotic STAS domain. These two areas were called 'A' (STAS A) and 'B' (STAS B). The homology to the prokaryotic STAS derives from the conservation of only a handful of hydrophobic residues and a strongly conversed aspartic acid at residue 653. The eukaryotic sequence, including prestin, has sequence inserted between the two parts of the STAS and tailing from its C-terminal end. The inserted sequence exhibited variable length and residue bias between the proteins. The alignment implies that SpoIIaa STAS may not serve as a good marker for the domain's boundaries in prestin's C-terminus. Perhaps in relation, earlier prestin studies mapped the STAS domain to different locations within the C-terminus [68, 69, 74]. These models are not consistent with the evidence presented here.

Based on the initial sequence analysis, it was hypothesized that prestin's STAS domain consists of two subdomains, which form the primary structure in the C-terminus. The remaining sequence, approximately 130 residues, likely does not contribute to a stable fold. To test these ideas, different recombinant proteins were designed, each with sequence deleted from a particular area of sequence. The deleted residues corresponded to the sequence predicted to lack structure. The solution behavior and structural properties were characterized using analytical ultracentrifugation (AUC) and NMR. The resulting data served as an outline to map out the boundaries of the STAS domain.

Finally, the relationship between prestin's voltage-dependent activity and the Cterminus' structure has not been explicitly studied. Comparison of STAS domain sequence across kingdom and phyla only shows weak homology. It also reveals the presence of a highly variable region inserted between predicted secondary structure elements, which are attributed to the STAS domain [74]. It was hypothesized that the variable region may have evolved to perform a specific function in the C-terminus. Based upon the recombinant protein studies, this sequence was deleted from the sequence of full-length prestin. The deletion mutant was expressed in eukaryotic cells to characterize its functional role in respect to membrane targeting and NLC. 


\section{Chapter 2 Materials and Methods}

\subsection{Sequence Alignment, Secondary Structure, and Disorder Prediction}

All protein sequences, including SLC26 Mus musculus proteins a1-a7, were obtained from the National Library of Medicine's Entrez protein database as part of the public domain. Sequence alignments utilized ClustalW as a service of the European Bioinformatics Institute [99]. The alignment editor, Jalview v2.4, was used to prepare the alignment for presentation. Jalview v2.4 is available from the University of Dundee [100]. Secondary structure prediction represents a consensus generated by the Protein Homology/analogY Recognition Engine or Phyre program. The Phyre server combines the independent results from PSIPRED [93], SSPro [94], and JNet [95] programs. Residues 499-744 from mouse prestin sequence were submitted to the Phyre server [96]. Sequence for full length prestin and the C-terminus sequence were submitted to the PONDR server for analysis with the VSL1 [101] and VL-XT [102] predictions. The PONDR VSL1 method compares the inputted sequence to short ( $<30$ residues) and long ( $>30$ residues) stretches of disorder found in proteins with known 3D structure. The PONDR VL-XT method searches for disordered regions at least 40 residues in length. It is based on the integration of three neural networks trained on specific types of disorder from crystallographic and NMR structure data. Output above a score of 0.5 for both methods indicates disorder. The secondary structure and disorder prediction results are located in Figure 1-7.

\subsection{Preparation of DNA Constructs for Protein Expression}

The full-length prestin cDNA was inserted into the pEYFP-N1 vector (Clontech/Invitrogen; Carlsbad, CA) using EcoR1 and SacII restriction sites (see Figure 2-1). The protein was expressed from this vector in HEK $293 \mathrm{~T}$ cells for the in vitro studies. Residues 499 to 744 from Mus musculus prestin constitute the cytosolic Cterminus as listed in the Ensembl database (European Bioinformatics Institute and Wellcome Trust Sanger Institute) [103]. The mouse prestin coding sequence for fulllength prestin and residues 499-744 was PCR amplified from a prestin cDNA clone provided by Dr. Jing Zheng. This sequence was then inserted into the pGEX-4T1 plasmid (GE Life Sciences; Uppsala, Sweden) or the pET-28a plasmid (EMD Biosciences; Darmstadt, Germany) using BamH1 (5') and Xho1 (3') restriction sites for both plasmids (see Figure 2-1). The C-terminus proteins were expressed from pGEX-4T1 excluding 512-712 $\Delta 571-635$, which was expressed from pET-28a. All C-terminus proteins, except two, had the residues GSG added to their N-terminus from the BamH1 site. Proteins 499744 and 499-744 $\Delta 601-606$, used in the subtilisin digest experiment, were cloned into pGEX-4T1 using a 5' EcoR1 site, which added GSPEFR to their N-terminus.

Point mutations or deletions were introduced to prestin cDNA using two-rounds of PCR reactions (see Figure 2-2). In the first round, two PCR reaction were carried out to introduce the mutation to separate but overlapping halves of the sequence. Each PCR 


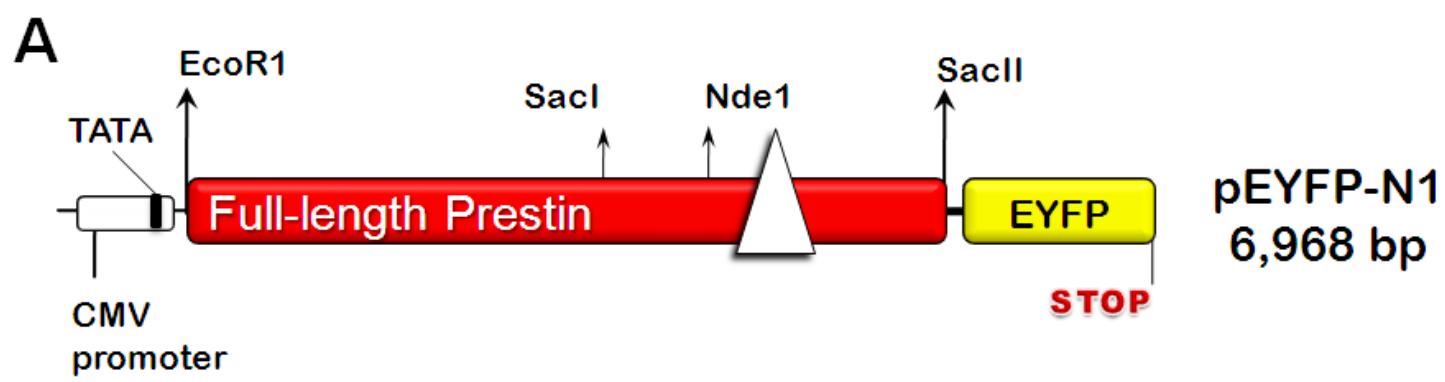

B

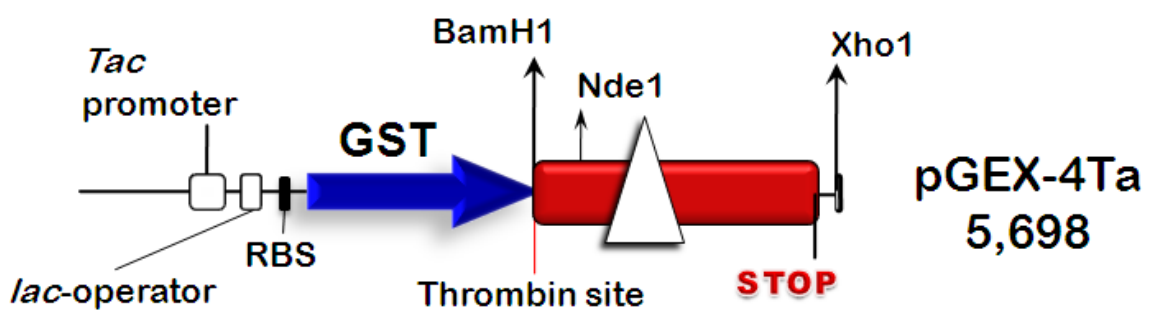

C

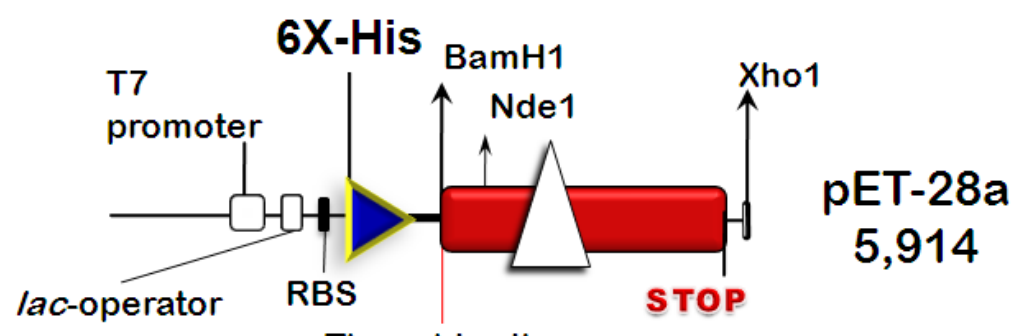

Thrombin site

Figure 2-1 Schematic of pDNA vectors and restrictions sites used in cloning (A) The pEYFP vector was used to express prestin-eYFP $\triangle 571-635$ and prestin-eYFP 1744 in HEK 293T cells. The same restriction enzyme sites were used for both proteins. (B) The pGEX-4T1 plasmid has resistance to ampicillin (not shown), which was used for selection. The insert shown corresponds to 499-744. The majority of the C-terminus proteins were expressed from this plasmid. (C) The pET-28a plasmid has resistance to kanamycin (not shown). Only the 512-712 $\Delta 571-635$ protein was expressed from this plasmid. The white triangle outlined in black indicates the relative location of residues 571-635, which were deleted from the wild type sequence. 


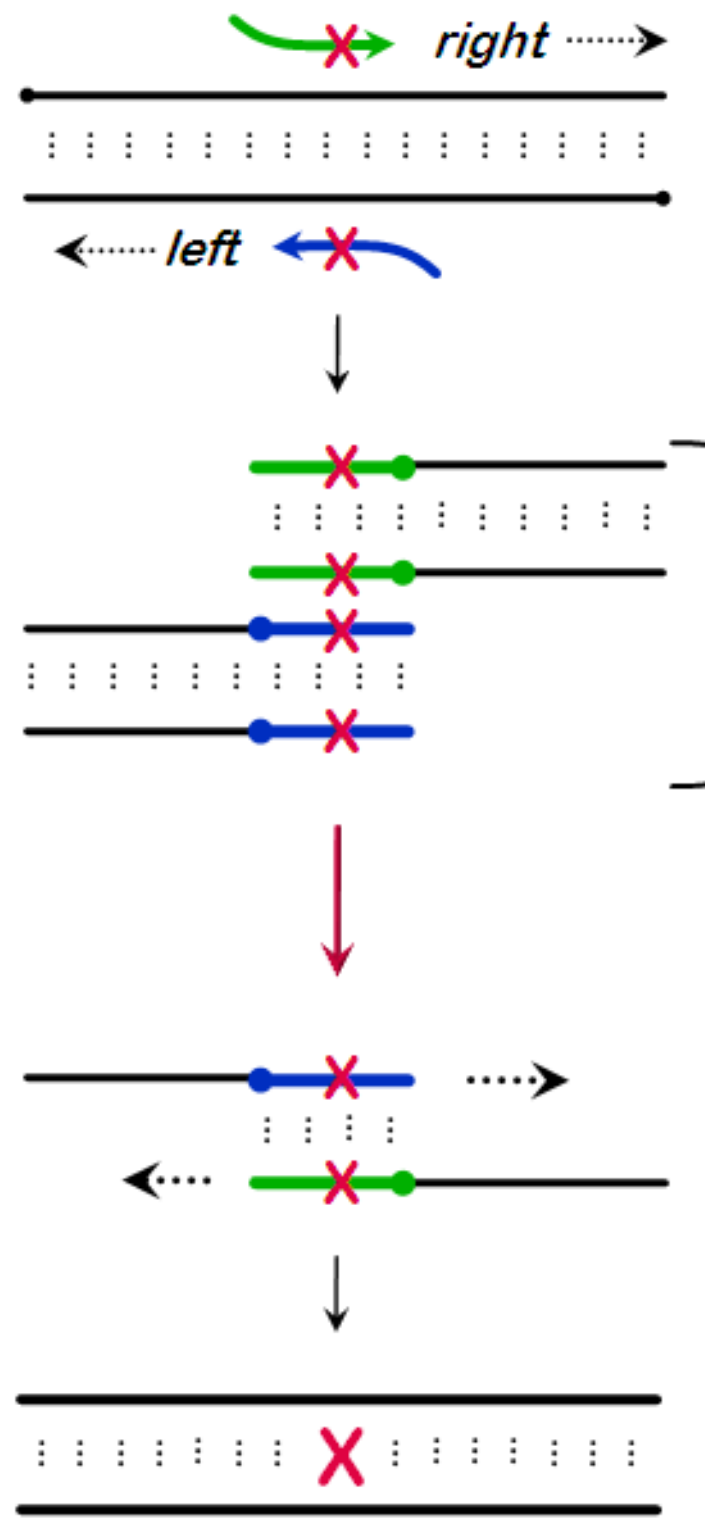

Round \#1 PCR

2 separate PCR reactions

round\#1 PCR products

\section{Round \#2 PCR}

Combine round\#1 products

Templates 'prime' each other

Figure 2-2 Two-round PCR method used to mutate prestin DNA

This method served to introduce point mutations or deletion to an area of sequence within the DNA. The red ' $\mathrm{X}$ ' represents the site targeted for the mutation or deletion. The two 'Round \#1' PCR reactions use primers (blue and green arrow) which are complementary to each other and contain the mutation. The resulting 'left' and 'right' PCR products served as templates for each other in 'Round \#2' to recreate the full DNA sequence plus mutation. Two primers were used for each PCR reaction; however, the cartoon only shows the primer containing the desired mutation. The 5' and 3' terminal oligos, which would add the restriction sites for cloning, are not shown. 
required an internal oligo containing DNA for the mutation plus DNA complementary to the template on either side of the mutation (5' and 3'). The oligos were approximately 30 to 50 bases in length which created $30-50$ bases of complementary sequence between the two PCR products. In the second round, the 'overlapping' DNA annealed together which 'primed' the polymerase to fill-in the overhanging ssDNA. The second step 'zipped' the two pieces of DNA (plus mutation) into a single piece of dsDNA. The agarose gel in Figure 2-3 depicts the two round-one PCR products that were combined to delete the 'loop' cDNA (571-635) in full-length prestin. The PCR reaction components and thermocycler settings can be found in Table 2-1 and Table 2-2, respectively. The oligonucleotides used to PCR each construct are listed in Table 2-3. Stocks for each pDNA construct were grown in $4 \mathrm{ml}$ cultures of DH5- $\alpha$ competent cells (Invitrogen; Carlsbad, CA) and purified with a mini-prep kit (Qiagen; Germantown, MD). Stocks for the pEYFP-N1 constructs were grown in $500 \mathrm{ml}$ cultures. The pDNA was purified using a maxi-prep kit (Qiagen; Germantown, MD). Automated DNA sequencing confirmed the sequence for each construct. Oligonucleotide synthesis and DNA sequencing were performed by the Hartwell Center at St. Jude Children's Research Hospital.

\subsection{Culture of HEK 293T Cells for NLC Experiments}

\subsubsection{Culture from Frozen Stock}

DMEM media was warmed at $37^{\circ} \mathrm{C}$ in preparation for the cells. Warmed media ( 9 $\mathrm{ml}$ ) was added to a $100 \mathrm{~mm} \times 10 \mathrm{~mm}$ plate and incubated at $37^{\circ} \mathrm{C}$ to maintain the plate at temperature until addition of the cells. Upon removal from liquid nitrogen, the vial of cells was thawed quickly at $37^{\circ} \mathrm{C}$. Prior to complete thaw, the outside of the cells vial was rinsed with $70 \%$ ethanol inside a sterile hood. The cells were gently resuspended using a sterile pipette tip and transferred to the plate containing warm media equilibrated to the incubator conditions. The plate was immediately returned to the incubator after the addition of the cells. After 24 hours growth, the cells were checked for attachment to the plate under a microscope. The media was replaced with fresh media to remove the DMSO used during the freezing process. The media added had been warmed to $37^{\circ} \mathrm{C}$ prior to adding it to the plate. The cells were incubated at $37^{\circ} \mathrm{C}, 5 \% \mathrm{CO}_{2}$, and $>97 \%$ humidity until $70-80 \%$ confluent in a monolayer before passage.

\subsubsection{Ethanol Precipitation of DNA for HEK 293T Transfection}

HEK 293T cells were maintained at $37^{\circ} \mathrm{C}$ in $100 \mathrm{~mm} \times 10 \mathrm{~mm}$ plates in a volume of $10 \mathrm{ml}$ of media. The incubator used to grow the cells was kept at $37^{\circ} \mathrm{C}$ with $5 \% \mathrm{CO} 2$ and $>97 \%$ humidity. The culture media consisted of Dulbecco's Modified Eagle Medium (Invitrogen, Carlsbad, CA) containing 4,500 mg/L D-glucose, L-glutamine, and 110 $\mathrm{mg} / \mathrm{L}$ sodium pyruvate. The media was supplemented with $10 \%$ fetal bovine serum (FBS). Prior to passage of the cells, media and Dulbecco's Phosphate-Buffered Saline buffer (D-PBS) (Invitrogen, Carlsbad, CA) was incubated at $37^{\circ} \mathrm{C}$ to warm them. Media was removed from the plate containing the HEK 293T cells and $10 \mathrm{ml}$ of D-PBS was 


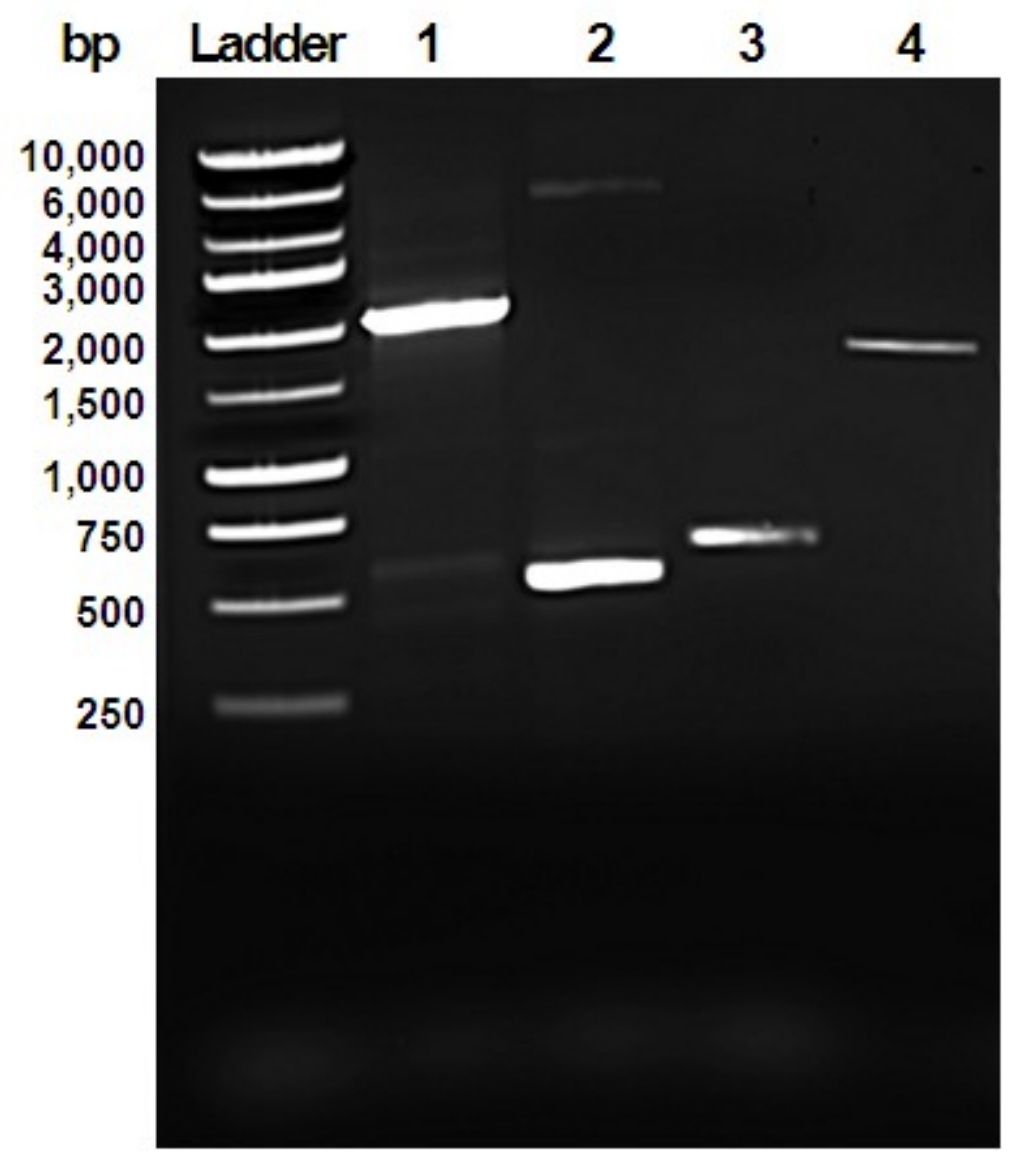

Figure 2-3 Agarose gel of the PCR products combined to delete 'loop' DNA The gel shows the DNA fragments combined to create the full length prestin $\Delta 571-635$ construct expressed in HEK 293T cells. Lanes 1 shows the PCR product from round two which is the completed full length prestin $\triangle 571-635$ dsDNA. Lanes 2 and 4 contain DNA resulting from the two PCRs in round one. The DNA in lane 2 ('right' PCR reaction) lacks the cDNA which codes for the loop. This DNA was amplified from the 499-744 $\Delta 571-635$ protein construct. Lane 4 ('left' PCR reaction) has wild type DNA located 5' (upstream) from the sequence in lane 2. The two products in lane 2 and 4 overlap. This allowed them to serve as primers for each other in the round two PCR reaction where they were combined into the final product in lane 1. Lane 3 contains PCR amplified DNA for wild type C-terminus 499-744, as a control. 
Table 2-1 PCR reaction components

\begin{tabular}{ll}
\hline Reagents & Volume $(\mathbf{5 0} \boldsymbol{\mu l}$ total $)$ \\
\hline dNTPs & $1 \mu 1$ \\
10X PFU buffer & $5 \mu 1$ \\
pDNA template & $0.6 \mu 1$ \\
5' oligo $10 \mu \mathrm{M}$ & $1 \mu \mathrm{l}$ \\
3' oligo $10 \mu \mathrm{M}$ & $1 \mu \mathrm{l}$ \\
Sterile $\mathrm{H}_{2} \mathrm{O}$ & $40.4 \mu \mathrm{l}$ \\
\hline
\end{tabular}


Table 2-2 PCR thermocycler settings

\begin{tabular}{lll}
\hline Step & Temperature & Time \\
\hline Initial denaturing step & $95^{\circ} \mathrm{C}$ & 4 minutes \\
Denaturing temperature & $95^{\circ} \mathrm{C}$ & 30 seconds \\
Annealing temperature & $49^{\circ} \mathrm{C}$ & 30 seconds \\
Extension temperature & $72^{\circ} \mathrm{C}$ & 1 minute \\
Final extension & $72^{\circ} \mathrm{C}$ & 10 minutes \\
Hold temperature & $4^{\circ} \mathrm{C}$ & $\infty$ (until terminated) \\
\hline
\end{tabular}


Table 2-3 Oligonucleotide primers used for prestin constructs

\begin{tabular}{|c|c|c|}
\hline Protein construct & DNA site & Oligonucleotide sequence \\
\hline \multirow[t]{2}{*}{$499-744$} & 5' end & cgcggatccggcgtgatttatagaacacagagtcc \\
\hline & 3 ' end & gtgggtggtgggggctccgtatcggcgagctcgcc \\
\hline \multirow[t]{4}{*}{ 526-744 polar } & 5 ' end & cgccgcggatccggaggatatgaggaggtgaaagaaattcctgg \\
\hline & left & catctgcctcttttacgatgttttggacagcagcagaacaaccag \\
\hline & right & cagcagcagaacaaccaggtcaaatttcccccaatagtcatcaaaacaac \\
\hline & 3' end & gtgggtggtgggggctccgtatcggegagctcgcc \\
\hline \multirow[t]{4}{*}{$499-744 \Delta 601-618$} & 5 ' end & cgcggatccggcgtgatttatagaacacagagtcc \\
\hline & left & cagtttcacctacgtcttcatctgtatcagtagttttgttgtaaaggac \\
\hline & right & gtcaaagtggatgcagaagtagacatagtcatcaaaacaacatttcctg \\
\hline & 3' end & gtgggtggtgggggctccgtatcggegagctcgcc \\
\hline \multirow[t]{4}{*}{$499-744 \Delta 571-635$} & 5 ' end & cgcggatccggcgtgatttatagaacacagagtcc \\
\hline & left & gggtcgtgagtaataccetcgcccacgtcgecctcccettttacaggtgtg \\
\hline & right & gcgggaggggaaaatgtccacactgtcattctagactttacgcaggtc \\
\hline & 3 ' end & gtgggtggtgggggctccgtatcggcgagctcgcc \\
\hline \multirow[t]{2}{*}{$526-744 \Delta 571-635$} & 5' end & cgccgcggatccggaggatatgaggaggtgaaagaaattcctgg \\
\hline & 3' end & gtgggtggtgggggctccgtatcggcgagctcgcc \\
\hline \multirow[t]{2}{*}{$499-709 \Delta 571-635$} & 5 ' end & cgcggatccggcgtgatttatagaacacagagtcc \\
\hline & 3 ' end & caaggtgtcataggtgctacgtatcggcgagctcgcc \\
\hline \multirow[t]{2}{*}{$512-712 \Delta 571-635$} & 5' end & cgcggatccggcgggcagctccetgacacggatgtgtac \\
\hline & 3' end & gtgtcataggtgctacgtcaggacccgatcggcgagctcgec \\
\hline \multirow[t]{2}{*}{ STAS B 613-744 } & 5' end & cgcggatccggcgaagtcaaatttcccccaatagtcatc \\
\hline & 3' end & gtgggtggtgggggctccgtatcggcgagctcgcc \\
\hline \multirow[t]{3}{*}{ Prestin $\Delta 571-635$} & 5' end & accggaattctcgccaccatggatcatgctgaagaaaatgaaatc \\
\hline & right $\Delta$ & tcctggggcagctccetgacacggatgtg \\
\hline & 3' end & atcccegcggtgcctcgggggtggtgggtgtggcattggg \\
\hline
\end{tabular}


briefly added to the plate and then removed to wash the cells. Afterwards, $1 \mathrm{ml}$ of $0.005 \%$ trypsin EDTA was added to the plate. The plate then incubated at $37^{\circ} \mathrm{C}$ for 3 minutes. After incubation, $10 \mathrm{ml}$ of warm media was added to resuspend the cells, which were then transferred to a $50 \mathrm{~mL}$ conical tub for centrifugation at $800 \mathrm{rpm}$ for 3 minutes. After completion of the spin, the supernatant aspirated, and $10 \mathrm{ml}$ of warm, fresh media was added to resuspend the cells. New plates were started using $1 \mathrm{ml}$ of the resuspended cells to $9 \mathrm{ml}$ of media.

Sterile $100 \mu 1$ stocks were made of each construct used for prestin expression in HEK 293T cells. Each stock had a $1 \mu \mathrm{g} / 1 \mu \mathrm{l}$. Filter-sterilized $5 \mathrm{M} \mathrm{NaCl}(0.2 \mu \mathrm{M}$ filter $)$ was added at $1 / 25$ the total volume of pDNA. Filter-sterilized $100 \%$ ethanol $(0.2 \mu \mathrm{M}$ filter) was added to the mixture at a ratio of two and half times the volume. This step causes the pDNA to precipitate. The pDNA was centrifuged for ten minutes at 13,000 rpm using a small bench-top Eppendorf centrifuge. Pay attention to the tube's orientation within the centrifuge. Depending upon the amount of pDNA, a pellet may or may not be visible after the spin. From this point, the pDNA is handled using sterile-technique under a culture hood. Excess ethanol was removed using sterile pipette tips being careful to avoid the bottom-outer edge of the tube. Leave tube open inside the hood for several minutes to dry any residual ethanol. Add $100 \mu 1 \mathrm{TE}$ Buffer $(0.2 \mu \mathrm{M}$ sterile-filtered $)$ to the $100 \mu \mathrm{g}$ of lyophilized pEYFP pDNA for wild type and the deletion mutant.

\subsubsection{Transfection of HEK 293T Cells}

Prior to transfections, the HEK 293T cells were grown in a $100 \mathrm{~mm} \times 10 \mathrm{~mm}$ plate. The cells were maintained at a count of $1.5 \times 10^{6}$ cells / $\mathrm{ml}$ in preparation for the transfections. The FuGENE 6 Transfection Reagent (Roche Applied Science; Upper Bavaria, Germany) was used to transfect prestin-eYFP pDNA into the HEK 293T cells. FuGENE is a lipid-blend that forms a complex with DNA and transports it into the cell. The ratio of prestin-eYFP pDNA to FuGENE reagent was based on instructions for the FuGENE product. A pipette was used to add $600 \mu 1$ of Opti-MEM (serum free) media, and $18 \mu \mathrm{l}$ of FuGene 6 to a sterile $15 \mathrm{ml}$ conical tube. The FuGENE was added carefully to avoid contact between the un-diluted reagent and the tubes plastic wall. The mixture was incubated at room temperature for 5 minutes. After which, eight $\mu$ l of prestin-eYFP stock DNA $(8 \mu \mathrm{g})$ from the ethanol precipitation was added to the mixture of FuGENE and Opti-MEM (serum free) media. The completed mix was swirled gently to mix and then incubated at room temperature for 15 minutes. This solution was added to a 100 $\mathrm{mm} \times 10 \mathrm{~mm}$ plate containing $10 \mathrm{ml}$ of warmed DMEM media and grown overnight at $37^{\circ} \mathrm{C}$ in the incubator. The following day, the cells were passaged in preparation for electrophysiology experiments. The health of the cells was checked under the microscope before passage. The procedure followed did not vary from the steps outlined for normal cell passage except for the plate. After the centrifugation, the cells were resuspended in $10 \mathrm{ml}$ of fresh media. $100 \mu$ of fresh media was added to center of a $50 \mathrm{~mm}$ MaTEK glass bottom plate. The dish has a $14 \mathrm{~mm}$ square glass fixed to the bottom of the dish. The glass has been treated with poly-d-lysine, which help the cell to adhere prior the experiments. A volume of 10-25 $\mu \mathrm{l}$ of cells was dropped into media already in the dish. The plates were incubated at $37^{\circ} \mathrm{C}$ for $2-3$ hours before electrophysiology experiments. 
Prestin expression was determined based on the fluorescence observed from the eYFP tag after stimulating the cells with a wavelength of $512 \mathrm{~nm}$. The cells were analyzed with a Nikon LiveScan SFC (swept field confocal) microscope equipped with a 63X oil immersion, 1.4 numerical aperture objective. Images were scanned from the upper to lower edges at $0.8 \mathrm{~mm}$ intervals using an EZ-C1 detection system.

\subsection{Electrophysiology Methods}

Electrophysiology experiments recorded cells using the whole-cell voltage patchclamp configuration at the room temperature. Approximately 21 cells were patched in total from three independent transfections. Pipettes were pulled with a pipette puller (Model P-2000, Sutter Instruments; Novato, CA). The resistance of the patch pipettes used was between 2 and $3 \mathrm{M} \Omega$ when filled with an intracellular medium (140 mM CsCl, $2 \mathrm{mM} \mathrm{CaCl}_{2}, 5 \mathrm{mM}$ EGTA and $10 \mathrm{mM}$ HEPES). In order to facilitate to measure capacitance, channel-blocking ions were also used in external medium. The external solution contained $140 \mathrm{mM} \mathrm{NaCl}, 5 \mathrm{mM} \mathrm{CsCl}, 2 \mathrm{mM} \mathrm{MgCl}, 1 \mathrm{mM} \mathrm{CaCl}, 2 \mathrm{mM} \mathrm{CoCl} 2$ and $10 \mathrm{mM}$ HEPES. The osmolarity of each medium was adjusted to $300 \pm 5 \mathrm{mOsm} / \mathrm{kg}$ with glucose. The $\mathrm{pH}$ was adjusted to 7.4. A patch amplifier (Axopatch 200B, Axon Instruments; Union City, CA) was used for patch clamp experiments. Cells were patched at holding potential $0 \mathrm{mV}$. Nonlinear capacitance was measured by using a windowsbased whole-cell voltage clamp program jClamp (SciSoft, CT USA). In order to obtain NLC, the patched cell was applied with a continuous high resolution two-sine stimulus protocol $(10 \mathrm{mV}$ peak at both 390.6 and $781.2 \mathrm{~Hz})$ with a voltage ramp from -150 to $+120 \mathrm{mV}$ after whole-cell configuration was established. Data analysis and curve fitting was used by the software Igor Pro 6.0 (WaveMetrics, Lake Owego, OR). The NLC C $(V)$ data were fitted by a derivative of the two-state Boltzmann function that involves linear capacitance $\mathrm{C}_{\text {lin }}$, the maximum nonlinear charge transfer $\mathrm{Q}_{\max }$, the voltage at peak capacitance $\mathrm{V}_{\text {peak }}$, and the $\alpha$ slope factor:

$$
\begin{aligned}
C_{(V)} & =C_{\operatorname{lin}}+\mathrm{Q}_{\max } \propto \frac{B}{(1+B)^{2}} \\
B & =\exp \left(\propto\left(V-V_{1 / 2}\right)\right)
\end{aligned}
$$

The $\alpha$-value describes the voltage dependence of charge transfer. Data analysis and curve fitting was used by the software Igor Pro 6.0 (WaveMetrics, Lake Owego, OR).

\subsection{Bacterial Cell Transformation with pDNA}

This procedure describes the protocol to transform competent bacterial cells with pDNA. The same steps apply to DH5 $\alpha$ (Invitrogen, Carlsbad, CA) and Rosetta 2 (DE3) pLysS cells (EMD Biosciences, Darmstadt, Germany) competent cells. DH5 $\alpha$ cells were used for cloning purposes. Rosetta 2 (DE3) pLysS cells were used for protein expression Competent cells were removed from storage at $-80^{\circ} \mathrm{C}$ and thawed on ice. The cells were used immediately after thawing to avoid loss of competency. Approximately $0.5 \mu \mathrm{g}$ of 
pDNA was added to $25 \mu \mathrm{L}$ of DH5 $\alpha$ or Rosetta 2 (DE3) pLysS cells, mixed gently, and incubated on ice for 20 minutes. The tube containing the cells was heat shocked for 45-50 seconds in a $42^{\circ} \mathrm{C}$ water bath and then placed on ice for two minutes. Around $600 \mu \mathrm{L}$ of lysogeny broth [104] Miller (LB-Miller) media (EMD Biosciences, Darmstadt, Germany) was added to the cells. The cells were grown at $37^{\circ} \mathrm{C}$ for one hour with shaking at 200 $225 \mathrm{rpm}$. After an hour of growth, the cells were centrifuged for one minute at 6,000 rpm. The cell pellet was resuspended with $150 \mu \mathrm{L}$ of fresh LB-Miller media and added to a sterile LB-Miller agar plate containing the appropriate antibiotics relative to the plasmid. DH5 $\alpha$ cells were grown on a plate containing ampicillin $(150 \mu \mathrm{g} / \mathrm{ml})$. Rosetta 2 (DE3) pLysS cells were grown on a plate with ampicillin $(150 \mu \mathrm{g} / \mathrm{ml})$ and chloramphenicol (30 $\mu \mathrm{g} / \mathrm{ml})$. The freshly inoculated plates were grown overnight at $37^{\circ} \mathrm{C}$.

\subsection{Expression and Purification of Recombinant C-terminus Proteins}

The buffers and solutions used during the purification are also listed in Appendix $\mathrm{B}$, except where noted. The C-terminal recombinant proteins were expressed in Rosetta 2 (DE3) pLysS bacterial cells (EMD Biosciences, Darmstadt, Germany). Bacterial cells were grown in LB-Miller media for normal expression. Bacterial cells, which expressed

${ }^{15} \mathrm{~N}$-labeled protein, were cultured in minimal media containing ${ }^{15} \mathrm{~N}$ ammonium chloride as the only nitrogen source. The different reagents used to make the minimal media are listed in Table 2-4. Minimal media required the addition of 10X MOPS solution, 2,000X vitamin solution, and 100X micro-nutrients solution. These solutions were mixed and filter-sterilized separately from the media prior to use. Their components are listed in Table 2-5. Approximately 10-15 $\mathrm{ml}$ of an overnight starter culture was used to inoculate each one liter of LB-Miller media. The starter cultures used for ${ }^{15} \mathrm{~N}$-labeled protein expression contained minimal media instead of LB-Miller media. Around $50 \mathrm{ml}$ of an overnight starter culture was used to inoculate each liter of minimal media. A single colony of transformed bacterial cells was used to start each overnight culture regardless of the type of expression. The culture was grown overnight for 16 hours at $37^{\circ} \mathrm{C}$ while shaken at $200 \mathrm{rpm}$. The appropriate antibiotic(s) was added to the media prior to the inoculation. Each antibiotic was diluted 1,000 fold relative to the culture's volume.

One day before expression, sterile LB-Miller or minimal media was prepared in 2.5 liter culture flasks. Each flask was warmed to $37^{\circ} \mathrm{C}$ to ensure uniform growth. The appropriate antibiotic(s) were added as stated previously. Each liter culture was inoculated with $10-15 \mathrm{ml}$ of the starter culture and grown at $37^{\circ} \mathrm{C}$ while shaken at 200 rpm. After 2 hours, the optical density of each culture was checked using the spectrophotometer. Upon reaching a density of 0.6-0.7 absorbance units, $1 \mathrm{ml}$ of $0.6 \mathrm{M}$ IPTG was added to each liter culture to induce expression of the target protein. Immediately after, the shaker's temperature was dropped to $16^{\circ} \mathrm{C}$. Shaking at $200 \mathrm{rpm}$ continued for at least 16 hours until harvesting of the cells.

After overnight expression, each 1 liter culture was transferred to a 1 liter centrifuge bottle and spun at 4,500 pm for 10-15 minutes to pellet the cells. The mediasupernatant was disposed after completion of the spin. Each liter pellet was resuspended 
Table 2-4 Minimal media components

\begin{tabular}{ll}
\hline Reagent & Volume (1 liter) \\
\hline $10 \mathrm{X} \mathrm{MOPS} \mathrm{solution}$ & $100 \mathrm{ml}$ \\
$1 \mathrm{M} \mathrm{K}_{2} \mathrm{HPO}_{4}$ & $1.32 \mathrm{ml}$ \\
$40 \%$ glucose & $10 \mathrm{ml}$ \\
$10 \%$ solution ${ }^{15} \mathrm{NH}_{4} \mathrm{Cl}$ & $10 \mathrm{ml}$ \\
$2,000 \mathrm{X}$ vitamin solution & $50 \mu \mathrm{l}$ \\
$100 \mathrm{X}$ micro-nutrients solution & $20 \mu \mathrm{l}$ \\
Sterile $\mathrm{H}_{2} \mathrm{O}$ & $879 \mathrm{ml}$ \\
\hline
\end{tabular}


Table 2-5 Solutions added to minimal media

\begin{tabular}{|c|c|c|}
\hline Solution & Reagent & Amount \\
\hline \multirow[t]{8}{*}{ 10X MOPS solution } & $1 \mathrm{M}$ MOPS pH 7.4 & $400 \mathrm{ml}$ \\
\hline & $1 \mathrm{M}$ tricine $\mathrm{pH} 7.4$ & $40 \mathrm{ml}$ \\
\hline & $10 \mathrm{mM} \mathrm{FeSO} \cdot 7 \mathrm{H}_{2} \mathrm{O}$ & $10 \mathrm{ml}$ \\
\hline & $0.25 \mathrm{M} \mathrm{K}_{2} \mathrm{SO} 4$ & $11 \mathrm{ml}$ \\
\hline & $1 \mathrm{M} \mathrm{CaCl}_{2}$ & $5 \mu \mathrm{l}$ \\
\hline & $1 \mathrm{M} \mathrm{MgCl}_{2} \cdot 6 \mathrm{H} 2 \mathrm{O}$ & $5.28 \mathrm{ml}$ \\
\hline & $4 \mathrm{M} \mathrm{NaCl}$ & $125 \mathrm{ml}$ \\
\hline & Sterile $\mathrm{H}_{2} \mathrm{O}$ & $409 \mathrm{ml}$ \\
\hline \multirow[t]{6}{*}{ 2,000X Vitamin solution } & Thiamine & $67.4 \mathrm{mg}$ \\
\hline & Pantothenic acid & $47.6 \mathrm{mg}$ \\
\hline & p-hydroxybenzoic acid & $32.0 \mathrm{mg}$ \\
\hline & p-aminobenzoic acid & $27.4 \mathrm{mg}$ \\
\hline & 2,3-dihydroxybenzoic acid & $30.8 \mathrm{mg}$ \\
\hline & Sterile $\mathrm{H}_{2} \mathrm{O}$ & $10 \mathrm{ml}$ \\
\hline \multirow[t]{7}{*}{ 100X Micro-nutrients solution } & $\mathrm{Na}_{2} \mathrm{MoO}_{4} \cdot 2 \mathrm{H}_{2} \mathrm{O}$ & $0.436 \mathrm{~g}$ \\
\hline & $\mathrm{H}_{3} \mathrm{BO}_{3}$ & $0.618 \mathrm{~g}$ \\
\hline & $\mathrm{CoCl}_{2}$ & $0.178 \mathrm{~g}$ \\
\hline & $\mathrm{CuSO}_{4}$ & $0.043 \mathrm{~g}$ \\
\hline & $\mathrm{MnCl}_{2}$ & $0.396 \mathrm{~g}$ \\
\hline & $\mathrm{ZnSO}_{4}$ & $0.072 \mathrm{~g}$ \\
\hline & Sterile $\mathrm{H}_{2} \mathrm{O}$ & $500 \mathrm{ml}$ \\
\hline
\end{tabular}


in approximately 6-12 $\mathrm{ml}$ of chilled buffer. Two different buffers were used depending upon the type of affinity tag attached to the protein. GST-tagged protein was resuspended in $50 \mathrm{mM}$ Tris $\mathrm{pH} 7.4$ and $200 \mathrm{mM} \mathrm{NaCl}$ buffer. Protein with a six histidine affinity tag (6x-His) was resuspended in $20 \mathrm{mM}$ Tris $8.0,10 \mathrm{mM}$ imidazole, and $500 \mathrm{mM} \mathrm{NaCl}$ buffer. The resuspended bacterial cells were kept on ice from this point forward. The cells were then transferred to $50 \mathrm{ml}$ conical tubes for lyses by sonication. Based on the cell's volume in the tube, $1 \mathrm{M}$ DTT was added to a final concentration of $5 \mathrm{mM}$ to the GST-tagged protein. The serine protease inhibitor, phenylmethylsulphonyl fluoride (PMSF) was added to a final concentration of $2.5 \mathrm{mM}$. The cells were sonicated while kept on ice. The sonicator was set at a constant pulse and an intensity of 50 percent. Each tube was sonicated eight seconds for six complete rounds. Tubes were placed back on ice after each eight second round. Upon completion, the lysate was centrifuged twice at high speed to remove the insoluble cell fraction. The sample was transferred to Oakridge tubes and spun for 20-minutes with $17,000 \mathrm{rpm}$ at $4{ }^{\circ} \mathrm{C}$. Clean Oakridge tubes were used between spins. A small portion of the insoluble fraction was collected using a pipette tip to run as a lane on a $10 \%$ Bis-Tris gel. Prior to the gel, this sample was resuspended in $300 \mu \mathrm{l}$ of $50 \mathrm{mM}$ Tris $\mathrm{pH} 7.4$, and then centrifuged at 13,000 rpm. This step was carried out three times to wash the sample.

The purification scheme followed three steps: affinity chromatography, thrombin cleavage to remove the affinity tag, and passage over an anion exchange column. A gel filtration column served as a fourth step for polishing dependent upon the protein's purity as determined by SDS-Page analysis. The protein's purity was assessed by running samples from each purification step onto a $10 \%$ Bis-Tris gel, which was stained with Coomassie Brilliant Blue R250. The purification of 512-712 $\Delta 571-635$ is shown in Figure 2-4. Proteins were concentrated for experiments using an Amicon stirred cell (Millipore; Billerica, Massachusetts).

Either Ni-NTA agarose resin (Qiagen; Germantown, MD, USA) or glutathione sepharose 4 fast flow resin (GE Life Sciences; Uppsala, Sweden) was used (gravity column) for the affinity purification step. The glutathione sepharose 4 fast flow resin was equilibrated with $50 \mathrm{mM}$ Tris pH 7.4, $200 \mathrm{mM} \mathrm{NaCl}$, and $5 \mathrm{mM}$ DTT buffer. The NiNTA agarose resin was equilibrated in $20 \mathrm{mM}$ Tris $8.0,10 \mathrm{mM}$ imidazole, and $500 \mathrm{mM}$ $\mathrm{NaCl}$. The amount of resin used was based on the estimated amount of protein expressed per liter (3-15 mg/liter). Ni-NTA has a $5-10 \mathrm{mg} / \mathrm{ml}$ binding capacity. Glutathione sepharose has an average binding capacity of $10 \mathrm{mg} / \mathrm{ml}$. After equilibration, the clarified supernatant was added to the column at a flow rate of $0.8 \mathrm{ml} / \mathrm{min}$. The GST column was washed with $50 \mathrm{mM}$ Tris $\mathrm{pH} 7.4,200 \mathrm{mM} \mathrm{NaCl}$, and $5 \mathrm{mM}$ DTT buffer. The Ni-NTA column was washed with $20 \mathrm{mM}$ Tris $8.0,10 \mathrm{mM}$ imidazole, and $500 \mathrm{mM} \mathrm{NaCl}$ buffer. GST-tagged protein was eluted using $50 \mathrm{mM}$ Tris $\mathrm{pH} 8.0,200 \mathrm{mM} \mathrm{NaCl}, 5 \mathrm{mM}$ DTT, and $10 \mathrm{mM} \mathrm{L}$-glutathione (reduced) buffer. Protein with a $6 \mathrm{x}$-His tag was eluted with 20 $\mathrm{mM}$ Tris $\mathrm{pH}$ 8.0, $500 \mathrm{mM}$ imidazole, and $500 \mathrm{mM} \mathrm{NaCl}$ buffer. Gel samples were prepared for each purification step and ran onto a 10\% Bis-Tris gel to verify the protein's solubility, expression level, and identify the peak fractions.

Purified thrombin enzyme (Sigma-Aldrich, St. Louis, Missouri) was used to 
A

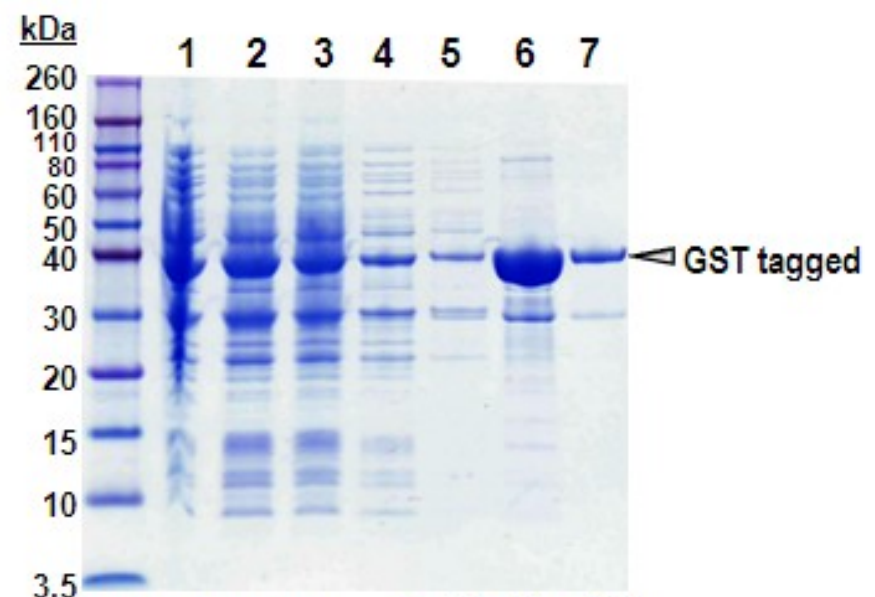

1. Whole cell 5. Elution \#1

2. Supernatant 6 . Elution \#2

3. Flow through 7. Elution \#3

4. Wash

C

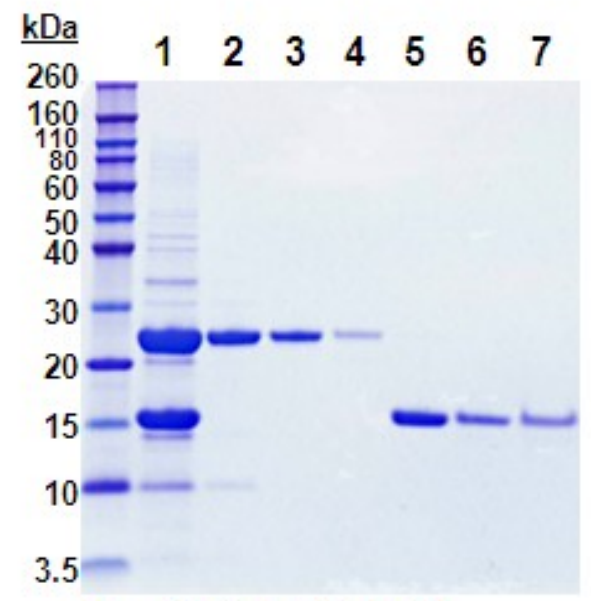

1. Q column load

2-4. Free GST

5-7. 512-712 $\Delta 571-635$
B

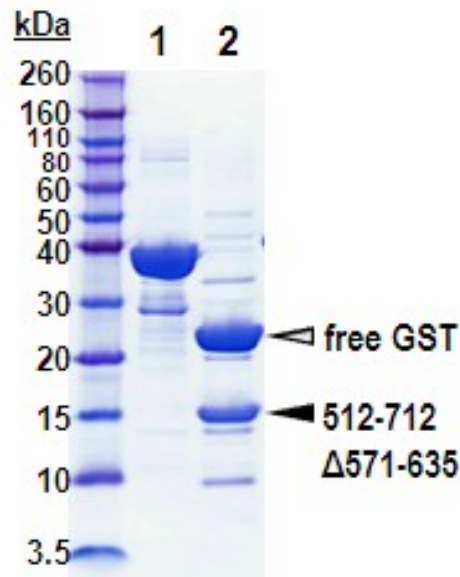

1. GST tagged

2. Thrombin cleaved

D

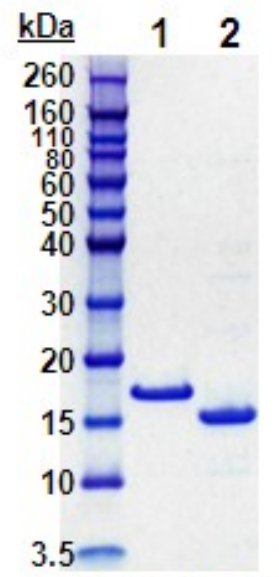

1. $6 \mathrm{x}$-His tagged

2. Cleaved

512-712 $\Delta 571-635$

Figure 2-4 Protein gels showing purification of 512-712 $\Delta$ 571-635

(A) Expression and affinity purification of GST tagged protein. (B) Removal of GST tag with thrombin. $(C)$ Q-sepharose (anion exchange) purification step. (D) Removal of 6xHis affinity tag with thrombin. 
cleave-off the affinity tag. Both the pGEX-4T1 and pET-28a plasmids added a thrombin cleavage site between the affinity tag and the start of the $\mathrm{C}$-terminus protein. Based on SDS-Page analysis, the peak fractions were pooled. The protein's absorbance was measured directly with a spectrophotometer and the concentration determined using the Beer-Lambert law. Based on the total protein, two units of thrombin were added per mg of protein along with calcium chloride to a final concentration of $2.5 \mathrm{mM}$. These samples were kept in the cold room and rotated at $15 \mathrm{rpm}$ for 16 hours. The following day, the efficiency of the cleavage was checked on a $10 \%$ Bis-Tris gel.

Anion exchange purification used occurred over a column pre-packed with Q Sepharose High Performance resin (GE Life Sciences; Uppsala, Sweden) or using a Mono Q 4.6/100 PE column (GE Life Sciences; Uppsala, Sweden) attached to an ÄKTA FPLC system (GE Life Sciences; Uppsala, Sweden). The protein was dialyzed overnight into $50 \mathrm{mM}$ Tris $\mathrm{pH} 7.4,50 \mathrm{mM} \mathrm{NaCl}$, and $5 \mathrm{mM}$ DTT buffer using a 6-8 $\mathrm{kDa}$ molecular weight (MW) cut-off D-tube dialyzer (EMD Biosciences; Darmstadt, Germany) and concentrated to $5-8 \mathrm{mg} / \mathrm{ml}$ in a volume of $10-50 \mathrm{ml}$. The $\mathrm{Q}$ sepharose or Mono $\mathrm{Q}$ column were equilibrated with $50 \mathrm{mM}$ Tris $\mathrm{pH} 7.4,50 \mathrm{mM} \mathrm{NaCl}$, and $5 \mathrm{mM}$ DTT buffer. The protein was eluted over a $0.05-1 \mathrm{M} \mathrm{NaCl}$ gradient generated with $50 \mathrm{mM}$ Tris $\mathrm{pH} 7.4,1$ $\mathrm{M} \mathrm{NaCl}$, and $5 \mathrm{mM}$ DTT buffer. The third purification step, if needed, used a highresolution 24 mL Superdex 200 10/300 GL analytical gel filtration column (GE Life Sciences; Uppsala, Sweden) as a polishing step. The protein was concentrated to 10-20 $\mathrm{mg} / \mathrm{ml}$ in a volume of 200-500 $\mu \mathrm{l}$. The protein was loaded onto the column that had been equilibrated with $25 \mathrm{mM}$ potassium phosphate $\mathrm{pH} 7.0,150 \mathrm{mM} \mathrm{NaCl}$, and $5 \mathrm{mM}$ DTT buffer. Elution occurred under a constant flow of buffer. The identity of each protein was confirmed by comparing the predicted mass to the intact mass obtained by mass spectrometry. The results for three of the recombinant proteins are shown as an example in Figure 2-5. The predicted extinction coefficient, MW, and isoelectric point for all Cterminus proteins are listed in Table 2-6.

\subsection{Proteolysis Methods}

The proteins used in these experiments were purified as described in the previous section. The buffers and solution used in the proteolysis experiments are also listed in Appendix B. Each protein was concentrated to $1.5-2.5 \mathrm{mg} / \mathrm{ml}$ using an Ultrafree $0.5 \mathrm{ml}$ Biomax-10 kDa MW cut-off centrifuge concentrator (Millipore; Billerica, Massachusetts). The samples were spun at 6,000-8,000 rcf in a micro-centrifuge for approximately five minutes. Buffer, consisting of $50 \mathrm{mM}$ Tris $\mathrm{pH} 7.6,150 \mathrm{mM} \mathrm{NaCl}$, and $5 \mathrm{mM}$ DTT, was added to each protein to dilute them to a final concentration of $1 \mathrm{mg} / \mathrm{ml}$. The same buffer was also used to hydrate lyophilized trypsin. All reactions occurred on ice for a maximum duration of 60 minutes. Separate reactions were performed for time points at two minutes, 20 minutes, 40 minutes, and 60 minutes. A fifth sample served as a control as it did not have trypsin added. Purified 499-744 protein or 499-744 $\Delta 601-618$ protein were digested using trypsin (Roche Applied Sciences; Upper Bavaria, Germany) at a ratio of 50:1. This equated to $0.5 \mu \mathrm{g}$ trypsin per $25 \mu \mathrm{g}$ protein. Each reaction was 

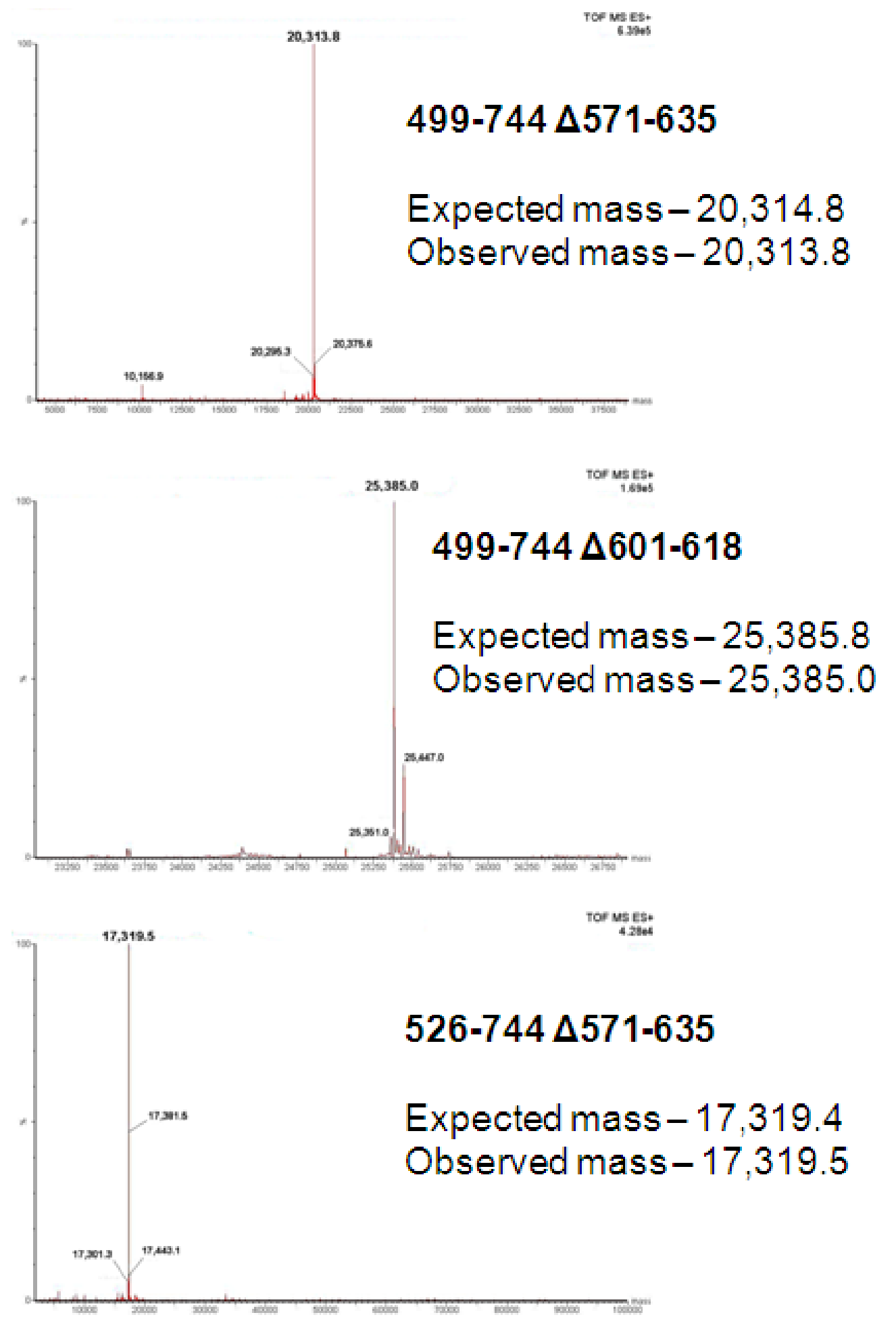

Figure 2-5 Intact mass used to confirm the identity of purified proteins

Similar intact mass results were obtained for all purified proteins used in these studies. 
Table 2-6 Predicted bio-physical features of C-terminus proteins

\begin{tabular}{llll}
\hline Construct & Extinction coefficient & MW (kDa) & Isoelectric point \\
\hline $499-744^{a}$ & 16,390 & $27,871.4$ & 4.7 \\
$499-744$ & 16,390 & $27,398.9$ & 4.7 \\
$499-744 \Delta 601-606^{a}$ & 16,390 & $27,270.8$ & 4.7 \\
$526-744$ polar & 11,920 & $24,397.6$ & 5.2 \\
$526-744 \Delta 571-635$ & 10,430 & $17,319.4$ & 4.6 \\
$499-744 \Delta 601-618$ & 16,390 & $25,385.8$ & 4.9 \\
$499-744 \Delta 571-635$ & 14,900 & $20,314.8$ & 4.5 \\
$499-709 \Delta 571-635$ & 14,900 & $16,689.9$ & 4.9 \\
$512-712 \Delta 571-635$ & 11,920 & $15,366.4$ & 4.6 \\
STAS B 613-744 & 4,470 & $14,775.6$ & 4.5 \\
\hline
\end{tabular}

${ }^{a}$ Proteins have larger MW due to the addition of six extra residues (GSPEFR) to the Nterminus during cloning. 
terminated at the time point indicated by adding 4X LDS gel-loading dye. Subsequently, the trypsin reactions were ran onto a $10 \%$ Bis-Tris gel. After completion, the gel was rinsed with milli-Q water and then soaked in 1X CAPS buffer for five minutes. At the same time, a PVDF (polyvinylidene fluoride) membrane (Millipore; Billerica, Massachusetts) was washed in $100 \%$ methanol for about 1 minute, rinsed with milli-Q water, and then placed in electroblotting buffer until needed. After soaking the gel in $1 \mathrm{X}$ CAPS buffer, the sandwich was assembled as follows: anode sponge (bottom), filter paper (wet with buffer prior to use), PVDF membrane, 10\% Bis-Tris gel, filter paper, and finally the cathode sponge on top. The gel was then transferred for one hour and ten minutes at $120 \mathrm{~mA}$ constant current at $4^{\circ} \mathrm{C}$. The electroblotting buffer was chilled to $4^{\circ} \mathrm{C}$ before use. Upon completion, the PVDF membrane was rinsed with milli-Q water for ten minutes and then placed in PVDF staining solution until bands were visible. Afterwards, the membrane was placed in destain solution for five minutes to remove the background stain. The membrane was left to air dry on a clean sheet of filter paper. The bands of interest were excised using a clean razor blade and sent to the W.M. Keck Foundation Biotechnology Resource Laboratory at Yale University. N-terminal Edman sequencing was performed according to their established protocols.

Subtilisin digest experiments also occurred in $50 \mathrm{mM}$ Tris $\mathrm{pH} 7.6,150 \mathrm{mM} \mathrm{NaCl}$, and $5 \mathrm{mM}$ DTT buffer. In this study, three reactions were carried out on the 499-744 and 499-744 $\Delta 601-606$ proteins. Each reaction tube contained $25 \mu \mathrm{g}$ of each protein in $25 \mu \mathrm{l}$ of buffer. As a control, a fourth tube did not have protease added. Subtilisin (SigmaAldrich, St. Louis, Missouri) was added in a ratio of $0.1 \%, 0.5 \%$, or $1 \%$ relative to the 25 $\mu \mathrm{g}$ of $\mathrm{C}$-terminus protein in the other three tubes. Each digest reaction transpired on ice for 60 minutes. All reactions were terminated by the addition of $5 \mu 1$ of $5 \%$ formic acid to the cap of the reaction tube followed by a pulse spin in a micro-centrifuge. The peptide fragments, which resulted from the digest, were identified by determination of their intact mass using mass spectroscopy. First, each sample was desalted using a reverse phase C4 or C8 Zip Tip column (Millipore, Billerica, Massachusetts). The proteins were eluted using a solution of $50 \%$ acetonitrile and $2 \%$ formic acid. Subsequently, the eluant was ionized by static nanospray using an EconoTip (New Objective; Woburn, MA) on a Micromass LCT mass spectrometer using positive mode. The resultant charge envelope was deconvoluted using MaxEnt 1 algorithm of MassLynx V $4.0 \mathrm{sp} 4$ software. A mass error of $1 \mathrm{Da}$ for every 10,000 Da was permissible with the mass spectrometer used.

\subsection{Analytical Ultracentrifugation Methods}

Experiments were carried out in a ProteomeLab XL-I analytical ultracentrifuge with an eight hole Beckman An-50 Ti rotor and cells containing either sapphire or quartz windows and charcoal-filled Epon double-sector centerpieces (Beckman Coulter, Fullerton, CA). For the sedimentation velocity (SV) experiments, the C-terminus proteins were dialyzed against $25 \mathrm{mM}$ potassium phosphate $\mathrm{pH} 7.0,150 \mathrm{mM} \mathrm{NaCl}$, and $1 \mathrm{mM}$ DTT buffer using a 6-8 kDa MW cut-off D-tube dialyzer (EMD Biosciences; Darmstadt, Germany). The dialysate was used as an optical reference. The buffers used in the AUC experiments are also listed in Appendix B. The loading volume of approximately $400 \mu \mathrm{l}$ 
was identical for the reference and sample chambers of the double-sector centerpiece, and the final loading protein concentrations were between 0.16 and $5.4 \mathrm{mg} / \mathrm{ml}$. The density and viscosity of the ultracentrifugation buffer was calculated from the buffer's composition and partial specific volume at $20^{\circ} \mathrm{C}$ using the public domain software, SEDNTERP, which is available from Hayes, Laue, and Philois. The MW of each protein was calculated based on the respective amino acid composition and the buffer parameters using SEDNTERP. Stokes radii and low resolution hydrodynamic shapes, using the equivalent prolate ellipsoid model, were also calculated with SEDNTERP. Following an appropriate time at rest for temperature equilibration at $20^{\circ} \mathrm{C}$, the rotor was accelerated to $60,000 \mathrm{rpm}$ and fringe displacement data were collected at time intervals of $1.0 \mathrm{~min}$ with the Rayleigh interference system for 10 hours and analyzed with SEDFIT software using the model for continuous sedimentation coefficient distribution $\mathrm{c}(\mathrm{s})$ with deconvolution of diffusional effects [105].

The sedimentation coefficient distribution $\mathrm{c}(\mathrm{s})$ was calculated with maximum entropy regularization at a confidence level of $\mathrm{p}=0.7$ and at a resolution of sedimentation coefficients of $n=100$ to 120 . The positions of the meniscus and bottom, as well as timeinvariant and radial noises, were fitted. A two-dimensional size-shape distribution, c(s, $\mathrm{f} / \mathrm{f}_{0}$ ) (with one dimension being the $\mathrm{s}$ distribution and the other the $\mathrm{f} / \mathrm{f}_{0}$ distribution) was also calculated with an equidistant $\mathrm{f} / \mathrm{f}_{0}$-grid of 0.1 steps that varied from 1.0 to 2.8 , a linear s-grid from 0.5 to $15 \mathrm{~S}$ and Tikhonov-Phillips regularization [106]. The distributions were transformed to $\mathrm{c}(\mathrm{s}, \mathrm{M})$ and $\mathrm{c}\left(\mathrm{s}, \mathrm{f} / \mathrm{f}_{0}\right)$ distributions with $\mathrm{M}(\mathrm{MW}), \mathrm{f} / \mathrm{f}_{0}$ (frictional ratio) and $\mathrm{s}$ (sedimentation coefficient) plotted as contour plots. This analysis was done with regularization. The dotted lines of the $\mathrm{c}(\mathrm{s}, \mathrm{M})$ distribution plots indicate lines of constant $\mathrm{f} / \mathrm{f}_{0}$. The distributions were not normalized [106].

Sedimentation equilibrium (SE) experiments were also performed on the 499-744 $\Delta 571-635$ and 499-744 proteins. Prior to each experiment, the proteins were dialyzed into buffer containing $25 \mathrm{mM}$ potassium phosphate $\mathrm{pH} 7.0$ and $150 \mathrm{mM} \mathrm{NaCl}$. SE was attained over $24 \mathrm{hr}$ at a rotor temperature of $4^{\circ} \mathrm{C}$ at increasing speeds of $20,000,30,000$, and 40,000 rpm for 499-744 $\Delta 571-635$ and 20,000, 30,000, and 37,000 rpm for the 499744 sample. Protein $(120 \mu \mathrm{L})$ at concentrations of $18-64 \mu \mathrm{M}$ for $499-744 \Delta 571-635$ and $10-38 \mu \mathrm{M}$ for 499-744 was loaded into double-sector centerpieces and absorbance distributions were recorded at $280 \mathrm{~nm}$ in $0.001 \mathrm{~cm}$ radial intervals with 20 replicates for each point. Global least squares modeling was performed at multiple rotor speeds with

the software SEDPHAT [107] using a single monomeric species model for 499-744 $\Delta 571$ 635 and a two discrete species model for 499-744 [108].

\subsection{Size-exclusion Multi-angle Laser Light Scattering Methods}

Size-exclusion multi-angle light scattering (SEC-MALS) experiments were carried out with a combination of an isocratic pump/auto sampler (Agilent Technologies), Shodex PROTEIN KW-802.5 (exclusion limit 60,000 Da) size-exclusion column (Showa Denko, Kanagawa, Japan) and a DAWN HELEOS static light scattering detector and an Optilab Rex refractive index detector (Wyatt Technologies, Santa Barbara, CA, USA). 
The DAWN HELEOS uses a laser wavelength of $658 \mathrm{~nm}$ and has a calibration constant of $2.2320 \times 10^{-4} \cdot 1 /(\mathrm{V} \mathrm{cm})$. The column was equilibrated with $25 \mathrm{mM}$ potassium phosphate $\mathrm{pH} 7.0,150 \mathrm{mM} \mathrm{NaCl}$, and $1 \mathrm{~mm}$ DTT buffer (see Appendix B). Experiments were conducted at $25^{\circ} \mathrm{C}$. A volume of $50 \mu$ of sample $(0.15 \mathrm{mg} / \mathrm{ml})$ in buffer was placed on the column with the auto-sampler at a flow rate of $0.3 \mathrm{ml} / \mathrm{min}$. Protein in the eluant was detected and measured via light scattering and refractive index, and the data were recorded and analyzed with Wyatt Astra software (version 5.3.2). The Zimm model was used in the analysis with the refractive index increment, $\mathrm{dn} / \mathrm{dc}$, for all proteins assumed to be $0.185 \mathrm{ml} / \mathrm{g}$ [109]. A size-exclusion profile was plotted with the laser light scattering and differential refractive index data.

\subsection{Nuclear Magnetic Resonance Spectroscopy and Circular Dichroism Methods}

Proteins used for NMR studies were isotopically-labeled $\left({ }^{15} \mathrm{~N}\right)[114,115]$ during expression from bacteria grown in MOPS-based minimal media. The only nitrogen source in the media came from the added ${ }^{15} \mathrm{~N}$-ammonium chloride [116]. Following purification, proteins were exchanged into $25 \mathrm{mM}$ potassium phosphate $\mathrm{pH} 7.0$ plus 25 $\mathrm{mM} \mathrm{NaCl}$ buffer. The protein was concentrated to $600-800 \mu \mathrm{M}$ in a volume of $300-500$ $\mu 1$ with an Amicon Ultra-15 filter unit (Millipore, Billerica, Massachusetts). Deuterated water $\left(\mathrm{D}_{2} \mathrm{O}\right)$ was added to $10 \%$ of the final volume (see Appendix B). $2 \mathrm{D}{ }^{1} \mathrm{H}^{-15} \mathrm{~N}$ TROSY spectra were recorded at $37^{\circ} \mathrm{C}$ using a Bruker Avance $800 \mathrm{MHz}$ spectrometer equipped with a cryogenically cooled TCI probe. NMRPipe software [117] processed the data collected.

Circular dichroism experiments carried out in the following buffer: $25 \mathrm{mM}$ potassium phosphate $\mathrm{pH} 6.6$ and $5 \mathrm{mM} \mathrm{NaCl}$ buffer. The protein sample and buffer were filtered using a $0.2 \mu \mathrm{M}$ membrane. The effective protein concentration for each experiment was 10-30 $\mu \mathrm{M}$. Wavelength experiments used a $300 \mu 1$ quartz suprasil cuvette with a $0.1 \mathrm{~cm}$ light path (Hellma $\mathrm{GmbH}$ and Co; Müllheim, Germany). Four scans were carried out per experiment. Each scan covered wavelengths from 195 to $260 \mathrm{~nm}$ at $1 \mathrm{~nm}$ intervals with the data averaged over 4 seconds. Thermal denaturation experiments used a $3.5 \mathrm{ml}$ quartz suprasil cuvette with a $1 \mathrm{~cm}$ light path (Hellma $\mathrm{GmbH}$ and Co; Müllheim, Germany) and magnetic stir bar. The protein was heated from a starting temperature of $15^{\circ} \mathrm{C}$ to $95^{\circ} \mathrm{C}$. The temperature was raised in $1{ }^{\circ} \mathrm{C}$ intervals. The sample was allowed to equilibrate for 1 minute at each temperature before recording. Measurements were averaged over 10 seconds for data point. Both wavelength and denaturation experiments used an AVIV model 62A DS circular dichroism spectrometer (Lakewood, New Jersey, USA).

\subsection{Surface Plasmon Resonance and Yeast Two-Hybrid Methods}

Kinetic studies of STAS A and B were performed at $25^{\circ} \mathrm{C}$ using a BIACORE 3000 (GE Life Sciences; Uppsala, Sweden) surface plasmon resonance (SPR) instrument. NeutrAvidin (Pierce) was covalently attached to a carboxymethyl dextran-coated gold 
surface (CM5 Chip) (GE Life Sciences; Uppsala, Sweden). The carboxymethyl groups of dextran were activated with $\mathrm{N}$-ethyl-N'-(3-dimethylaminopropyl) carbodiimide (EDC) and N-hydroxysuccinimide (NHS), and NeutrAvidin was attached at pH 5.0 in $10 \mathrm{mM}$ sodium acetate. Any remaining reactive sites were blocked by reaction with ethanolamine. Biotinylated STAS A peptide was then captured by injection over the chip surface. The STAS A peptides were synthesized at the Hartwell Center for Bioinformatics and Biotechnology as part of St. Jude Children's Research Hospital. The binding of 613-744 to STAS A peptide was monitored at a flow rate of $50 \mathrm{ml} / \mathrm{min}$. The STAS B 613-744 protein was dialyzed for 16 hours into $10 \mathrm{mM}$ Tris $\mathrm{pH} 7.4,150 \mathrm{mM}$ $\mathrm{NaCl}$, and $5 \mathrm{mM}$ DTT buffer using a 6-8 kDa MW cut-off D-tube dialyzer (EMD Biosciences; Darmstadt, Germany). For the experiments testing different concentrations of $\mathrm{NaCl}$, each STAS B 613-744 sample was dialyzed into buffer containing the appropriate $\mathrm{NaCl}$ concentration. SPR measurements occurred in buffer consisting of 10 $\mathrm{mM}$ Tris $\mathrm{pH} 7.4,150 \mathrm{mM} \mathrm{NaCl}, 5 \mathrm{mM}$ DTT, and 0.005\% Tween 20 (see Appendix B). Binding was measured for concentrations from 1.9 to $150 \mu \mathrm{M}$. To account for injection artifacts, a series of sensorgrams was recorded throughout the experiment after injecting only buffer (blank injections). The 613-744 dissociated completely from the chip surface, eliminating the need for a regeneration step. Data reported are the difference in SPR signal between the flow cell containing the STAS A peptide and a reference cell without the STAS A peptide. Additional instrumental contributions to the signal were removed by subtraction of the average signal of the blank injections from the reference-subtracted signal. Triplicate injections were made, and the data were analyzed globally by kinetic analysis and by equilibrium affinity analysis using the data analysis program Scrubber 2 (Version 2.0b, BioLogic Software).

Yeast two-hybrid studies were performed using the MATCHMAKER TwoHybrid System 3 (Clutch Laboratories; Palo Alto, CA). The following vectors were utilized for the respective inserts listed: pGADT7-prestin A residues 499-562; pGBKT7prestin B residues 638-744; and pGBK-prestin C residues 499-744. All plasmids were confirmed by sequencing analysis. The prestin polypeptides, fused to either the yeast GAL4 DNA-binding or GAL4 transcription activation domain, were introduced sequentially into the S. cerevisiae AH109 yeast cells by the conventional lithium-acetate or commonly known as the LiAc method [110]. The cells were centrifuged at 1,000X rcf, resuspended in $10 \mathrm{mM}$ Tris-EDTA pH 7.0 buffer (see Appendix B), and incubated on $\mathrm{SD}^{-2} /$-Trp-Leu plates at $30^{\circ} \mathrm{C}$ for $48 \mathrm{~h}$, then replicated onto $\mathrm{SD}^{-3} /$-Trp-Leu-His $+15 \mathrm{mM} 3$ AT. 


\section{Chapter 3 Functional Test and Characterization of the Disordered Loop}

\subsection{Residues 571-635 Contribute to Prestin's Voltage Sensor}

NLC represents a property specific to prestin in the membrane [73]. Previous studies have shown that the $\mathrm{C}$-terminus plays a critical role for prestin function. Deletion of the last 32 amino acids of the C-terminus results in loss of non-linear membrane capacitance [69]. To test the role of the C-terminal loop, the wild type prestin (PrestineYFP 1-744) and prestin with residue 571-635 deleted (Prestin-eYFP $\Delta 571-635$ ) were transfected to HEK 293T cells. A four-residue linker (GAAG) replaced the 65 residues corresponding to the loop. Patch clamp experiments using transfected cells provide results comparable to isolated OHCs. Prestin's function is assayed by voltage-clamping a cell and recording the movement of charge as non-linear capacitance $[42,111]$. NLC measurements were made from voltage-clamped HEK 293T cells. The prestin transfected cells had NLC compared to non-transfected HEK 293T. Both Prestin-eYFP 1-744 and Prestin-eYFP $\Delta 571-635$ transfected cells displayed NLC the membrane potential was changed (see Figure 3-1). It can be seen that the sharpness of the NLC curve for the Prestin-eYFP $\triangle 571-635$ broadened in comparison to the Prestin-eYFP 1-744 fusion prestin. The exact values measured for the charge density, voltage at peak capacitance $\left(\mathrm{V}_{\text {peak }}\right)$, and the slope factor ( $\alpha$-value) can be found in Table 3-1. The charge density and $\mathrm{V}_{\text {peak }}$ did not exhibit a significant different between proteins. Charge density equates to the total charge measured $\left(\mathrm{Q}_{\max }\right)$ divided by the linear capacitance $\left(\mathrm{C}_{\text {lin }}\right)$ of the cell. It is a value used to compare prestin activity between different cells. Thus, it gives assurance that the results are a measure of prestin function independent of the cell. The $\alpha$-value of Prestin-eYFP $\Delta 571-635$ had a $10 \mathrm{mV}$ difference $(\mathrm{P}<0.001)$ compared to Prestin-eYFP 1744. The $\alpha$-value is the slope factor, which describes the voltage dependence of the charge transferred. The increased $\alpha$-value for Prestin-eYFP $\Delta 571-635$ indicates that more voltage is required to 'push' the charge movement. The $\alpha$-value for Prestin-eYFP 1-744 was consistent with the wild type prestin in other reports [112].

\subsection{Summary}

The ability to measure NLC in Prestin-eYFP $\Delta 571-635$ indicates that the prestin mutant was correctly targeted to the plasma membrane. This conclusion is based on reports showing that prestin function is innate to the cell's plasma membrane $[113,114]$. Mutations that disrupt prestin's structure also disable the functional ability $[53,69,112]$. The NLC data collected for the loop deleted prestin implies that residues 571-635 may not be necessary for the core structure. This would indicate that the loop splits the folding of the STAS domain into two regions within the sequence. No significant difference in charge density and $\mathrm{V}_{\text {peak }}$ were observed, which indicates that loop deleted prestin underwent conformational changes. The $\alpha$-value for Prestin-eYFP $\Delta 571-635$ increased approximately $10 \mathrm{mV}$ over the $\alpha$-value of wild type. From this data, it can be concluded that the loop has a functional role tied to prestin's voltage sensor. 


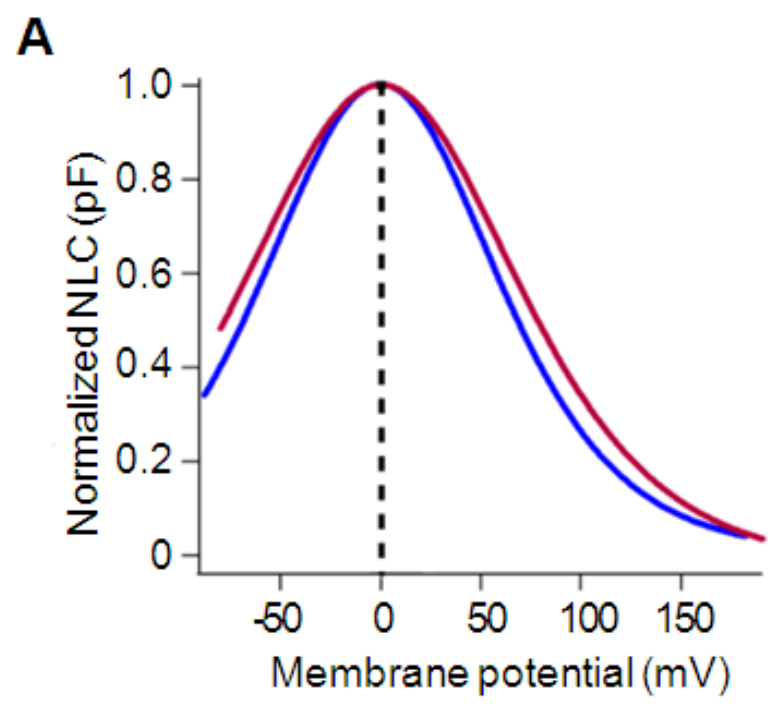

B

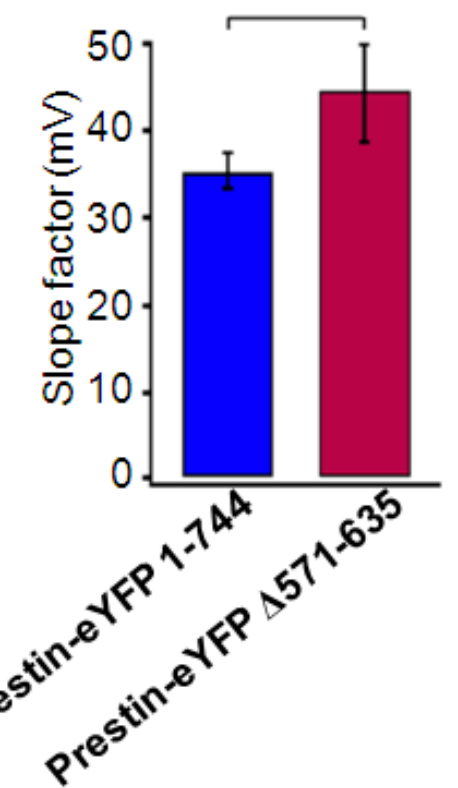

C

Prestin-eYFP 1-744

Prestin-eYFP $\Delta$ 571-744
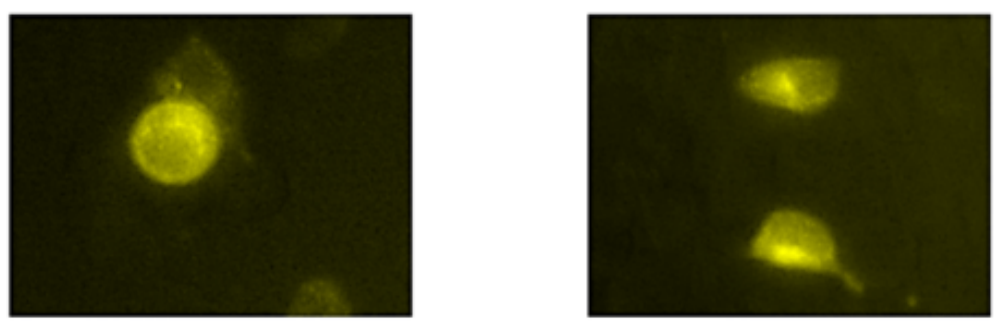

Figure 3-1 Full length prestin $\Delta 571-635$ has an altered voltage response

Deletion $(\Delta)$ of the loop (residues 571-635) from prestin increases the $\alpha$-slope factor in transfected HEK 293T cells. (A) Arrangement of normalized NLC from Prestin-eYFP 1744 and Prestin-eYFP $\Delta 571-635$ together shows that the loop $\Delta$ widens the trace. This outcome reflects a diminished sensitivity to the membrane voltage. The nonlinear capacitance $\mathrm{C}_{(\zeta)}$ data were fitted by a derivative of the two-state Boltzmann function (Eq. 1) that involves linear capacitance $C_{\text {lin }}$, maximum nonlinear charge transfer $Q_{\max }$, the voltage at peak capacitance $V_{1 / 2}$, and slope factor $(\alpha)$ that describes the voltage dependence of the transferred charge from the curve. (B) The loop area in prestin's Cterminus contributes to the voltage sensor function based on a $10 \mathrm{mV}$ increase $(\mathrm{P}<0.001)$ of the $\alpha$-slope factor. There was not a significant difference in charge density and voltage at peak capacitance. (C) Confocal images of Prestin-eYFP 1-744 and Prestin-eYFP $\Delta 571$ 635 indicating placement in the membrane consistent with published reports. 
Table 3-1 NLC data for wild type and $\mathbf{\Delta 5 7 1 - 6 3 5}$ prestin molecules

\begin{tabular}{|c|c|c|c|c|}
\hline Protein & $\begin{array}{l}\text { Slope factor } \\
(\mathrm{mV})\end{array}$ & $\begin{array}{l}V_{1 / 2} \\
(\mathrm{mV})\end{array}$ & $\begin{array}{l}Q_{\max } \\
(f C)\end{array}$ & $\begin{array}{l}\mathbf{Q}_{\max } / \mathbf{C}_{\text {lin }} \\
(\mathbf{f C} / \mathbf{p F})\end{array}$ \\
\hline $\begin{array}{l}\text { Prestin-eYFP } \\
\Delta 571-635\end{array}$ & $\begin{array}{l}44.3 \pm 5.5 \\
\mathrm{P}=4.7 \times 10^{-6}\end{array}$ & $\begin{array}{l}-68.7 \pm 6.4 \\
\mathrm{P}=0.68\end{array}$ & $\begin{array}{l}83.1 \pm 9.7 \\
\mathrm{P}=0.08\end{array}$ & $\begin{array}{l}8.86 \pm 4.32 \\
\mathrm{P}=0.0197\end{array}$ \\
\hline $\begin{array}{l}\text { Prestin-eYFP } \\
1-744\end{array}$ & $35.3 \pm 2.0$ & $-62.6 \pm 10.4$ & $108.2 \pm 15.2$ & $5.23 \pm 2.20$ \\
\hline
\end{tabular}




\section{Chapter 4 Insights to C-terminus Folding and Stas Domains Boundaries}

\subsection{Deletion Mutants Used to Study Folding}

The ability to measure NLC in Prestin-eYFP $\triangle 571-635$ implies that residues 571635 do not fulfill a primarily structural role in the C-terminus or the full-length protein. This would suggest that these residues might exist as a flexible linker between two structured regions. Structure prediction supports this conclusion and also hints that approximately the last 35 residues (710-744) in the sequence do not fold (see Figure 1-7). Additional insight comes from the sequence analysis, which indicates that the loop sequence and residues 710-744 do not constitute part of the STAS domain (see Figure 1-6). Other reports have also suggested that the STAS domain in related SLC26 proteins contains additional sequence $[74,75]$. The domain originates from the sequence flanking these two regions; thus by default, these regions make-up the structure in the C-terminus. Figure 1-5 presents a model of this idea in relation to prestin's transmembrane segments. These two areas of sequence are referred to as STAS A and STAS B. The two regions of predicted disorder essentially section the C-terminus into five different parts relative to STAS A and B. Based on this idea, five different regions or structural motifs (with approximate boundaries) were predicted for the C-terminus: 499-525 (linker sequence from the last transmembrane helix), 526-570 (STAS A), residues 571-635 (loop), residues 636-709 (STAS B), and finally residues 710-744 (disordered C-terminal tail). The layout of the proposed motifs in the C-terminus can be seen in Figure 4-1. To understand the structural organization and folding relative to these five regions in the $\mathrm{C}$ terminus, nine different proteins were made (see Table 4-1). The protein with residues 499-744 constituted the entire cytosolic portion of prestin's C-terminus; thus, it was considered as the wild type or unmutated form. Six of the proteins had sequence deleted from the three areas flanking the STAS A and STAS B (499-525, 571-635, or 710-744). The protein called '526-744 polar' contained the entire loop sequence; however, point mutations were introduced to neutralize an acidic cluster located between 608-613. The polar amino acids, glutamine and asparagine, replaced the native sequence of EEEDDE (glutamic acid and aspartic acid) with QQQNNQ. A seventh protein, 512-712 $\Delta 571-635$, had sequence deleted from all three areas; however, the $\mathrm{N}$-terminal and $\mathrm{C}$-terminal deletion was slightly shorter than the other proteins. The final protein, named STAS B 613-744, had residues 499-612 deleted. Sequence analysis indicates that residues 636-709 pertain to the latter half of the STAS domain after the loop. Thus, this area should form the main structure in this construct. Experiments were designed to study the importance of these five areas to $\mathrm{C}$-terminus structure.

\subsection{Limited Proteolysis Supports the Disorder Predicted for Residues 571-635}

Disordered areas within a protein that lack structure, such as a random coil or loop, often reside on the external surface where they are exposed to the hydrophilic environment [115]. This allows these sites to be accessed for different types of enzymatic modification within the cell. In particular, these regions exhibit susceptibility 


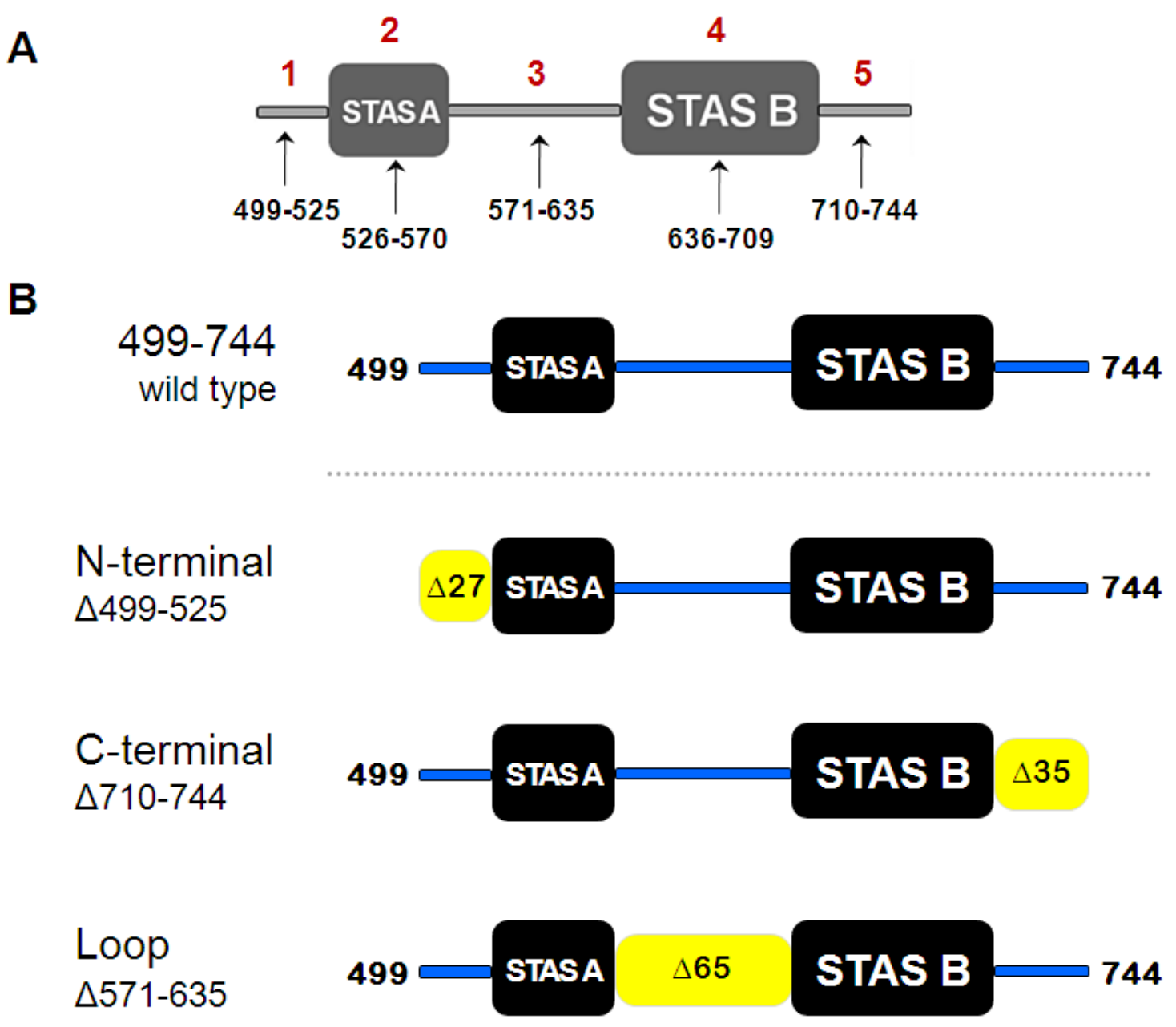

Figure 4-1 Deletion mutants used to isolate the core fold in the C-terminus

(A) Five areas of sequence (red numbers) conceived for studying the structural features of the C-terminus. The sequence boundaries for each area are indicated below (black arrows). (B) Three areas of sequence were deleted (on left) from the C-terminus to investigate the layout of the five proposed structural motifs, relative to STAS A and STAS B. The areas in yellow indicate the location and the number of residues deleted from the C-terminus. These deletions also served to investigate the importance of each motif to the C-terminus structure. 
Table 4-1 Proteins used to study the C-terminus structural properties

\begin{tabular}{ll}
\hline Area deleted & Protein/DNA construct \\
\hline Wild type & $499-744$ \\
$\Delta 499-525$ & $526-744$ polar \\
& $526-744 \Delta 571-635$ \\
$\Delta$ loop & $499-744 \Delta 601-606$ \\
& $499-744 \Delta 601-618$ \\
& $499-744 \Delta 571-635$ \\
$\Delta 710-744$ & $499-709 \Delta 571-635$ \\
$\Delta 499-511+713-744$ & $512-712 \Delta 571-635$ \\
$\Delta 499-612$ & STAS B $613-744$ \\
\hline
\end{tabular}


to protease cleavage [116]. By limiting the enzyme's rate of digestion, it is possible to identify areas in a protein that are disordered or folded based on the resulting peptide fragments that accumulate $[117,118]$. To distinguish between folded and unfolded areas within prestin's C-terminus, limited proteolysis experiments were performed to confirm the disorder predicted for residues 560-636 (see Figure 1-7) and to help identify the boundaries of the STAS A and B subdomains.

The first experiment used a modified form of trypsin to digest the 499-744 wild type protein and a control protein (499-744 $\Delta 601-618)$ that had 18 residues deleted from the predicted loop. The trypsin was designed to cut specifically after lysine or arginine residues. The loop (571-635) has seven lysine and four arginine residues. It was predicted that trypsin would primarily cleave the protein at these residues if they were surfaceexposed and unfolded. To verify that residues 571-635 were not folded, trypsin digested the proteins for 60 minutes. Stable bands formed within two minutes after addition of trypsin. These bands persisted until the experiment was finished at 60 minutes (see Figure 4-2A). Two 10\% Bis-Tris gels are shown for each protein from two separate experiments. The second experiment was performed to monitor progression of the digest at an earlier time point (two minutes). A duplicate of each gel was run at the same time, which was transferred to a PVDF membrane. Four bands were excised from a single lane for both proteins and submitted to direct N-terminal Edman degradation sequencing analysis. Based on the size of the digest fragment and the gel's resolution, each band may have contained more than one digest product. The residues identified were matched with the C-terminus sequence to pinpoint where trypsin cleaved.

The relative position where trypsin cut in the C-terminus can be seen in Figure 4-2B. The N-terminal residues sequenced from the four bands indicated that the digest products likely originated from cleavage that primarily occurred in the disordered loop (see Table 4-2). It appeared that trypsin cleaved at lysine or arginine residues located in the same place for in both proteins. This suggests that both proteins folded similarly and that the loop remained unfolded. It is also worth noting that both proteins displayed a similar banding pattern of digest fragments in the gel. This provides further evidence showing that deletion of 601-618 in the second protein did not affect the structure as these residues reside in the loop. Furthermore, the fact that the digest bands appeared immediately and remained over 60 minutes implies that the peptide fragments correspond to core structure in the $\mathrm{C}$-terminus. Although lacking direct evidence, the data supports the idea of STAS A (526-570) and STAS B (636-709) forming the underlying structure. Collectively, trypsin proteolysis results support the hypothesis that a 60-70 residue loop partitions the STAS into two subdomains. Hereafter, residues 571-635 equate to the primary sequence of the loop.

The results from the trypsin digest provide evidence supporting that the loop is unfolded, while the sequence associated with STAS A and STAS B is structured. However, it is possible that the loop is partially folded with lysine or arginine residues still exposed to trypsin. To further show that the loop is unfolded and it resides between the folded structured of STAS A and B, the C-terminus was digested with the nonspecific protease subtilisin. Subtilisin is a serine endopeptidase which exhibits broad 


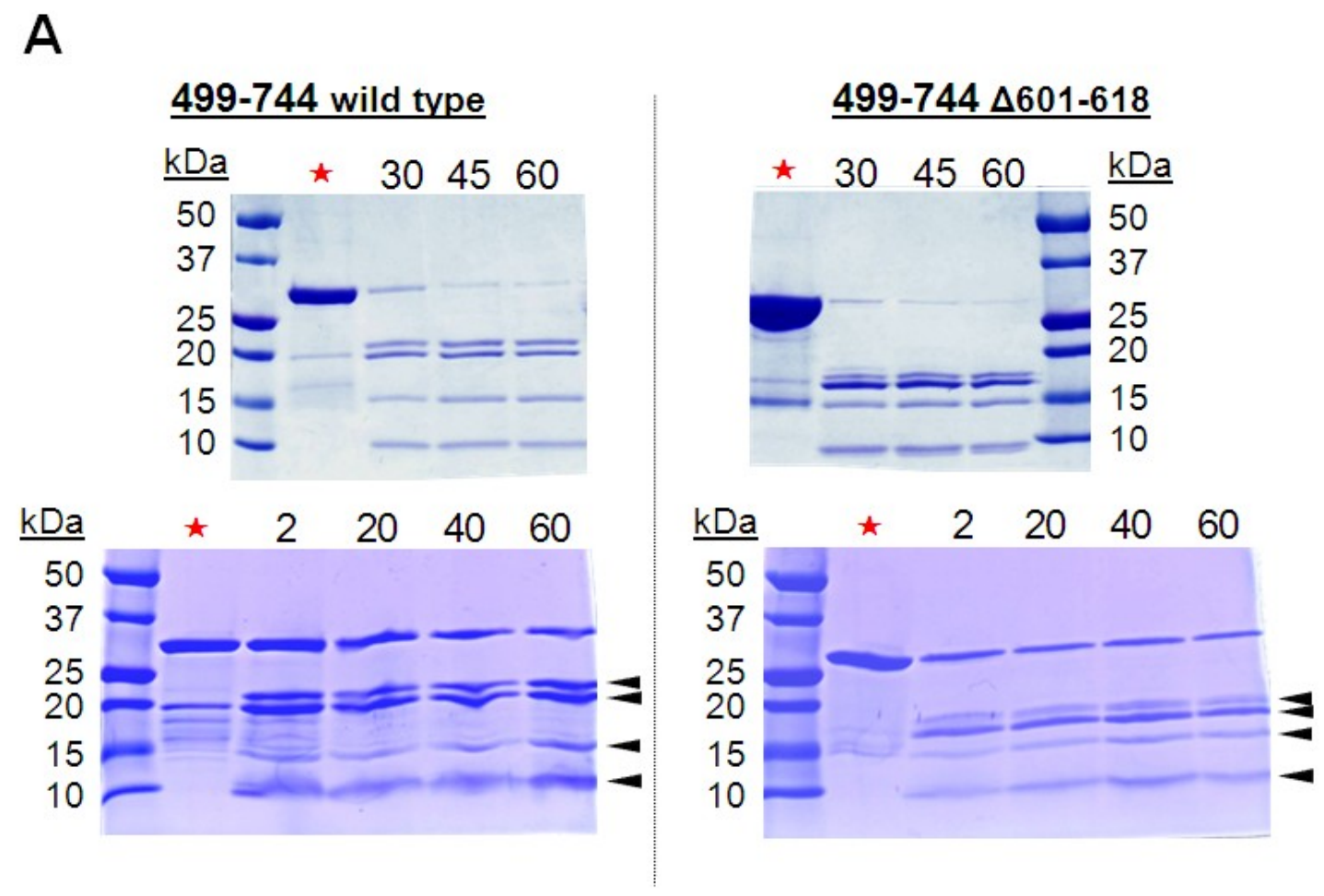

B

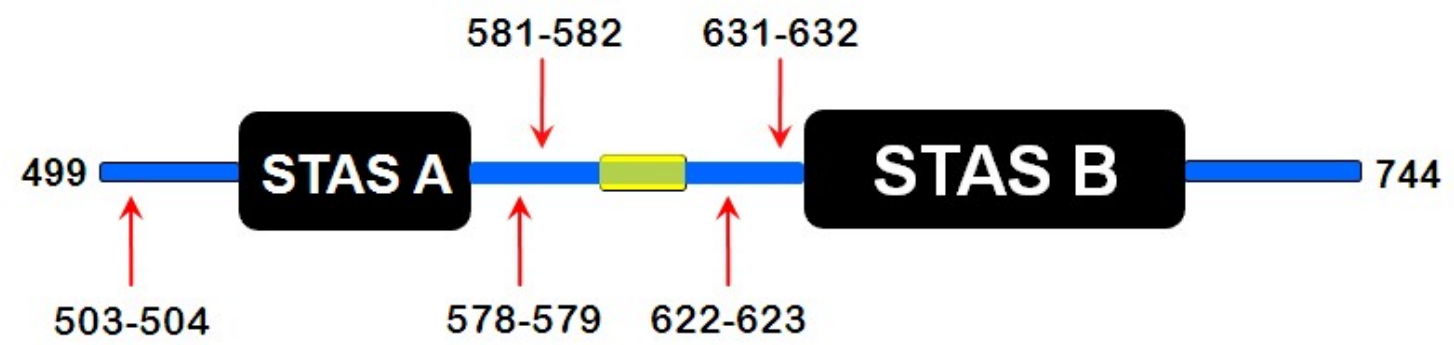

Figure 4-2 Primary 'cuts' from trypsin digestion occurred in the loop region (A) The 499-744 wild type and 499-744 $\Delta 601-618$ proteins were digested with trypsin (1:50 ratio) over one hour. Four stable bands formed within two minutes and persisted over 60 minutes until the experiment's end. The lower gel for each protein corresponds to the second digest attempt where an earlier time point was taken after two minutes. The first lane in each gel, marked with a red star, did not have trypsin added. The black triangles indicate the four gel bands which were sequenced. (B) Direct amino-terminal (Edman) sequencing of the digested products revealed that trypsin cut either near the protein's N-terminus or within the predicted loop. Cuts occurred in approximately the same locations in both proteins. The yellow square indicates the location of the 601-618 deletion relative to where trypsin cut. The results supported the prediction that a 60-70 residue loop divides the structure of the STAS domain in the C-terminus. Red arrows indicate the sites that trypsin cleaved in both proteins. 
Table 4-2 Edman degradation sequence for trypsin cut-sites

\begin{tabular}{lll}
\hline Protein & Residues identified & Sequence location \\
\hline $499-744$ & GSGVIYRTQ & $499-504$ \\
TQSPSYKVL & $503-511$ \\
KYAKEVGNA & $578-585$ \\
LPQGENVH & $632-640$ \\
YAKEVGNA & $579-585$ \\
EVGNANVA & $581-588$ \\
$499-744 \Delta 601-618$ & TTFPEELQ & $623-630$ \\
& GSGVIYRTQ & $499-504$ \\
& TQSPSYKVL & $503-511$ \\
& KYAKEVGNA & $578-585$ \\
& YAKEVGNA & $579-585$ \\
\hline
\end{tabular}


specificity to cleave peptides bonds. In particular, it can hydrolyze peptide bonds at sites with a neutral or acidic amino acid in the P1 position [119]. The presence of glycine in the P1' can also facilitate cleavage [120].

Subtilisin digests were performed on the 499-744 and 499-744 $\Delta 601-606$ proteins. Preparation of these two proteins was identical to the other C-terminus proteins; however, they had six extra residues (GSPEFR) on their N-terminus from cloning (see Table 2-6). Both proteins had three digests set-up with $0.1 \%, 0.5 \%$, or $1 \%$ subtilisin $(\mathrm{w} / \mathrm{w})$ added as a percentage of the total C-terminus protein in the tube $(25 \mu \mathrm{g})$. The reactions were terminated after 60 minutes and analyzed with mass spectroscopy (data not shown). Based on the broad specificity, varying the amount of enzyme provides a way to assess the stability of the resulting peptide fragments. Insufficient enzyme will not adequately digest the sample. An excess of enzyme will produce short, peptide fragments that are not resolvable. This occurred in the digests containing $0.5 \%$ and $1 \%$ subtilisin for both 499 744 and 499-744 $\Delta 601-606$. It was reasonable to assume that most of the structural aspects pertaining to the original protein were lost.

The samples digested with $0.1 \%$ subtilisin produced stable peptides (see Table $4-3$ and Table 4-4). The wild type 499-744 digest had four peptides, which resulted from only two cuts made within the loop between 588-589 and 591-592. Eight peptides resulted from the 499-744 $\Delta 601-606$ digest. Six of the peptides resulted from cuts made to the loop. Two of the fragments resulted from cleavage at two spots (588-589 and 591-592) identical to the cut-sites in the 499-744 protein. Interestingly, a single peptide resulted from two cuts made to sequence associated with the predicted ends of the STAS domain. The loop sequence in this fragment remained intact. Three peptides originated from two cuts made to areas of internal sequence. The first one resulted from cuts between 534-535 and 663-664. Both cleavages occurred just inside the predicted boundaries for the STAS A (526-570). The other two peptides derived from cuts in the middle of the loop (614-615 or 618-619) at their N-terminus and a second cut near the predicted end of the STAS B domain (695-696 or 699-700). Figure 4-3 shows the location of the cleavage site in relation to the predicted STAS A and STAS B.

The data from the subtilisin digest seems to parallel the results from the trypsin digest. Together, both experiments support the hypothesis that the loop region is unfolded. As a result, the loop does not appear to have a discernable role tied to the overall structure. Subtilisin digestion also created produced several internal peptide fragments with boundaries that roughly coincided to the predicted STAS boundaries. Interestingly, the digestion did not produce any peptides from cuts in the middle of STAS A or B. This fact suggests that the actual peptide generated must correspond to the approximate structural boundaries of the STAS domain. Less stringent proteolysis experiments would be required to provide evidence that is more definitive considering that the $0.5 \%$ and $1 \%$ digestions completely fragmented the protein. 
Table 4-3 Stable peptides from subtilisin digest of 499-744

\begin{tabular}{|c|c|c|}
\hline Peptide MW & Residues & Sequence of cleavage fragment \\
\hline 27,871 & control & $\begin{array}{l}\text { gspefrviyrtqspsykvlgqlpdtdvyididayeevkeipgikifqinapiyyansdl } \\
\text { yssalkrktgvnpalimgarrkamrkyakevgnanvanatvvkvdaevdgenatk } \\
\text { peeeddevkfppivikttfpeelqrflpqgenvhtvildftqvnfvdsvgvktlagivk } \\
\text { eygdvgiyvylagcspqvvndltrnnffenpalkellfhsihdavlgsqvreamaeq } \\
\text { eataslpqedmepnatpttpea }\end{array}$ \\
\hline 18,664 & $575-744^{a}$ & $\begin{array}{l}\text { mrkyakevgnanvanatvvkvdaevdgenatkpeeeddevkfppivikttfpeel } \\
\text { qrflpqgenvhtvildftqvnfvdsvgvktlagivkeygdvgiyvylagcspqvvnd } \\
\text { ltrnnffenpalkellfhsihdavlgsqvreamaeqeataslpqedmepnatpttpea }\end{array}$ \\
\hline 10,758 & $499-588$ & $\begin{array}{l}\text { gspefrviyrtqspsykvlgqlpdtdvyididayeevkeipgikifqinapiyyansdl } \\
\text { yssalkrktgvnpalimgarrkamrkyakevgnanva }\end{array}$ \\
\hline 17,131 & $589-744$ & $\begin{array}{l}\text { natvvkvdaevdgenatkpeeeddevkfppivikttfpeelqrflpqgenvhtvildf } \\
\text { tqvnfvdsvgvktlagivkeygdvgiyvylagcspqvvndltrnnffenpalkellfh } \\
\text { sihdavlgsqvreamaeqeataslpqedmepnatpttpea }\end{array}$ \\
\hline 11,045 & $499-591$ & $\begin{array}{l}\text { gspefrviyrtqspsykvlgqlpdtdvyididayeevkeipgikifqinapiyyansdl } \\
\text { yssalkrktgvnpalimgarrkamrkyakevgnanvanat }\end{array}$ \\
\hline 16,845 & $592-744$ & $\begin{array}{l}\text { vvkvdaevdgenatkpeeeddevkfppivikttfpeelqrflpqgenvhtvildftqv } \\
\text { nfvdsvgvktlagivkeygdvgiyvylagcspqvvndltrnnffenpalkellfhsih } \\
\text { davlgsqvreamaeqeataslpqedmepnatpttpea }\end{array}$ \\
\hline
\end{tabular}

${ }^{a}$ Minor degradation product found in the undigested control. 
Table 4-4 Stable peptides from subtilisin digest of 499-744 $\Delta 601-606$

\begin{tabular}{|c|c|c|}
\hline Peptide MW & Residues & Sequence of cleavage fragment \\
\hline 27,273 & control & $\begin{array}{l}\text { gspefrviyrtqspsykvlgqlpdtdvyididayeevkeipgikifqinapiyyansdl } \\
\text { yssalkrktgvnpalimgarrkamrkyakevgnanvanatvvkvdaevdgenatk } \\
\text { peeeddevkfppivikttfpeelqrflpqgenvhtvildftqvnfvdsvgvktlagivk } \\
\text { eygdvgiyvylagcspqvvndltrnnffenpalkellfhsihdavlgsqvreamaeq } \\
\text { eataslpqedmepnatpttpea }\end{array}$ \\
\hline 18,063 & $575-744^{a}$ & $\begin{array}{l}\text { mrkyakevgnanvanatvvkvdaevdpeeeddevkfppivikttfpeelqrflpq } \\
\text { genvhtvildftqvnfvdsvgvktlagivkeygdvgiyvylagcspqvvndltrnnf } \\
\text { fenpalkellfhsihdavlgsqvreamaeqeataslpqedmepnatpttpea }\end{array}$ \\
\hline 21,254 & $513-708$ & $\begin{array}{l}\text { qlpdtdvyididayeevkeipgikifqinapiyyansdlyssalkrktgvnpalimga } \\
\text { rrkamrkyakevgnanvanatvvkvdaevdpeeeddevkfppivikttfpeelqrf } \\
\text { lpqgenvhtvildftqvnfvdsvgvktlagivkeygdvgiyvylagcspqvvndltr } \\
\text { nnffenpalkellfhsihd }\end{array}$ \\
\hline 13,638 & $535-663$ & $\begin{array}{l}\text { ikifqinapiyyansdlyssalkrktgvnpalimgarrkamrkyakevgnanvanat } \\
\text { vvkvdaevdpeeeddevkfppivikttfpeelqrflpqgenvhtvildftqvnfvds } \\
\text { vgvktlagi }\end{array}$ \\
\hline 16,244 & $562-714$ & $\begin{array}{l}\text { vnpalimgarrkamrkyakevgnanvanatvvkvdaevdpeeeddevkfppivi } \\
\text { kttfpeelqrflpqgenvhtvildftqvnfvdsvgvktlagivkeygdvgiyvylagc } \\
\text { spqvvndltrnnffenpalkellfhsihdavlgsq }\end{array}$ \\
\hline 16,531 & $589-744$ & $\begin{array}{l}\text { natvvkvdaevdpeeeddevkfppivikttfpeelqrflpqgenvhtvildftqvnfv } \\
\text { dsvgvktlagivkeygdvgiyvylagcspqvvndltrnnffenpalkellfhsihdav } \\
\text { lgsqvreamaeqeataslpqedmepnatpttpea }\end{array}$ \\
\hline 16,244 & $592-744$ & $\begin{array}{l}\text { vvkvdaevdpeeeddevkfppivikttfpeelqrflpqgenvhtvildftqvnfvds } \\
\text { vgvktlagivkeygdvgiyvylagcspqvvndltrnnffenpalkellfhsihdavlg } \\
\text { sqvreamaeqeataslpqedmepnatpttpea }\end{array}$ \\
\hline 9,091 & 615-695 & $\begin{array}{l}\text { kfppivikttfpeelqrflpqgenvhtvildftqvnfvdsvgvktlagivkeygdvgIy } \\
\text { vylagcspqvvndltrnnffen }\end{array}$ \\
\hline 9,031 & $619-699$ & $\begin{array}{l}\text { ivikttfpeelqrflpqgenvhtvildftqvnfvdsvgvktlagivkeygdvgIyvyla } \\
\text { gcspqvvndltrnnffenpalk }\end{array}$ \\
\hline 13,423 & $623-744$ & $\begin{array}{l}\text { ttfpeelqrflpqgenvhtvildftqvnfvdsvgvktlagivkeygdvgiyvylagcs } \\
\text { pqvvndltrnnffenpalkellfhsihdavlgsqvreamaeqeataslpqedmepna } \\
\text { tpttpea }\end{array}$ \\
\hline
\end{tabular}

${ }^{a}$ Minor degradation product found in the undigested control. 


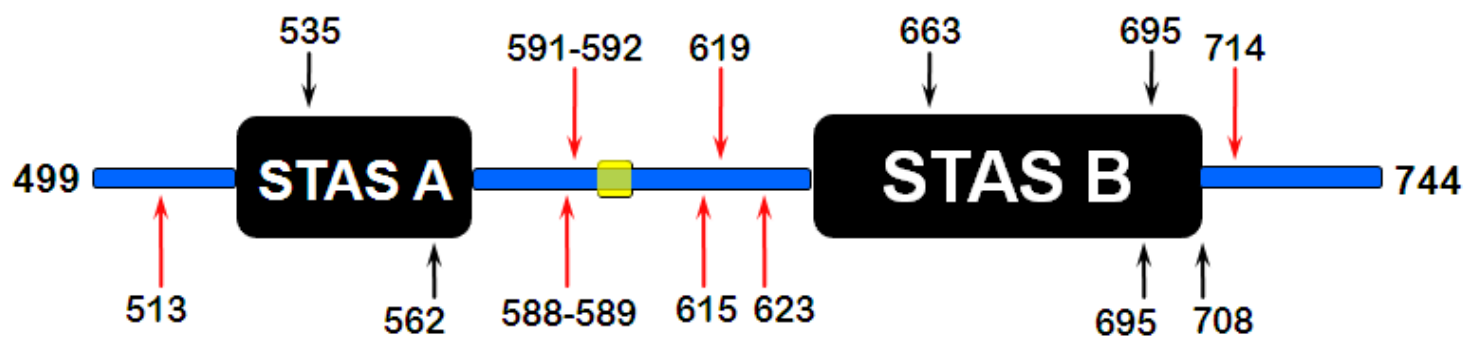

Figure 4-3 Overview of subtilisin cleavage in the C-terminus

Black arrows indicate that cleavage occurred within the predicted STAS sequence. Red arrows highlight the cuts made to the sequence flanking the STAS. These regions are not predicted to fold well. The yellow square indicates the location of the 601-606 deletion. 


\subsection{C-terminus Is Monomeric and Adopts Multiple Conformations}

The proteolysis results provide evidence indicating that the loop is disordered based on its susceptibility to cleavage. Moreover, subtilisin cleavage yielded peptide fragments that approximated the boundaries proposed for the STAS A (526-570) and STAS B (636-709) subdomains. Based on this data, the oligomeric state and structural properties of the C-terminus deletion proteins, described in Figure 4-1, were characterized in solution. Regarding the $\mathrm{C}$-terminus structure, the following specific aims were investigated: to determine whether STAS A and B formed the core structure, provide data to pinpoint the boundaries, and assess the importance of each of the five areas to the overall structure. Two different AUC experiments (SE and SV) were performed. SE, along with size exclusion chromatography-multi-angle light scattering (SEC-MALS), ascertained whether the proteins formed oligomers. SV evaluated the shape (folding conformation), molar mass, and size distribution.

Analytical ultracentrifugation uses centrifugal force to characterize the sedimentation behavior of a protein in solution. The mass, size, and shape of the protein will directly affect the sedimentation rate. Initially, all molecules in the solution are uniformly dispersed. High angular velocity causes proteins and other solutes to migrate towards the bottom of the cell which contains the protein-buffer mixture within the rotor. As the spin progresses, the protein located at the solution's meniscus, near the top of the cell, becomes depleted. A boundary forms that marks the area of depleted of protein (top of the cell) with the area where the protein has concentrated and continues to sediment towards the cell bottom. The speed at which the boundary moves is characterized by its sedimentation coefficient. The sedimentation coefficient is the rate of sedimentation per unit centrifugal field for a particle (protein) [121]. Effectively, the protein concentration increases the greater the distance from the meniscus; however, diffusion also occurs which opposes the sedimentation. This causes the boundary layer to spread out at a rate based on the protein or particle size. The rate at which the boundary spreads is characterized by the diffusional coefficient. In SV experiments, a protein's MW can be calculated from the ratio of the sedimentation to the diffusion coefficient. The overall size and shape of a protein in solution derives from its folding. A protein with a more globular or spherical shape will experience less drag during the sedimentation compared to a protein with an elongated fold and the same MW. The frictional coefficient describes the drag force experienced by a protein dependent upon its size and shape in a fluid (buffer) with a particular viscosity. In these studies, the frictional ratio $\left(f / f_{0}\right)$ is used. The frictional ratio is a hydrodynamic value describing the ratio of the translational frictional coefficient of the particle to that of a spherical particle of the same mass and volume. Typically a hydrated frictional ratio ranges from 1.2 for nearly globular hydrated proteins to 2.0 for very elongated or glycosylated proteins [122]. In the event that a protein has more than one conformation, each population should have the same MW. However, they will each have a different sedimentation coefficient dependent upon the size and shape variation. Over time, heterogeneity in the diffusional rates between s-value can make it difficult to accurately determine the diffusion coefficient and mass. Sedimentation equilibrium experiments provide a means to accurately determine the mass in this circumstance. During sedimentation, the protein's concentration distribution increases as 
the radial distance increase. Diffusion, at each point of the concentration gradient, balances the sedimentation as a function of the MW. A logarithmic plot of the protein's concentration over the radial distance $\left(r^{2}\right)$ reveals the MW, which is the slope.

To characterize the hydrodynamic properties of the C-terminus, SV and SE experiments were performed for 499-744, 526-744, 499-744 $\Delta 601-618,499-744 \Delta 571$ $635,526-744 \Delta 571-635,499-709 \Delta 571-635,512-712 \Delta 571-635$, and STAS B 613-744 proteins. Data for the sedimentation coefficients, frictional ratios, molecular masses, ellipsoid shape and Stokes radius is summarized in Table 4-5. The SV data was analyzed with the $\mathrm{c}(\mathrm{s})$ distribution model, which assumes a single best-fit weight-average frictional ratio $\left(f / f_{0, w}\right)$ value for all species in solution. The $\mathrm{c}(\mathrm{s})$ analysis can provide the range of sedimentation coefficients that describe a mixture of sedimenting macromolecular species with different sizes. The concentration at each s-value, $c(s)$, is obtained from the analysis [105].

SV experiments on all the C-terminus proteins were performed. In all cases, the mutants exist as monomers in solution at the experimental conditions employed. The sedimentation profile for the 499-744 $\Delta 571-635$ protein consistently showed a single major peak as did the profile of the 499-744 protein (see Figure 4-4A and B). Even at high concentration ( $270 \mu \mathrm{M}$ and $430 \mu \mathrm{M}$, data not shown) the 499-744 $\Delta 571-635$ mutant sedimented in a single major peak with a sedimentation coefficient value at $20^{\circ} \mathrm{C}\left(\mathrm{s}_{20}\right.$ value) of $1.73 \mathrm{~S}, \mathrm{f} / \mathrm{f}_{0, w}$ value of 1.43 ; and a $\mathrm{MW}$ of $20.3 \mathrm{kDa}$. The elution profile for 499$744 \Delta 571-635$ from SEC-MALS analysis also showed a single peak which corresponded to a protein of $20.0 \mathrm{kDa}$ (see Figure 4-4C). The single major peak in the sedimentation profile for 499-744 had an $\mathrm{s}_{20}$ value of $2.12 \mathrm{~S}, \mathrm{f} / \mathrm{f}_{0, w}$ value of 1.50 ; and a MW of $27.5 \mathrm{kDa}$. The predicted molar mass for monomeric 499-744 C-term is 27,398 Da and 20, 3 (see Table 2-6). Additionally, data from SE experiments on the 499-744 $\Delta 571-635$ and the 499-744 proteins provides further evidence that the C-terminus does not self-interact. There were no oligomeric species of note observed for either protein (see Figure 4-5A and B). The equilibrium data for 499-744 $\Delta 571-635$ (loading concentration $64 \mu \mathrm{M}$ ) fitted best to a single monomeric species model with a molar mass of 20,314.8 Da. This shows that 499-744 $\Delta 571-635$ does not self-associate or oligomerize under the experimental conditions used. The SE result for 499-744 (loading concentration $30 \mu \mathrm{M}$ ) was more ambiguous. The data fitted well to a two species model with one species fixed at the monomeric molar mass of 27,398.3 Da. The molar mass of the other species was floated and fitted to a molar mass of approximately $60 \mathrm{kDa}$ (around $30 \%$ of total protein). It was concluded that it represented aggregated or contaminating material, which was also observed in the velocity experiments. The equilibrium data do not fit a monomer-dimer self-association model.

The results of the $\mathrm{c}(\mathrm{s})$ analysis, where a single best-fit weight-average frictional ratio $\left(f / f_{0, w}\right)$ is obtained, shows that the 499-744 $\Delta 571-635$ mutant has a frictional ratio of 1.43 in buffer containing salt. The same experiment without salt yielded two populations with the frictional ratios 1.51 and 1.42. A frictional ratio of 1.2 signifies a compact globular protein. In terms of gross hydrodynamic shape, these frictional ratio values were equivalent to a prolate ellipsoid shape with major and minor axes of $11.9 \times 2.5 \mathrm{~nm}$ in salt 
Table 4-5 Summary of SV data for C-terminus proteins

\begin{tabular}{|c|c|c|c|c|c|c|c|}
\hline Protein & $\mathbf{m g} / \mathbf{m l}^{a}$ & $s_{20}^{b}$ & $s_{20, w}{ }^{c}$ & $f / f_{0}{ }^{d}$ & Molar mass $^{e}$ & ${ }^{e}$ Prolate size ${ }^{f}$ & $\begin{array}{l}\text { Stokes } \\
\text { radius } \\
\end{array}$ \\
\hline $499-744$ & 1.27 & $1.95-1.97$ & 2.08 & $1.45(1.53)$ & $\begin{array}{l}25.0-27.0 \\
(27.4)\end{array}$ & $15.5 \times 2.6$ & 3.1 \\
\hline $\begin{array}{l}526-744 \\
\text { polar }\end{array}$ & 0.73 & 1.92 & 2.03 & $1.45(1.45)$ & $24.5(24.3)$ & $13.1 \times 2.6$ & 2.8 \\
\hline $\begin{array}{l}499-744 \\
\Delta 601-618\end{array}$ & 0.75 & 1.95 & 2.06 & $1.45(1.45)$ & $25.0(25.4)$ & $13.5 \times 2.6$ & 2.8 \\
\hline $\begin{array}{l}499-744 \\
\Delta 571-635\end{array}$ & 0.90 & 1.73 & 1.83 & $1.44(1.43)$ & $20.3(20.3)$ & $11.9 \times 2.5$ & 2.6 \\
\hline $\begin{array}{l}499-744 \\
\Delta 571-635^{h}\end{array}$ & $\begin{array}{l}0.43 \\
0.28\end{array}$ & $\begin{array}{l}1.71 \\
1.82\end{array}$ & $\begin{array}{l}1.73 \\
1.84\end{array}$ & $\begin{array}{l}1.55(1.51) \\
1.45(1.42)\end{array}$ & $\begin{array}{l}21.0(20.3) \\
21.0\end{array}$ & $\begin{array}{l}13.7 \times 2.3 \\
11.7 \times 2.5\end{array}$ & $\begin{array}{l}2.7 \\
2.6\end{array}$ \\
\hline $\begin{array}{l}526-744 \\
\Delta 571-635\end{array}$ & $\begin{array}{l}0.22 \\
0.40 \\
0.20\end{array}$ & $\begin{array}{l}1.53 \\
1.57 \\
1.65\end{array}$ & $\begin{array}{l}1.60 \\
1.65 \\
1.73\end{array}$ & $\begin{array}{l}1.55(1.47) \\
1.45(1.43) \\
1.33(1.36)\end{array}$ & $\begin{array}{l}18.6(17.3) \\
17.5 \\
17.0\end{array}$ & $\begin{array}{l}12.0 \times 2.3 \\
11.2 \times 2.4 \\
9.9 \times 2.5\end{array}$ & $\begin{array}{l}2.5 \\
2.4 \\
2.3\end{array}$ \\
\hline $\begin{array}{l}499-709 \\
\Delta 571-635\end{array}$ & 0.16 & 1.59 & 1.67 & $1.28(1.33)$ & $16.0(16.6)$ & $9.4 \times 2.5$ & 2.3 \\
\hline $\begin{array}{l}512-712 \\
\Delta 571-635\end{array}$ & 0.58 & 1.63 & 1.72 & $1.15(1.23)$ & $14.0(15.4)$ & $6.9 \times 2.8$ & 2.0 \\
\hline $\begin{array}{l}\text { STAS B } \\
613-744\end{array}$ & 0.42 & 1.43 & 1.50 & $1.35(1.40)$ & $14.0(14.8)$ & $10.2 \times 2.3$ & 2.3 \\
\hline
\end{tabular}

${ }^{a}$ Protein concentration $(\mathrm{mg} / \mathrm{ml})$ is in parenthesis.

${ }^{b}$ Sedimentation coefficient taken from the ordinate maximum peak of contour plots; $\mathrm{s}$ vs. $f / f_{0}$ distribution $\left(c\left(s, f / f_{0}\right)\right)$ analysis [106].

${ }^{c}$ Converted $s$ values to $s_{20, w}^{0}$ to standard conditions in Svedberg $\left(20^{\circ} \mathrm{C}\right.$, water solvent, zero protein concentration).

${ }^{d}$ Experimental $f / f_{0}$ is from the ordinate maximum peak of contour plots, $s$ vs. $f / f_{0}$ distribution ( $c\left(s, f / f_{0}\right)$ analysis). The $f / f_{0, w}$ calculated with SEDNTERP and standard $s^{0}{ }_{20, w}$ values in parenthesis.

${ }^{e}$ Molar mass values $(\mathrm{kDa})$ taken from the ordinate maximum peak of the contour plot, $\mathrm{s} v s . \mathrm{M}$ distribution $\left(c\left(s, f / f_{0}\right)\right.$ analysis) [106]. The theoretical MW is in parenthesis.

${ }^{f}$ Derived from molar mass and standard $s_{20, w}{ }_{20}$. Two-fold major and minor axis $(\mathrm{nm} \times \mathrm{nm})$ calculated assuming a hydration in $\mathrm{g} / \mathrm{g}$ as predicted from SEDNTERP based on residues.

${ }^{g}$ Stokes radius (nm) calculated from standard $s_{20, w}^{0}$ using SEDNTERP.

${ }^{\boldsymbol{h}}$ Buffer did not contain $\mathrm{NaCl}$. 

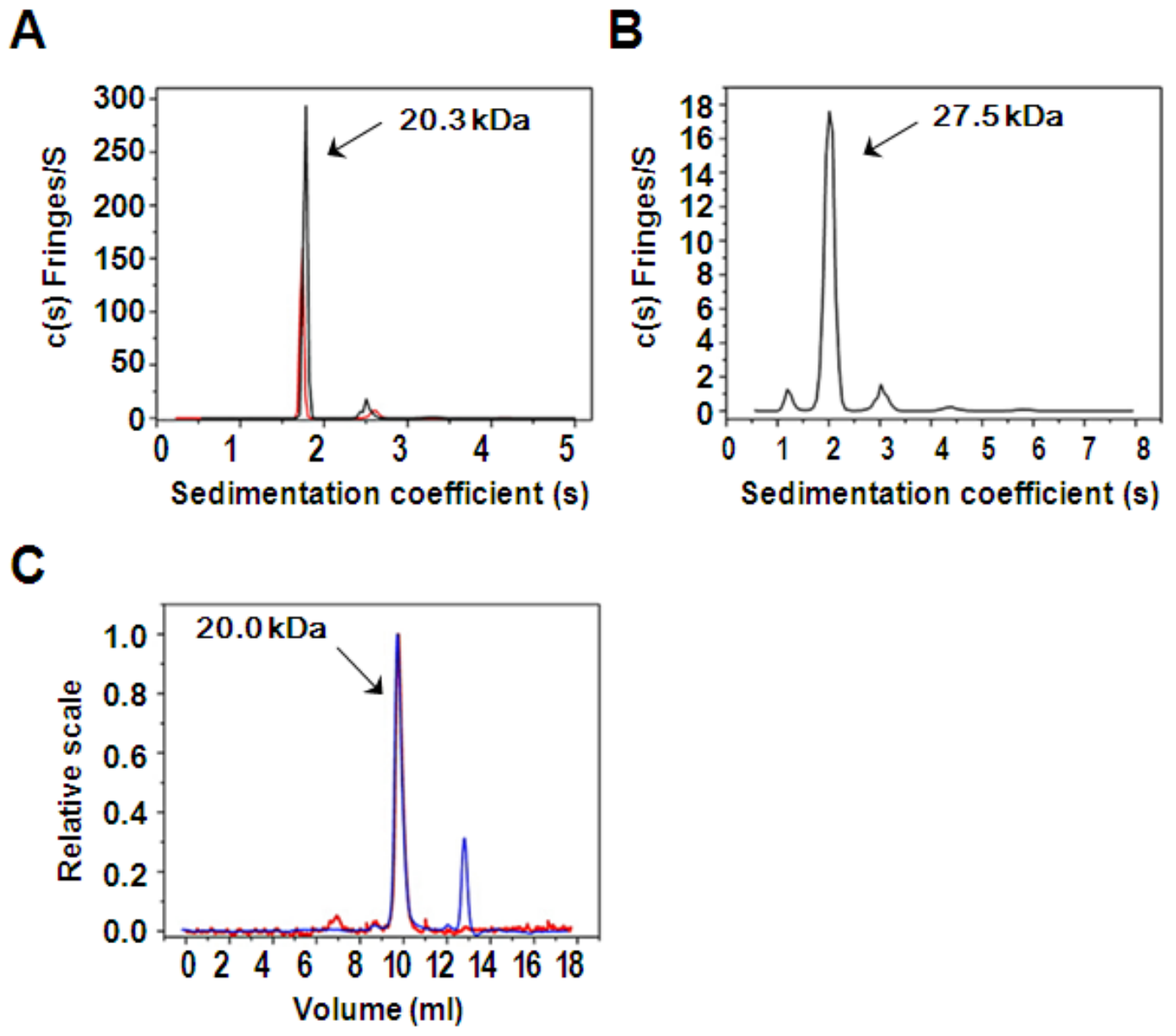

Figure 4-4 SV and SEC-MALS show that the C-terminus does not oligomerize SV profiles (fringe displacement) for 499-744 $\Delta 571-635$ and 499-744, which were fit to a continuous sedimentation coefficient distribution model $\mathrm{c}(\mathrm{s})$, reveal monomeric forms in solution. (A) SV profile of the 499-744 $\Delta 571-635$ protein. Protein concentrations of 150 and $270 \mu \mathrm{M}$ were assessed. The $S_{20, w}^{0}$ value for $499-744 \Delta 571-635$ was $1.86 \mathrm{~S}$, the $f / f_{0, w}$ value was 1.40; and the MW calculated was 20,300 Da. The theoretical MW is 20,314 Da. (B) SV profile of the 499-744 protein. The $S^{0}{ }_{20, w}$ value for 499-744 was $2.12 \mathrm{~S}$, the $f / f_{0, w}$ value was 1.50 , and the MW calculated was 27,500 Da. The theoretical $\mathrm{Mw}$ is 27,399 Da. (C) SEC-MALS data for 499-744 $\Delta 571-635$ protein. Static laser light scattering (red line) and differential refractive index (blue line) data were recorded for the protein in the eluant of a size-exclusion column (exclusion limit 60,000 Da). The flow rate was $0.3 \mathrm{ml} / \mathrm{min}$, loading protein concentration $0.15 \mathrm{mg} / \mathrm{ml}$, and the application volume $50 \mu \mathrm{l}$. The experiment occurred at $25^{\circ} \mathrm{C}$. The peak protein corresponds to the theoretical MW of $20 \mathrm{kDa}$, close to the theoretical MW of 499-744 $\Delta 571-635$. 


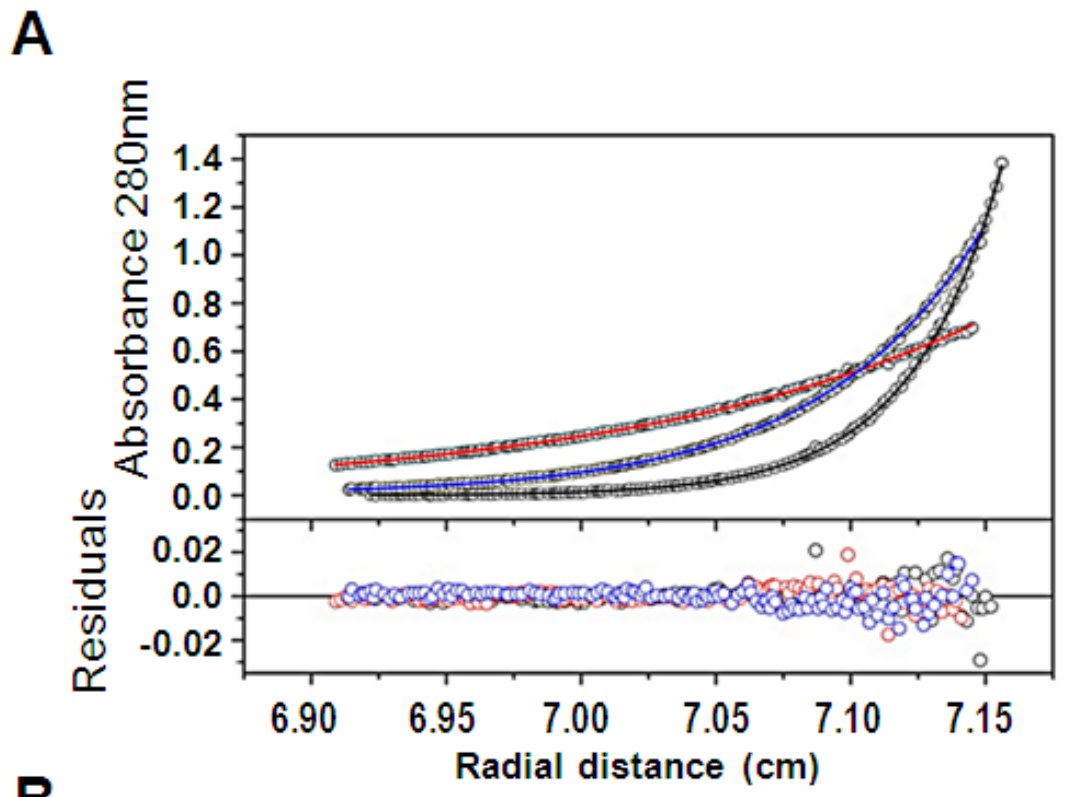

B

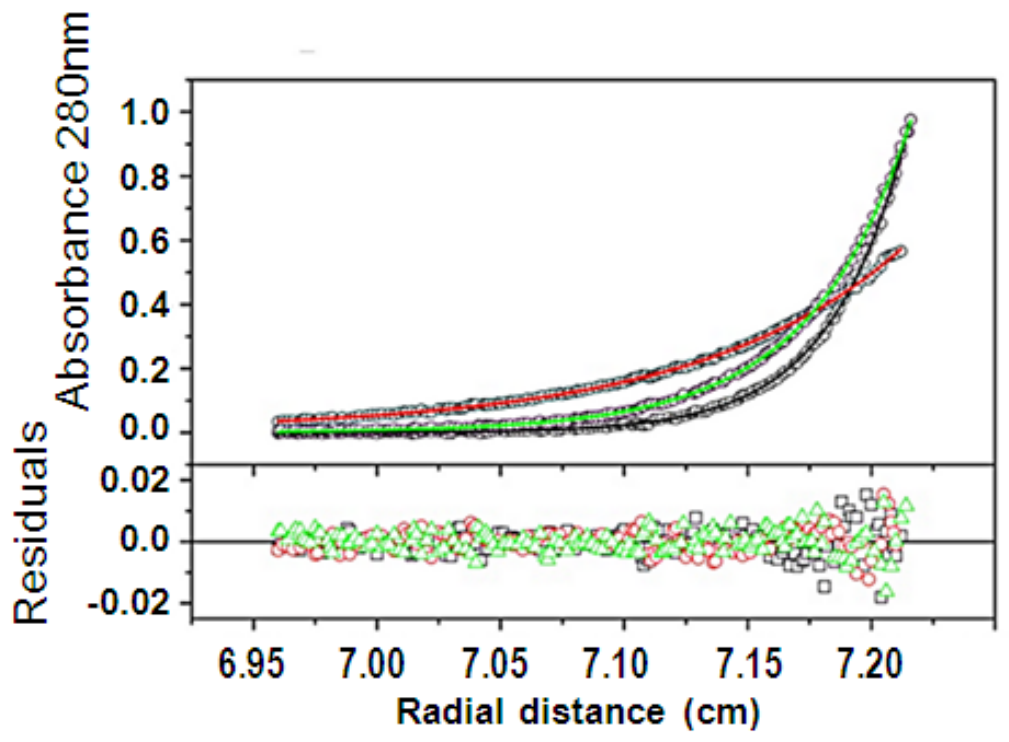

Figure 4-5 SE shows that 499-744 $\Delta 571-635$ and 499-744 are monomers

Absorbance scans at $280 \mathrm{~nm}$ at equilibrium are plotted versus the distance from the axis of rotation. The solid lines represent the global nonlinear least squares best-fit of all the data sets to a single species (499-744 $\Delta 571-635)$ (MW 20,300 Da) and a two discrete species model (499-744 wild type) one 27,399 Da and the other 60,000 Da (30\% of total protein). Residuals of the fit are also shown and the RMSD is between 0.0030-0.0045 absorbance units. The polydispersity factor (1.027) was close to 1.000 , signifying significant monodispersity (i.e. homogeneity) of the sample. (A) SE data for 499-744 $\Delta 571-635$. Red equates to 20,000 , blue to 30,000 , and black to $40,000 \mathrm{rpm}$. The $499-744$ $\triangle 571-635$ protein does not self-associate under the conditions used. (B) SE data for 499744 . Red equates to 20,000 , green to 30,000 , and black to $37,000 \mathrm{rpm}$. 
buffer. For the no salt buffer, the dimensions for the two different populations were $13.7 \times 2.3 \mathrm{~nm}$ and $11.7 \times 2.5 \mathrm{~nm}$, respectively. The hydration ratio $(0.405 \mathrm{~g} / \mathrm{g})$ used to calculate the major and minor axis was estimated from the amino acid composition The dimensions indicate that the protein has a relatively elongated molecular shape. In low salt, the protein appeared to assume en even more extended shape; however, the no-salt condition was not tested for the other proteins. The extended conformation seen for the 499-744 $\Delta 571-635$ protein was also a consistent feature for the other proteins (see Table 4-5)

The c(s) analyses for 499-744, 526-744 polar, and 526-744 $\Delta 571-635$ also displayed some asymmetry in their sedimentation peaks. It is possible this could result from micro-heterogeneity in the protein's mass or from differences in shape (conformation). To investigate whether differences existed with regard to the hydrodynamic friction, a two-dimensional size-and-shape distribution $c\left(s, f / f_{0}\right)$ was calculated with the same velocity data to generate contour plots. The s-value distribution was plotted in one dimension and $f / f_{0}$ distribution in the other. Contour plots of the molar mass (M) vs. the s-value were also created (see Figure 4-6, Figure 4-7, and Figure 4-8). The best-fit values and estimates of the $c\left(s, f / f_{0}\right)$ analyses were determined from the ordinate maximum peaks from the contour plots of $f / f_{0} v s$. s-value and $\mathrm{M} v s$. s-value. This data is summarized in Table 4-5. The M vs. s-value contour plots of the 499-744 (see Figure 4-6A) and the 526-744 polar (see Figure 4-6B) mutant shows that the proteins resolve into single peaks respectively. However, the $f / f_{0} v s$. s-value contour plots reveal that the velocity data fitted best to a wide distribution of $f / f_{0}$ as well as sedimentation coefficient value. This suggests that the molecule exhibits flexibility in solution.

For mutants 499-744 $\Delta 571-635$ and 526-744 $\Delta 571-635$ the molar mass closely agrees with the predicted mass (see Table 2-6), but the shape plots $\left(f / f_{0}\right.$ vs. s-value) show species with dissimilar $f / f_{0}$ values. The 526-744 $\Delta 571-635$ protein is the strongest example. The different frictional ratios suggest that the same protein has different shapes. The 499-744 $\Delta 571-635$ protein appears to have two relatively distinct molecular shapes in the no salt solution, with frictional ratios of 1.42 and approximately 1.51 (see Figure 4-7B). In the salt buffer solution, the $f / f_{0}$ vs. s-value contour peak covers a distribution of frictional ratio values with the peak apex at 1.43 (see Figure 4-7A). Notably, the 526-744 $\triangle 571-635$ also shows value for the frictional ratio distributed from 1.33-1.55 (see Figure 4-7C). This protein only lacks residues $499-525$ in comparison to $499-744 \Delta 571-635$.

Mutants 499-709 $\Delta 571-635,512-712 \Delta 571-635$, and STAS B 613-744 also appear to resolve into single major peaks (see Figure 4-8). The $s_{20}$ values are listed in Table 4-5. Their frictional ratio values vary from 1.15 to 1.35 , which signifies relatively globular shapes. The molecular mass for 512-712 $\Delta 571-635$ (Figure 4-8B) was slightly below the predicted value (Table 2-6). This could result if some of the protein, unaccounted-for in the sample, belongs to a rapidly interconverting system [122]. Such behavior can often be attributed to disorder or flexible sequence within a protein [123, 124]. The GAAG linker, which replaced the loop, was used based on the flexibility conferred by the glycines to the carbon backbone. Overall, the mass values for the other 

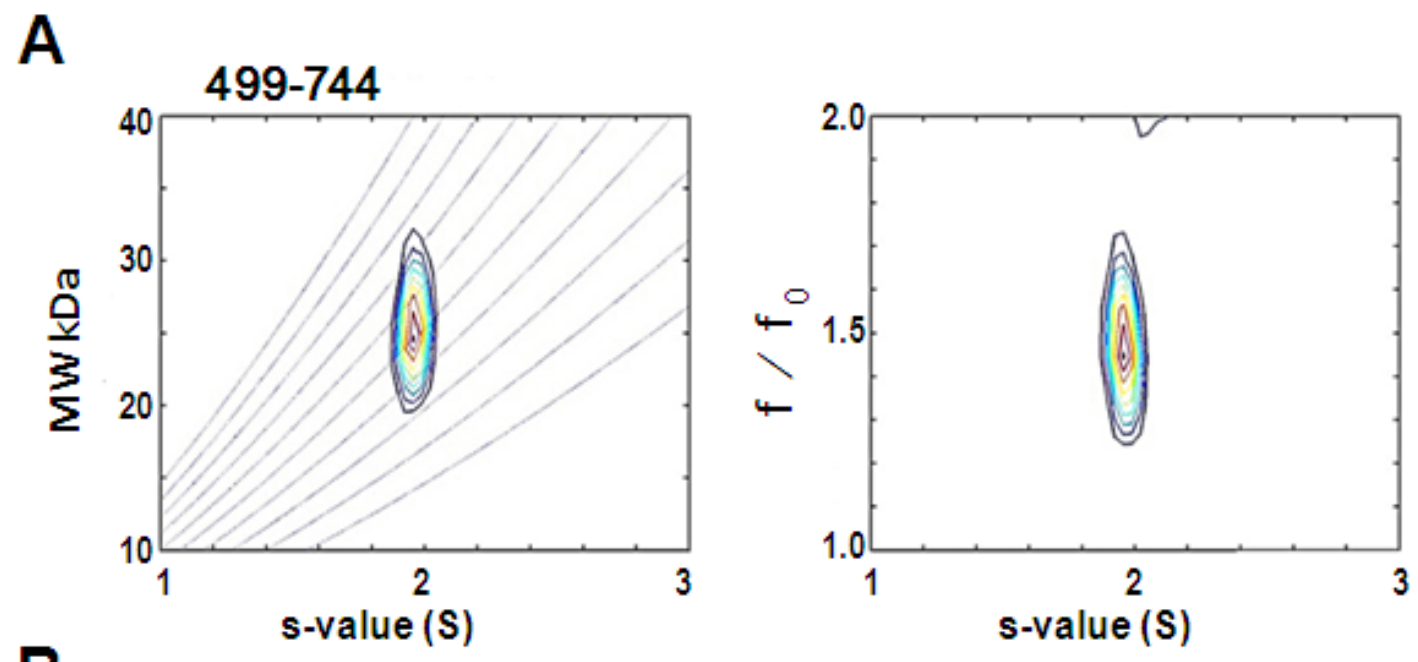

B
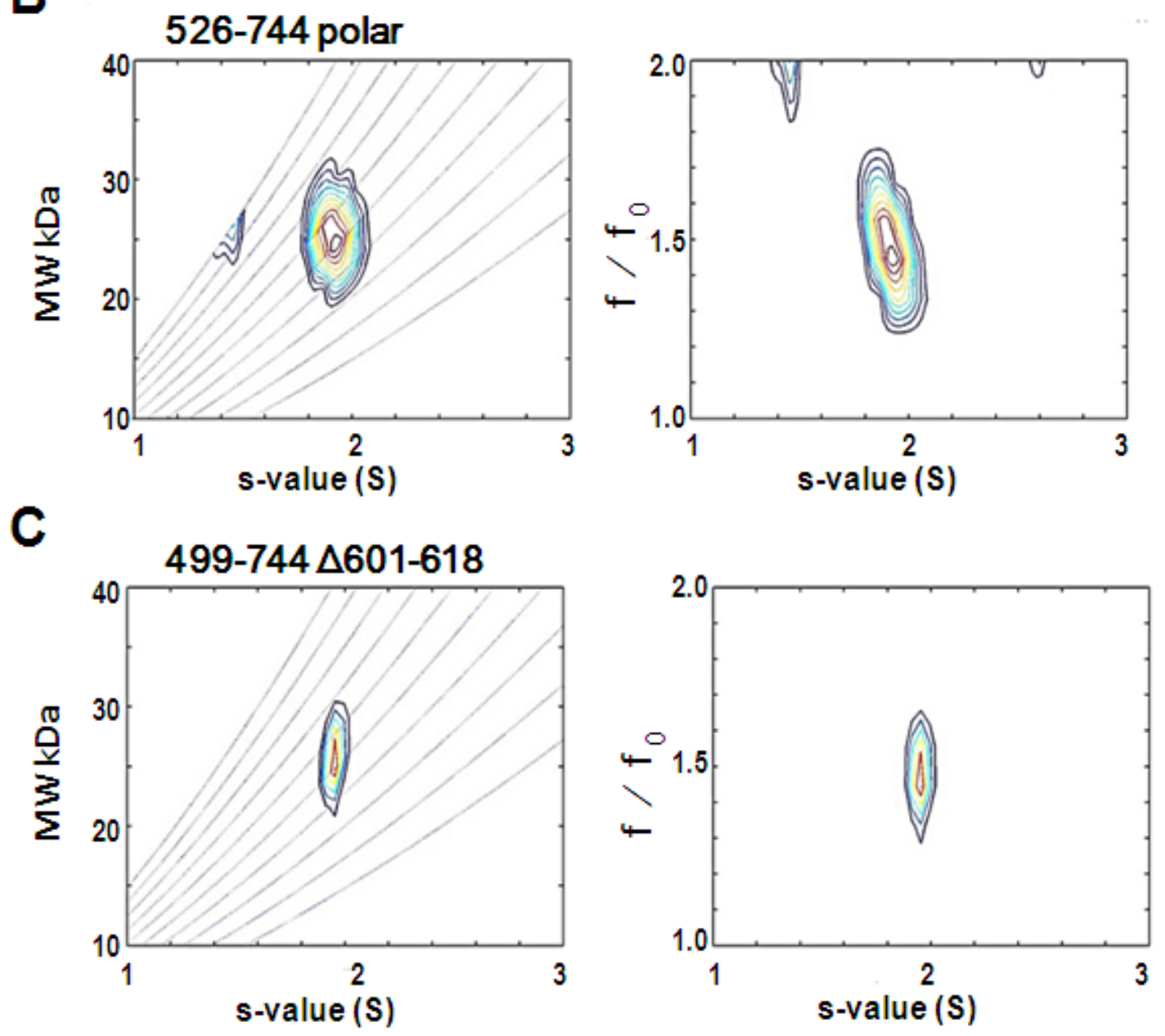

Figure 4-6 2D contour plots: 499-744, 526-744 polar, and 499-744 $\Delta 601-618$

(A) SV results for 499-744. (B) SV results for 526-744 polar. (C) SV results for 499-744 $\Delta 601-618$. All proteins exhibited a molecular mass consistent with a monomer. 

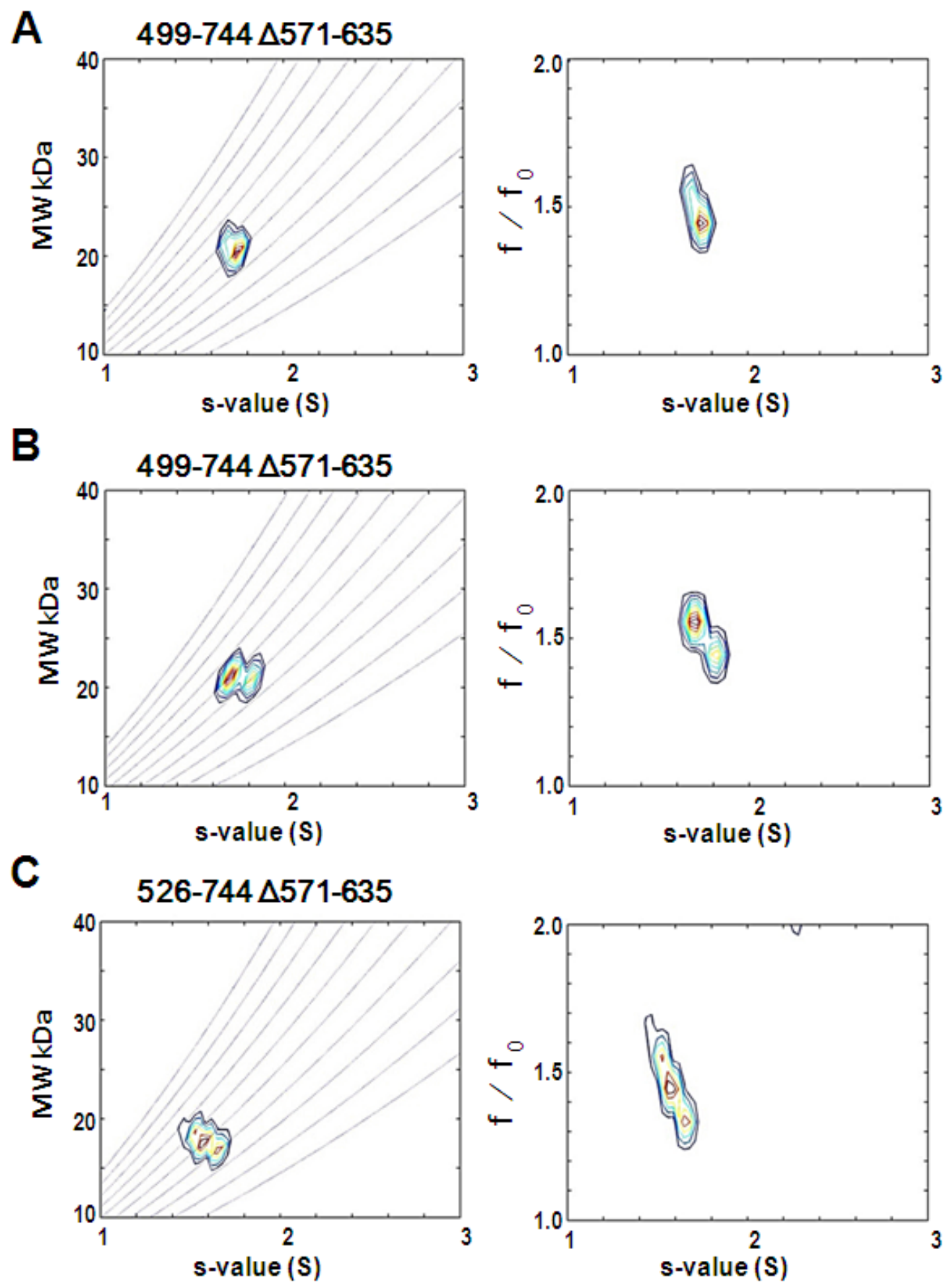

Figure 4-7 2D contour plots: $499-744 \Delta 571-635$ and 526-744 $\Delta 571-635$

(A) SV results for 499-744 $\Delta 571-635$ in $150 \mathrm{mM} \mathrm{NaCl}$ buffer solution. (B) 499-744 $\Delta 571-635 \mathrm{SV}$ results in buffer lacking salt. (C) SV results for 526-744 $\Delta 571-635$. All proteins exhibited a molecular mass consistent with a monomer. 

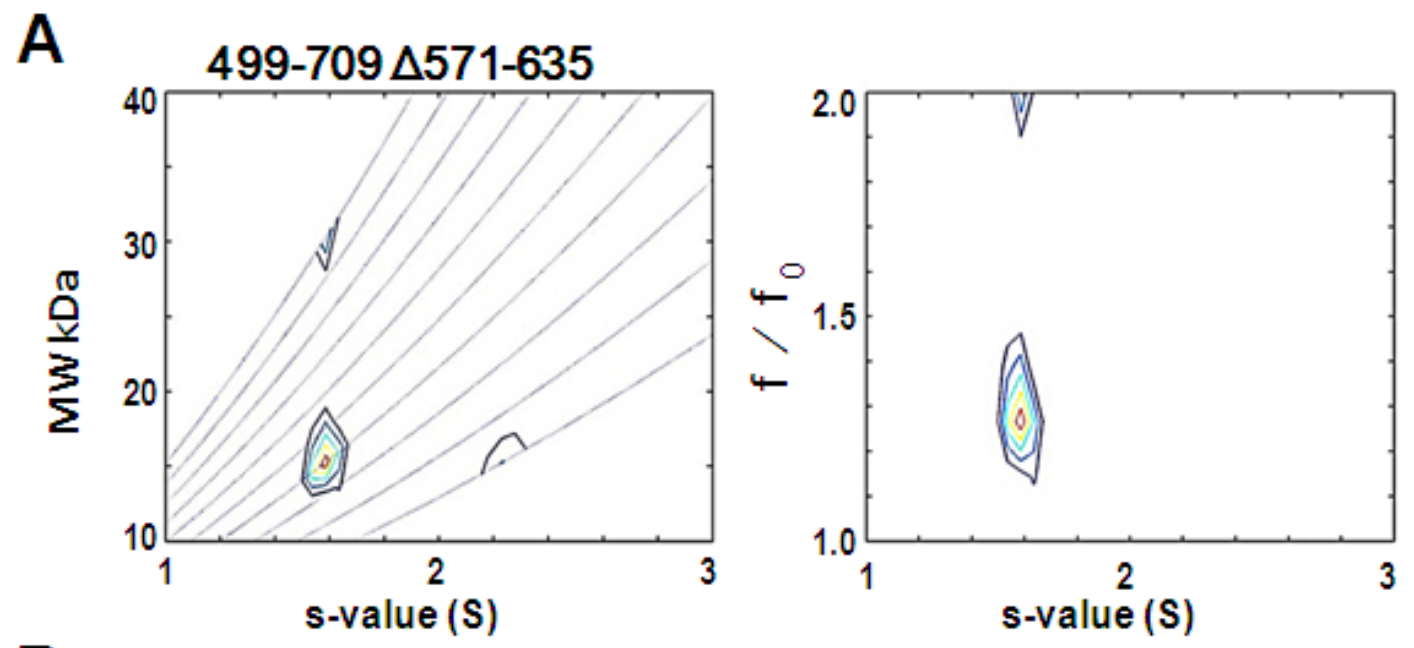

B
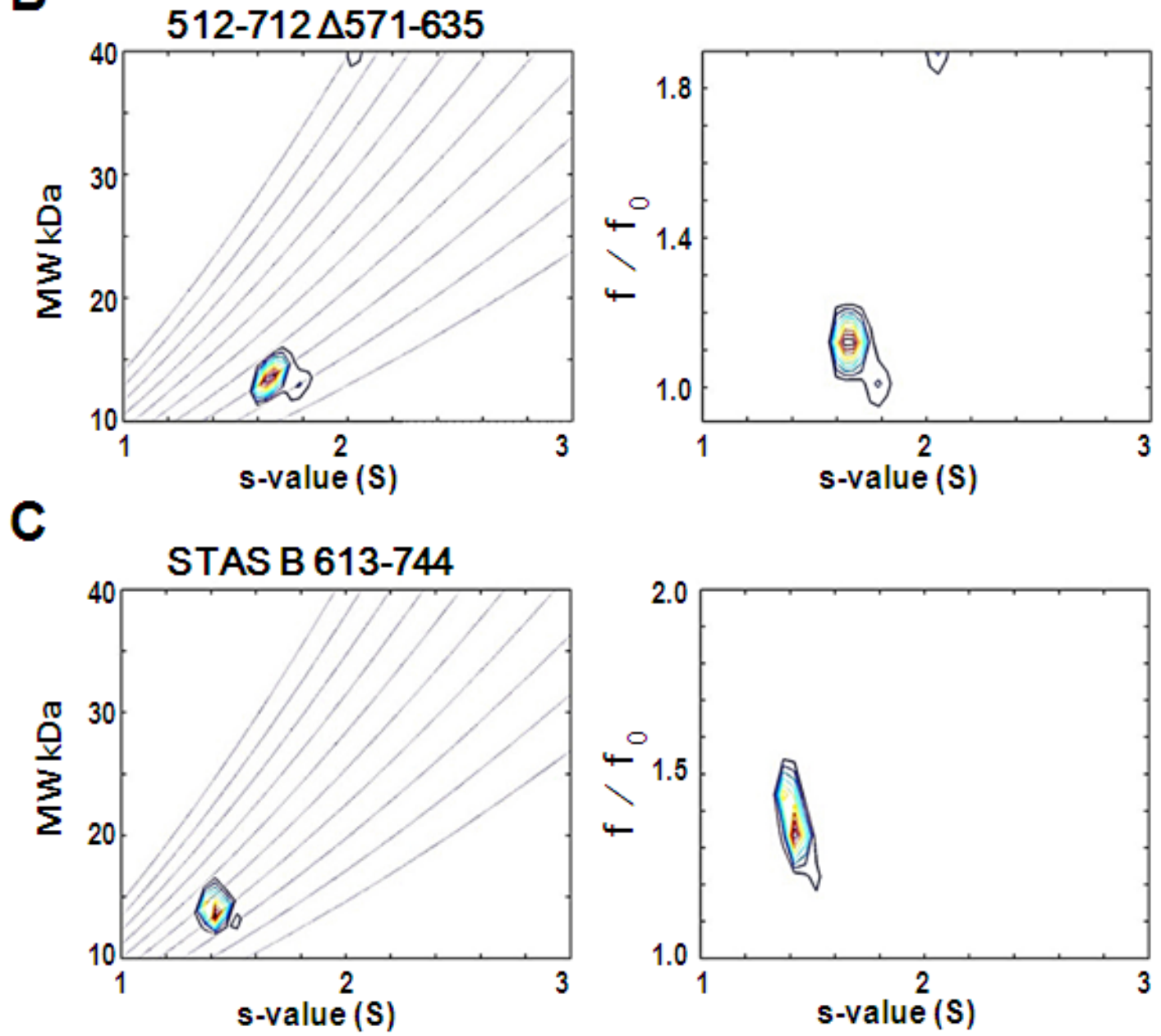

Figure 4-8 2D contour plots: 499-709 $\Delta 571-635,512-712 \Delta 571-635$, and STAS B (A) SV results for 499-709 $\Delta 571-635$. (B) SV results for 512-712 $\Delta 571-635$. (C) SV results for STAS B 613-744. All proteins exhibited a molecular mass consistent with a monomer. 
proteins are in reasonable agreement with their respective theoretical molar mass (in parentheses in Table 4-5).

In summary. several of the proteins displayed multiple peaks and exhibited varied hydrodynamic features in solution. In particular, the 499-744 $\Delta 571-635$ protein in saltfree buffer and 526-744 $\Delta 571-635$ protein showed these attributes. The $c\left(s, f / f_{0}\right)$ model allows a more reliable determination of the molar masses of sedimenting species with dissimilar $f / f_{0}$ values compared to the $c(s)$ distribution model, which can only accommodate a single $f / f_{0}$ value for all species. Thus, marked differences in shape were observed for individual C-terminus proteins in solution.

\subsection{C-terminus Is Folded but the Structure Difficult to Resolve}

In order to directly evaluate the C-terminus structure, NMR and circular dichroism were used to study the folding properties. Stability of the 512-712 $\Delta 571-635$ and 499-744 $\Delta 571-635$ proteins was tested by thermal denaturation. The $\alpha$-helical content for each protein was measured at $222 \mathrm{~nm}$ with regard to the increasing temperature. Both proteins appeared to be quite stable up to $55^{\circ} \mathrm{C}$ (see Figure 4-9). Each protein displayed a curve with a sigmoidal shape which is consistent to a simple transition between a folded and unfolded state. This data implies that interaction, over structural changes, between STAS A and STAS B underpinned the different conformations observed in the SV results. If STAS A or B transitioned between different folded states, then each conformation would have a separate free-energy value $(\Delta G)$. The resulting thermal denaturation curve would be more complex as the protein transitioned between more than one folded state to the final denatured state.

NMR spectra were collected for the 512-712 $\Delta 571-635,499-744 \Delta 571-635$, and STAS B 613-744 proteins (see Figure 4-10). All three proteins exhibited various degrees of folding. The 2D Trosy spectrum for 512-712 $\Delta 571-635$ indicates that the protein is well-folded protein based on the chemical shift dispersion of the nitrogen-proton crosspeaks. The 499-744 $\Delta 571-635$ protein also appears to be folded; however, it displayed features consistent with disorder or structural flexibility based on the different peak intensities. The peaks clustered around the spectra's center probably originated from the disordered residues. The spectra of STAS B 613-744 exhibit some degree of folding, but the crosspeaks cannot be resolved. The CD spectra for the proteins shown in Figure 4-11 indicates that each protein contains secondary structure with $\alpha$-helix and $\beta$-sheet elements. The data suggests that the proteins have similar structures to include the STAS B 613-744 protein. A minima around $200 \mathrm{~nm}$ was not observed, which is a signature for random coil. This indicates that disorder does not predominate over the folded areas in the protein based on the experimental conditions tested; however, this does not preclude its presence within the structure. 

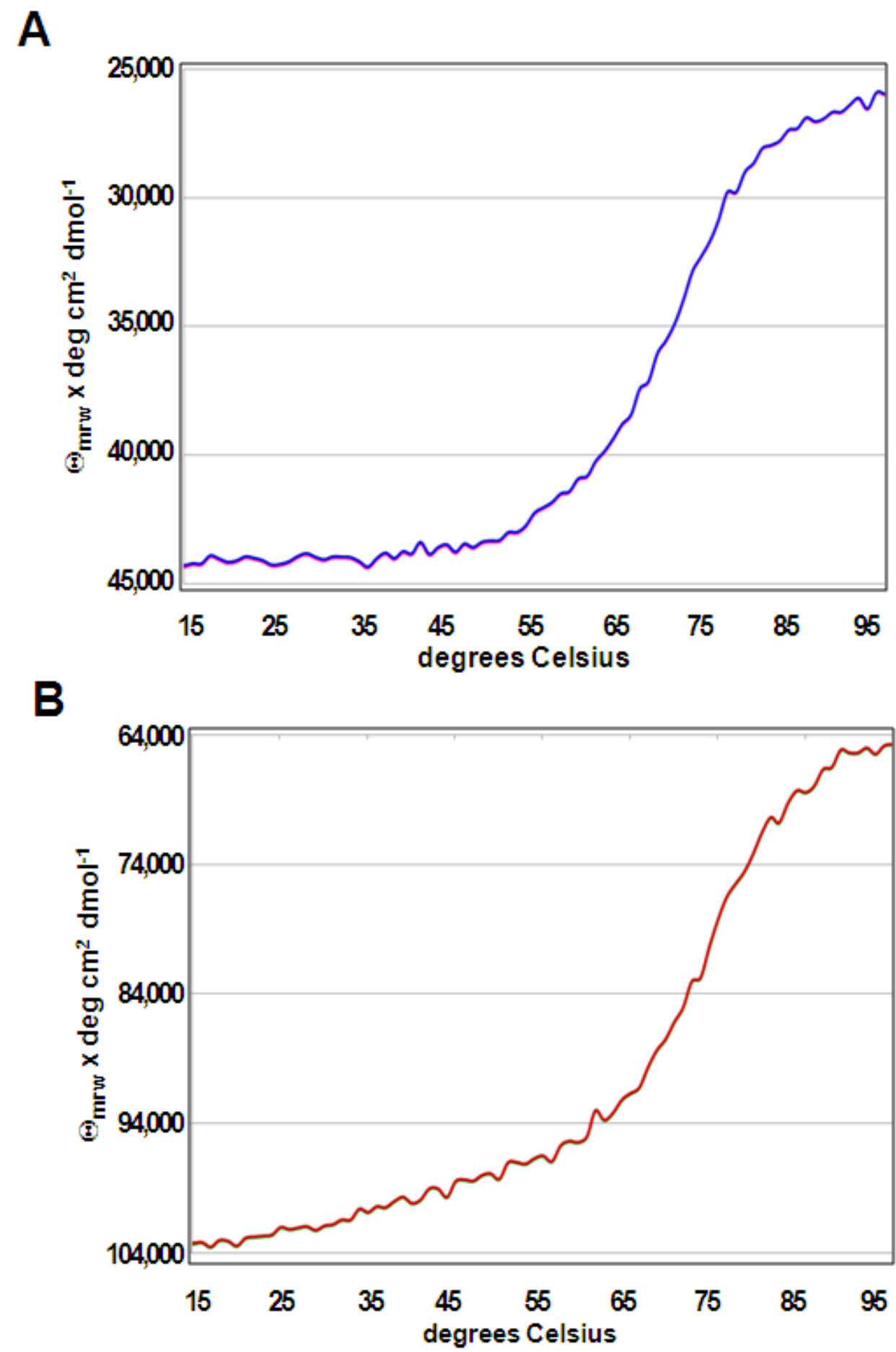

Figure 4-9 Thermal denaturation of 499-744 $\Delta 571-635$ and 512-712 $\Delta 571-635$

The curves for each protein shows the transition from a folded state $\left(\sim 15-55^{\circ} \mathrm{C}\right)$ to an unfolded one $\left(>55-95^{\circ} \mathrm{C}\right)$. Both proteins appear to be re relatively stable. $(A)$ Thermal denaturation curve for the 512-712 $\Delta 571-635$ protein. $(B)$ Thermal denaturation curve for the 499-744 $\Delta 571-635$ protein. 
A

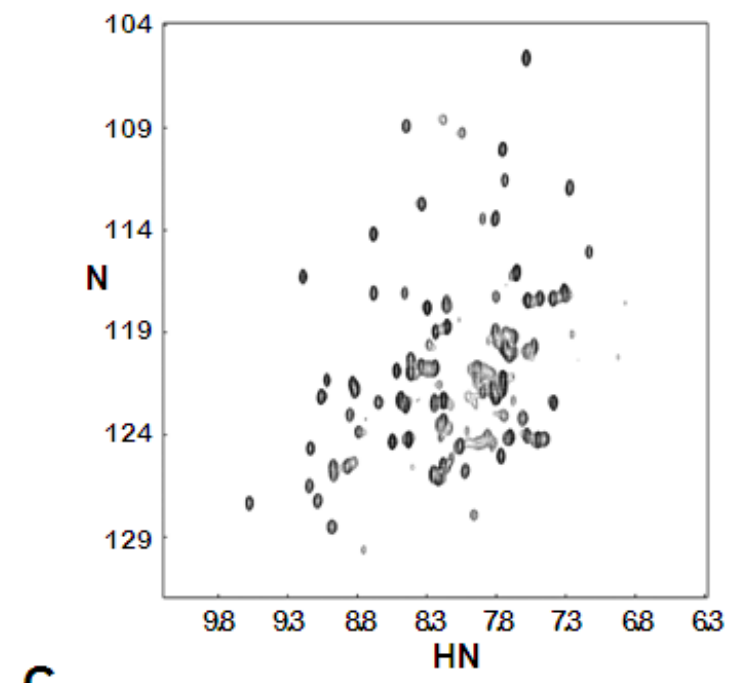

C

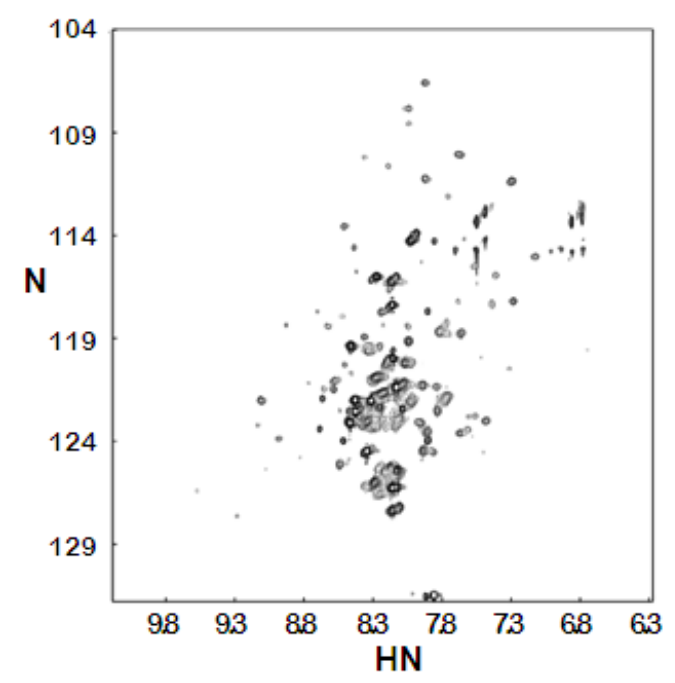

B

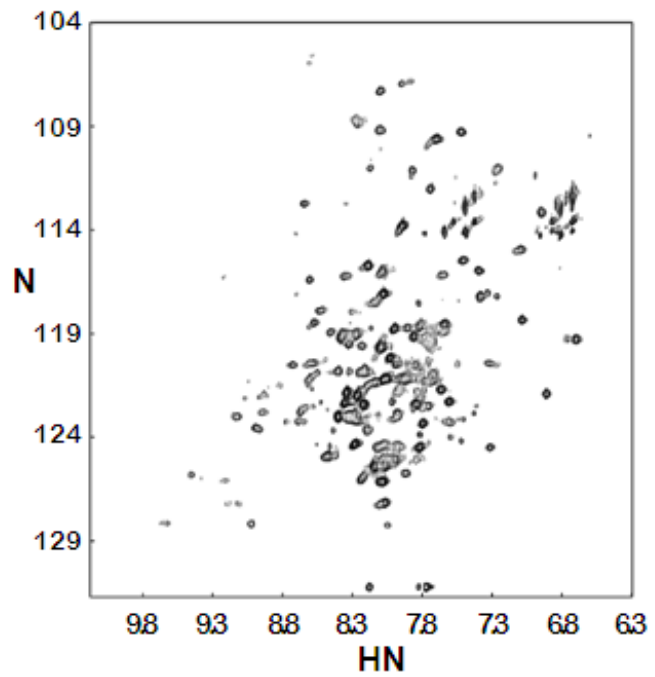

Figure 4-10 C-terminus is folded but structure cannot be resolved by NMR (A) Trosy spectra of ${ }^{15} \mathrm{~N}$-labeled 512-712 $\Delta 571-635$ appears consistent with a wellfolded protein; however, only 120 peaks (85\%) of 143 total can be counted. (B) NMR Trosy spectra of ${ }^{15} \mathrm{~N}$-labeled 499-744 $\Delta 571-635$ indicates that the protein is folded. Only 120 peaks out of 188 can be counted. The variable peak intensity suggests that the structure may not retain a static fold. (C) ${ }^{15} \mathrm{~N}$-labeled STAS B 613-744 appears to be folded based on the Trosy spectra, but the different peak intensities also hint that the structure may not retain a static fold. 
A

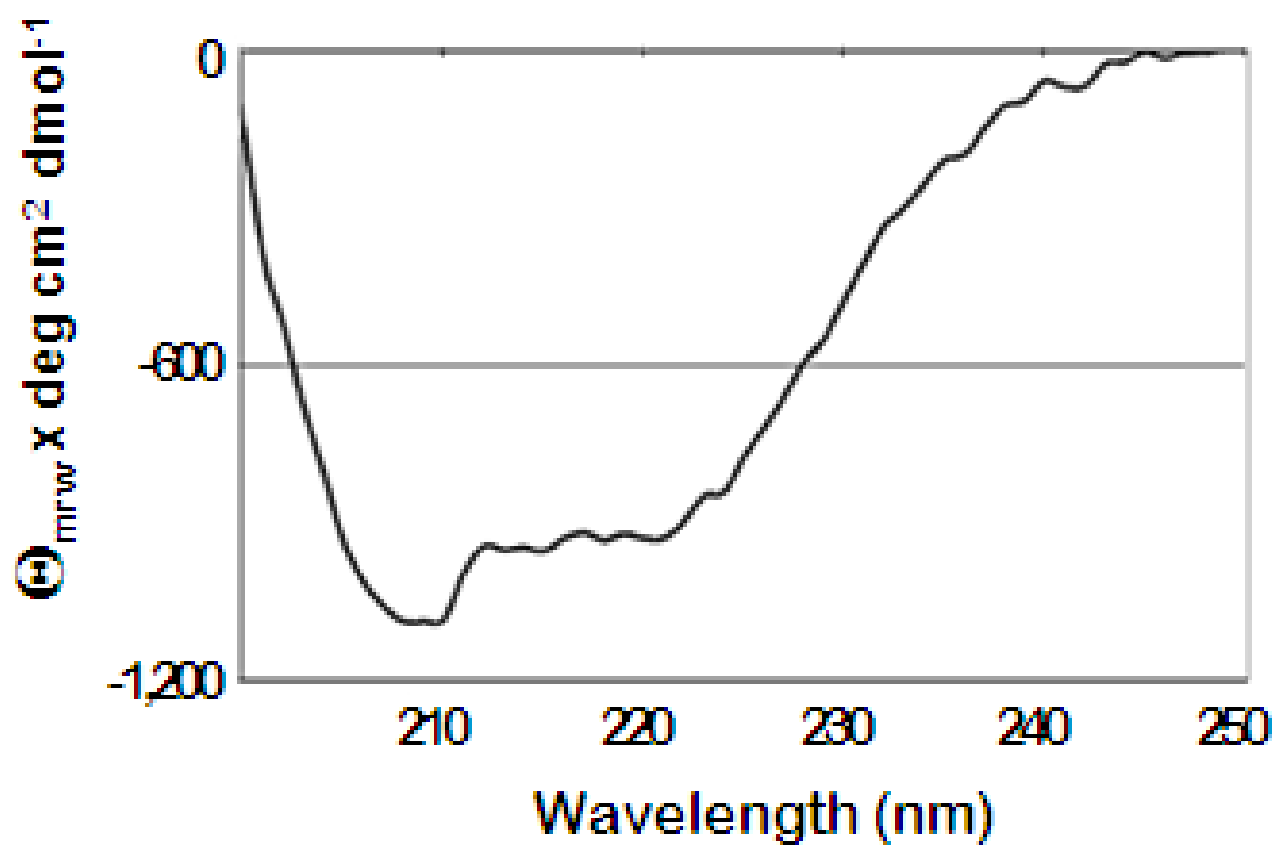

B

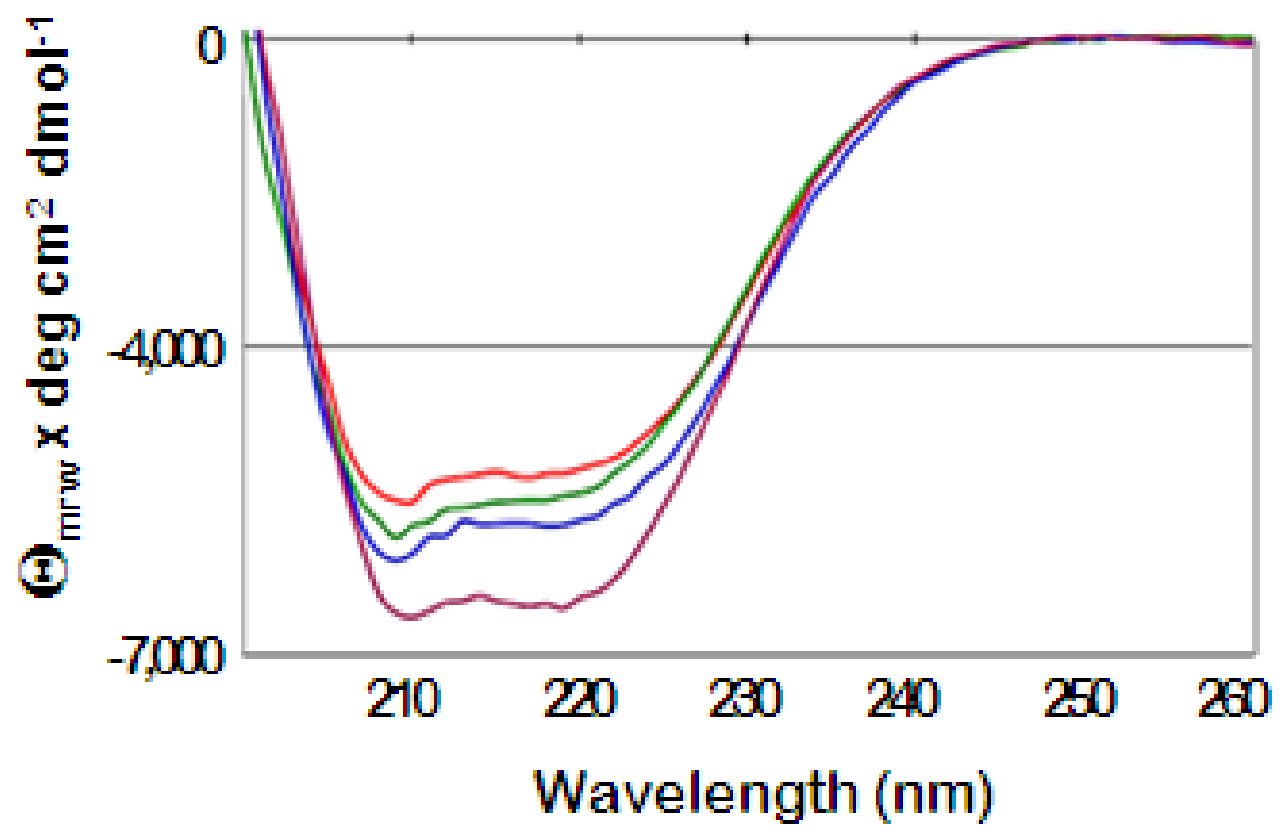

Figure 4-11 C-terminus proteins appear to have similar secondary structure (A) CD spectra shown for 499-744 (black trace). (B) CD spectra for proteins 512-712 $\Delta 571-635$ (red trace), 499-744 $\Delta 601-618$ (green trace), STAS B 613-744 (blue trace), and 499-744 $\Delta 571-635$ (purple trace). The spectra show that $\alpha$-helix, $\beta$-sheet, and random coil secondary structure are present. From this data, the sequence deletions did not appear to cause dramatic structural differences between the proteins. 


\subsection{Summary}

Structural aspects of the C-terminus were evaluated by deleting residues from sequence adjoining the predicted STAS A and STAS B. Trypsin and subtilisin results provided direct evidence showing that residues 571-635 of the loop is disordered. NMR and AUC represent independent means to explore structural aspects of a protein in solution. AUC data showed that several of the $\mathrm{C}$-terminus proteins exhibited different shapes in solution each with the same molecular mass. The inability to resolve all of the crosspeak resonances suggested that movement occurs in the structure. Overlap between these results led to the conclusion that the $\mathrm{C}$-terminus can adopt different conformations and showed that the $\mathrm{C}$-terminus did not have the propensity to oligomerize.

On the basis of the different deletions, the MW ranged from 14-27 kDa for the proteins characterized. Despite these differences, the minor axis values calculated from the AUC data fell between 2-3 nm for all proteins. The major axis spanned was rather elongated covering 7 to $15 \mathrm{~nm}$. These results imply three things. First, the proteins had a similar folding pattern, Second, the targeted deletions did not overtly disrupt the primary structure in the sequence, which supports the model proposed for STAS A (526-570) and STAS B (636-709). Third, the scale of major axis is from two to five times greater than the size of SpoIIaa determined from several structures [85, 86, 98]. Although the size for the C-terminus proteins represents the hydrodynamic value, the apparent difference implies that they are not folded the same as SpoIIaa STAS. From the data, it is hypothesized that the different conformations observed could result from interaction between STAS A and B with the loop serving as a tether. 


\section{Chapter 5 STAS A-B Interaction May Underlie C-terminus Conformations}

\subsection{Insights to the Different Conformations from Binding Kinetics}

The NMR spectra and behavior of the C-terminus in solution suggested that the protein could adopt various conformation. From the deletions made to the $\mathrm{C}$-terminus, this property appeared to be linked to the STAS A and STAS B. It was hypothesized that interaction between $\mathrm{A}$ and $\mathrm{B}$ could produce different conformations; therefore, the kinetics of the interaction could provide insight to underlying mechanism. To verify that binding occurs, SPR studies were performed using biotinylated STAS A peptides (see Table 5-1) and the STAS B 613-744 protein. The STAS B 613-744 protein served as the analyte in all tests which was flowed over synthetic A peptide covalently fixed to the sensor. Three different A peptides were tested. The first peptide consisted of residues 526-570 which corresponded exactly to the 'core' sequence predicted for the STAS A. The second peptide included residues 512-555 which started 15 residues before STAS A. Finally, the third peptide covered residues 534-577 beginning inside STAS A and ending seven residues into the loop.

The data gave clear evidence that reversible binding occurred between STAS B and the A peptides (see Figure 5-1); however, a small deviation was observed at the highest concentration $(150 \mu \mathrm{M})$ of STAS B. For the 526-570 A peptide, a moderate binding constant was observed $\left(\mathrm{K}_{\mathrm{d}}\right)$ of $45.8 \mu \mathrm{M}$ with a slow on-rate $\left(\mathrm{k}_{\mathrm{a}}\right)$ of $2,120 \mathrm{M}^{-1} \mathrm{~s}^{-1}$ and a moderately slow off-rate $\left(\mathrm{k}_{\mathrm{d}}\right)$ of $0.0972 \mathrm{~s}^{-1}$ (see Figure 5-1A and Table 5-2). The binding constant for the 512-555 A peptide yielded similar results. In contrast, STAS B 613-744 was unable to bind the 534-577 A peptide. As a second experiment, purified STAS B 613-744 from Mono Q (anion exchange) purification was isolated into two fraction pools based on low-salt $(\sim 200 \mathrm{mM} \mathrm{NaCl})$ or high-salt $(\sim 400 \mathrm{mM} \mathrm{NaCl})$ elution. Both fraction pools were prepared in $25 \mathrm{mM}$ potassium phosphate $\mathrm{pH} 7.0,150 \mathrm{mM} \mathrm{NaCl}$, $5 \mathrm{mM}$ DTT, and $0.005 \%$ Tween 20 . Binding was measured for concentration ranges of 1.9 to $150 \mu \mathrm{M}$. The $\mathrm{K}_{\mathrm{D}}$ of the low-salt was $29.5 \mu \mathrm{M}$, while the $\mathrm{K}_{\mathrm{D}}$ of the high-salt was $51.5 \mu \mathrm{M}$ (see Table 5-3). The different binding affinities suggest that each 'salt' pool may have contained different folding populations (conformations) of the STAS B 613744.

\subsection{Yeast Two-Hybrid Shows Binding Can Occur in the Cell}

To determine whether the binding event can occur within the cell, a yeast twohybrid assay was used. The 'activating domain' (AD) consisted of residues 499-564 which represented the A-half of the STAS. Residues 639-744 represented the B-part and served as the 'binding domain' (BD). The following (+) and (-) controls were performed. The plasmid expressing the A part fused to the Gal4 transcription activation domain did not activate the reporter genes (Figure 5-2D). Co-expression with the Gal4 DNA-binding domain only also did activate the reporters (Figure 5-2B). Likewise, the plasmid expressing the B-part fused to the Gal4 DNA-binding domain did not activate the 
Table 5-1 Sequence of biotinylated peptides used in SPR studies

\begin{tabular}{ll} 
Residues & Peptide sequence \\
\hline $512-555$ & GQLPDTDVYIDIDAYEEVKEIPGIKIFQINAPIYYANSDLYSSA \\
$526-570$ & YEEVKEIPGIKIFQINAPIYYANSDLYSSALKRKTGVNPALIMGA \\
$534-577$ & GIKIFQINAPIYYANSDLYSSALKRKTGVNPALIMGARRKAMRK \\
\hline
\end{tabular}


A

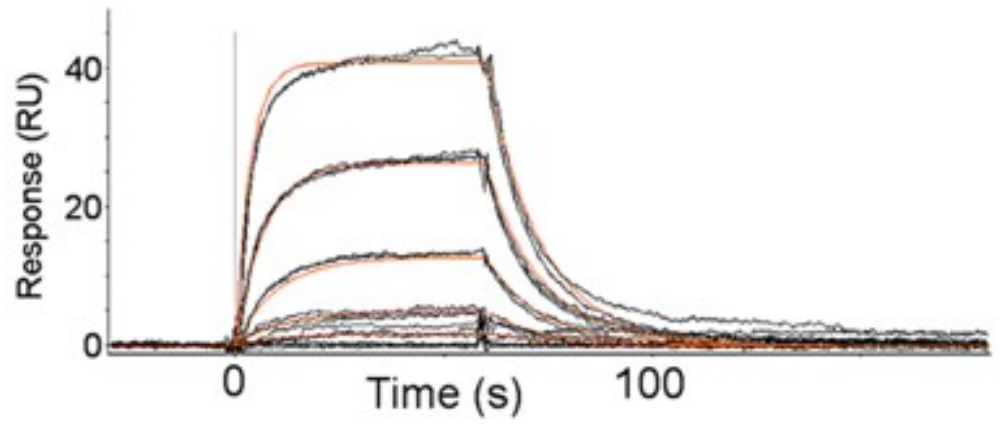

B

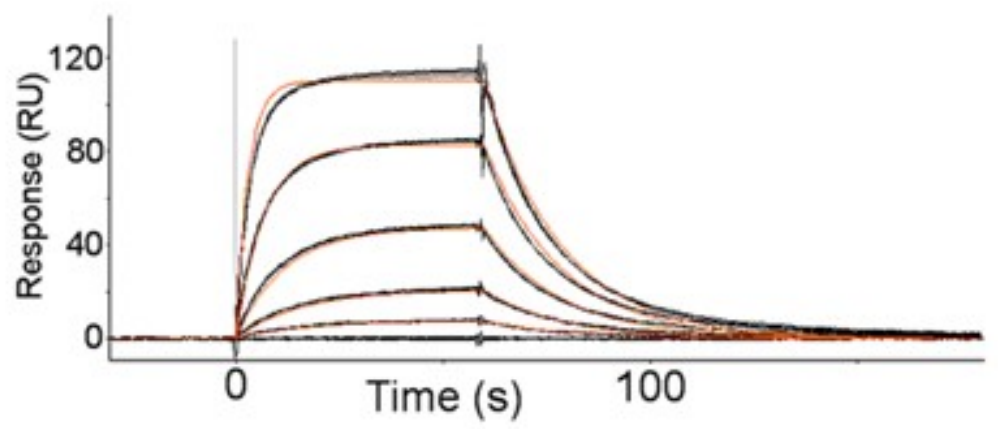

C

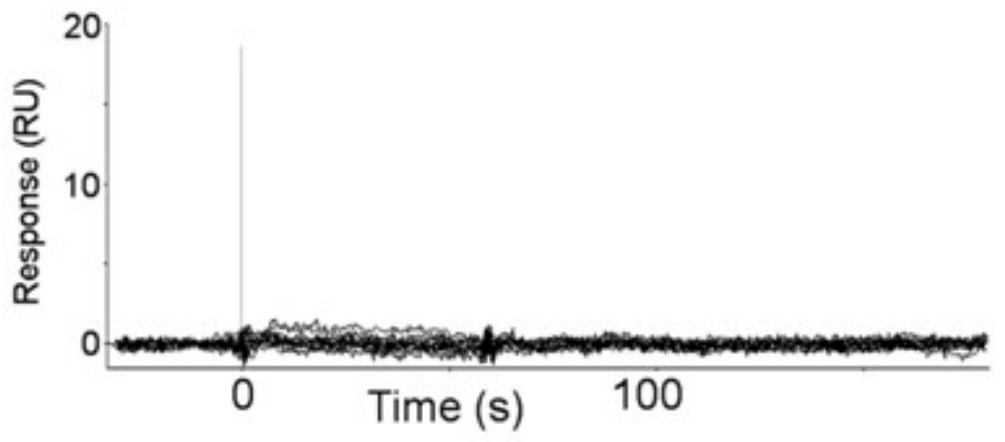

Figure 5-1 Surface plasmon resonance interaction between STAS A and B Each panel composed of the sensorgrams overlaid with kinetic analysis fit $(A)$ Sensorgram of biotinylated STAS A 526-570 peptide binding to B 613-744. (B) Biotinylated STAS A 512-555 peptide binding to B 613-744. (C) Biotinylated STAS A 534-577 peptide binding to STAS B 613-744. 
Table 5-2 STAS A peptide and STAS B 613-744 SPR reversible binding data

\begin{tabular}{lllllll}
\hline Peptide & $\begin{array}{l}\boldsymbol{k}_{\mathrm{a}} \times \mathbf{1 0}^{\mathbf{3}} \\
\left(\mathbf{M}^{-1} \mathbf{s}^{-\mathbf{1}}\right)\end{array}$ & $\begin{array}{l}\boldsymbol{k}_{\mathrm{d}} \times \mathbf{1 0}^{-\mathbf{2}} \\
\left.\mathbf{( s}^{-\mathbf{1}}\right)\end{array}$ & $\begin{array}{l}\mathbf{K}_{\mathbf{D}} \\
(\boldsymbol{\mu M})\end{array}$ & $\begin{array}{l}\mathbf{R}_{\max } \\
(\mathbf{R U})\end{array}$ & $\begin{array}{l}\mathbf{K}_{\mathbf{D}} \\
(\mathbf{e q})(\boldsymbol{\mu M})\end{array}$ & $\begin{array}{l}\mathbf{R}_{\max } \\
(\mathbf{e q})(\mathbf{R U})\end{array}$ \\
\hline $\mathbf{5 2 6 - 5 7 0}$ & 2.12 & 9.72 & 45.8 & 45.7 & 46.7 & 47.7 \\
& \pm 0.01 & \pm 0.04 & \pm 0.20 & \pm 0.10 & \pm 0.70 & \pm 0.30 \\
$\mathbf{5 1 2 - 5 5 5}$ & 2.72 & 5.47 & 20.1 & 132 & 20.8 & 137 \\
& \pm 0.01 & \pm 0.02 & \pm 0.10 & \pm 1.00 & \pm 0.10 & \pm 1.00 \\
$\mathbf{5 3 4 - 5 7 7}$ & - & - & - & - & - & - \\
\hline
\end{tabular}


Table 5-3 A 512-555 peptide and STAS B 613-744 Mono $Q$ fraction SPR data

\begin{tabular}{lllllll}
\hline $\begin{array}{l}\text { STAS B } \\
\mathbf{6 1 3 - 7 4 4}\end{array}$ & $\begin{array}{l}\boldsymbol{k}_{\mathrm{a}} \times \mathbf{1 0}^{\mathbf{3}} \\
\left(\mathbf{M}^{-1} \mathbf{s}^{-\mathbf{1}}\right)\end{array}$ & $\begin{array}{l}\boldsymbol{k}_{\mathrm{d}} \times \mathbf{1 0}^{-2} \\
\left(\mathbf{s}^{-\mathbf{1}}\right)\end{array}$ & $\begin{array}{l}\mathbf{K}_{\mathbf{D}} \\
(\boldsymbol{\mu M})\end{array}$ & $\begin{array}{l}\mathbf{R}_{\max } \\
(\mathbf{R U})\end{array}$ & $\begin{array}{l}\mathbf{K}_{\mathbf{D}} \\
(\mathbf{( e q})(\boldsymbol{\mu M})\end{array}$ & $\begin{array}{l}\mathbf{R}_{\max } \\
(\mathbf{e q})(\mathbf{R U})\end{array}$ \\
\hline $\mathrm{Q} \# 12-15$ & $2.57 \pm 0.01$ & $7.58 \pm 0.03$ & $29.5 \pm 0.10$ & $57.0 \pm 0.1$ & $30.7 \pm 0.4$ & $59.1 \pm 0.2$ \\
$\mathrm{Q} \# 15-18$ & $1.58 \pm 0.02$ & $8.13 \pm 0.03$ & $51.5 \pm 0.3$ & $41.3 \pm 0.1$ & $53.2 \pm 0.8$ & $42.8 \pm 0.3$ \\
\hline
\end{tabular}



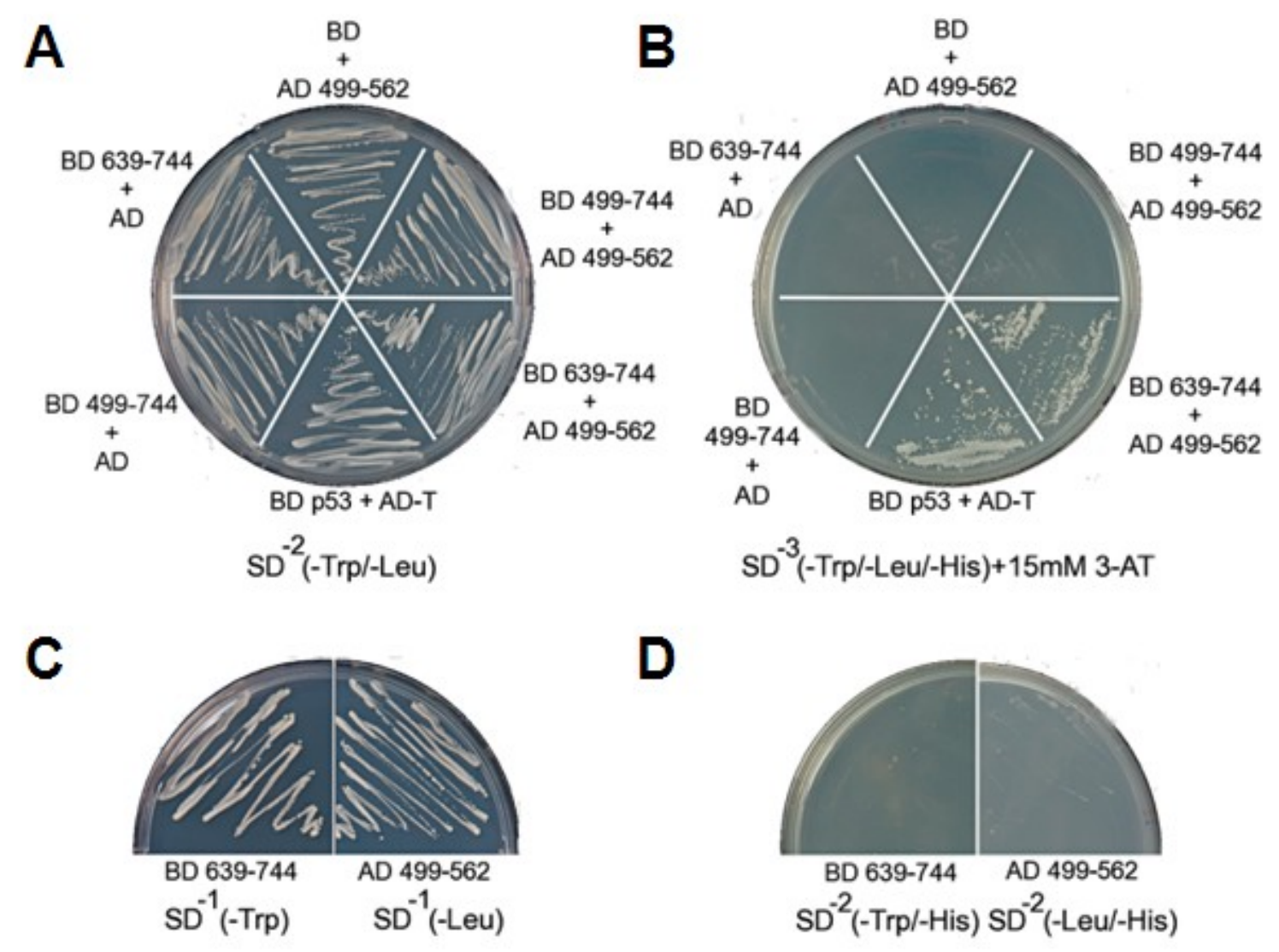

Figure 5-2 Yeast two-hybrid showing interaction between 499-564 and 639-744 Positive interaction activated transcription of the His reporter gene allowing growth on His-, Leu-, Trp- media in the presence of the metabolic inhibitor, $15 \mathrm{mM}$ 3-AT (3-amino1, 2, 4-triazole). The GAL4 DNA binding domain and transcriptional activation domain are indicated by $\mathrm{BD}$ and $\mathrm{AD}$, respectively. (A) The control plate lacks tryptophan and leucine, and indicates co-transformation of the yeast cells with both a pGBK and a pGAD plasmid. (B) Activated transcription of histidine reporter genes by 499-564/639-744 interaction or $\mathrm{p} 53 / \mathrm{SV} 40$ large T-antigen (positive control) interaction activated transcription of reporter genes as indicated by growth on His- Leu- Trp- $15 \mathrm{mM}$ 3-AT synthetic drop (SD) medium and blue color on $\beta$-galactosidase assay (data not shown). Note that co-expression of 499-744 and 639-744 in yeast failed to activate the HIS3 reporter gene. $(C)$ The control plate lacks either tryptophan or leucine, and indicates transformation of the yeast cells with either a pGBK or a pGAD plasmid. (D) The plasmid expressing either 639-744 fused to the Gal4 DNA-binding domain alone or 499564 fused to the Gal4 transcription activation domain did not activate the reporter genes. This figure has been adapted for presentation. 
reporter alone (Figure 5-2D). Furthermore, co-expression with the plasmid only expressing the Gal4 transcription activation domain did not produce activation (Figure 5-2B). Upon expression of both the A and B-parts, activation of the His-reporter occurred (Figure 5-2B). These results supported interaction between the A and B-parts; however, they do not distinguish whether intermolecular or intramolecular interaction occurred in the yeast cells. To test the nature of the interaction, the A-part, fused to the Gal4 transcription activation domain, was co-expressed with the entire $\mathrm{C}$-terminus, residues 499-744. The C-terminus was fused to the Gal4 DNA-binding domain. As shown, the yeast failed to activate the His reporter gene (Figure 5-2B). From these observations, it was concluded that interaction for the A and B-parts does not occur between separate molecules. Therefore, the $\mathrm{r}$ results support an intramolecular binding event for the $\mathrm{A}$ and B-parts in yeast cells.

\subsection{Summary}

Reversible binding occurs between peptides for STAS A and recombinant protein for STAS B 613-744. Both the reversible nature of the interaction and the modest affinity suggest that similar events could occur within the $\mathrm{C}$-terminus 499-744 producing the different conformations observed in chapter 4 . Yeast two-hybrid assay also demonstrated similar binding which provided evidence showing the specificity of the interaction. Binding did not occur when the entire $\mathrm{C}$-terminus was used in the yeast two-hybrid. This result indicated that the interaction is predominantly intramolecular and not intermolecular. 


\section{Chapter 6 Discussion}

\subsection{Synopsis}

My efforts focused on the structural and biochemical characterization of prestin's C-terminus. From the data, it was possible to develop a structural map of the STAS domain within the C-terminus. These insights were used to investigate the functional role of the C-terminus in full length prestin. The disordered loop, around 65-residues in length, displays traits consistent with function as part of prestin's voltage sensor [51, 52]. Collectively, the data from all studies led to the following four major conclusions. It is unlikely that prestin's C-terminus has any role tied to prestin-prestin interaction. The Cterminus does appear to have biochemical properties that allow it to change conformation. The STAS domain appears to consist of two subdomains that can interact in a reversible manner.

\subsection{C-terminus Unlikely to Have a Role Supporting Prestin-prestin Interaction}

A previous study demonstrated that recombinant protein of the prestin C-terminal STAS domain adopted multiple oligomeric states in solution [97]. In contrast, our analytical ultracentrifugation and static light scattering data show that all C-terminal STAS domains exist as monomers in solution. In addition we determined from our SV analysis that these C-terminal STAS domains adopt various molecular shapes, from globular to elongated. The oligomeric states observed in the previous study are likely a misinterpretation of the data based on the techniques used (size-exclusion chromatography and dynamic light scattering). Size-exclusion chromatography does not always provide the correct protein mass in solution because of differences in molecular shape [125]. In particular, elongated shapes simply do not fit well into the rigid pores of the gel matrix which are designed to accommodate more globular proteins solely based on their mass. The $\mathrm{C}$-terminus proteins studied here consistently eluted from a analytical size-exclusion column over a range of MW ranging from 100-600 kDa relative to the standards used (data not shown). The column had been calibrated using standards from two different sources. Dynamic light scattering, while very robust when analyzing monodisperse samples, is not as reliable for samples that can adopt multiple conformations [126]. In contrast, classic static light scattering reliably identified the monomeric form of C-terminal STAS domains in this study since it measures the absolute molar mass of medium-size proteins independent of shape. SV, which is a superior method for determining the size-and-shape distributions of proteins in solution [122], also showed that the C-terminal STAS domain mutants were monomers that adopted different shapes.

Given that the C-terminus proteins only existed as monomers in solution and that this state overlapped with the presence of different conformations, it is conceivable that these properties could have physiological in full-length prestin. Therefore, it is proposed that there is an as-yet unidentified event or stimulus that causes the C-terminus to shift 
the equilibrium of its conformational state so that the C-terminus preferentially becomes either elongated or more globular under certain conditions, thus modulating prestin's conformational state in the membrane and electromotility. This model contrasts strikingly with other previously proposed models that maintain the oligomerization of the Cterminus.

Tethered subdomains that interact weakly and reversibly could result in various conformational states in dynamic equilibrium. Because the interaction is relatively weak, and because intramolecular interactions are generally favored at increased apparent protein concentrations, the probability of high-order oligomeric states seems remote. The shape of the SPR curves also indicates a lack of oligomeric and aggregated material in the reaction solutions, except where they deviate from 1:1 Langmuir binding at the highest analyte concentrations. These conclusions are supported by the AUC and light scattering results where only monomeric forms were observed for all C-terminus constructs. It seems that intra-molecular association of prestin domains may give rise to a different relative domain orientation and arrangement that changes the overall shape of the molecule (i.e. more globular or more extended) and perturbs a given promoter's function. Therefore, molecular shape could control the function of prestin in hearing.

\subsection{C-terminus Appears to Adopt Multiple Conformations in Solution}

Together, the results indicate that the STAS domain in the C-terminus is composed of two subunits which exhibit reversible interaction in solution. A disordered loop region, 65 residues in length, partitions the STAS into distinct A and B subdomains. The nature of the interaction coupled to the loop's flexibility causes the protein to adopt different molecular shapes in solution. It is reasonable to consider that bound and unbound states produce display different folding properties. The bound state would have a more compact, globular shape, while the unbound state would have a more extended shape. There is no evidence of oligomerization taking place under the conditions tested.

Both AUC and NMR results indicate that the C-terminus may adopt various shapes (conformations) in solution. From this, it is proposed that this occurs as a consequence of the reversible interactions between STAS A and B with the loop serving as a tether between the two areas. Such behavior would result in protein mixtures with either dissimilar frictional ratios or a wide range of frictional ratios. This interaction was confirmed by the SPR data, which showed that the two subdomains could indeed interact, albeit relatively weakly. The relatively slow association rate for STAS B 613-744 suggests that it undergoes conformational adaptation before binding. A reasonable assumption would be that binding requires a conformation of STAS B 613-744 that exists in relatively low abundance in the total STAS B population. This behavior is consistent with the slow on-rate observed for ligand binding to the estrogen receptor [127]. For the C-terminus, the binding kinetics don't give suggestion that the STAS A and B would interface more robustly in solution allowing only one conformation. It's possible that the observed behaviors in solution resulted solely from the disordered sequence in the protein. The random nature of disorder on a protein's surface would certainly change the 
hydrodynamic properties of the protein. This could account for some of the solution behavior. At the same time, the sedimentation behavior should be consistent between proteins considering that the A and B remained the same. However, that is not what the data shows.

By default, the 499-744 protein had the largest 'disorder' content to include residues 571-635 and 710-735. The protein exhibited a range of sedimentation coefficients which could be attributed to entropic movement. The other proteins also exhibited different conformations, but the sedimentation values could be resolved. As a comparison to 499-744, close to half the STAS B 613-744 protein's sequence consisted of sequence that was disordered based on prediction and experiments. NMR studies showed that it did not fold well based on unresolved spectra. If disorder was a dominant factor, then one could expect to see a sedimentation profile similar to the one for 499744. That did not occur. The data showed a single, monomeric protein which would imply that both subdomains are needed to manifest as multiple conformations.

Additionally, the folding properties of the different proteins hinted that reversible binding produced the conformations, based on how the size, shape, and folding changed relative to the sequence area deleted. Removal of the disorder from the sequence should have produced more uniformity between these properties. That's not what occurred. The differences ranged from more compact, globular to ellipsoid, and protracted. Proteins that maintained residues 571-635 or 710-744 showed more than one shape and folding that produced an elongated, prolate ellipsoid form. Deletion of both these areas yielded more distinct shape populations and more compact, globular folds.

Because the loop forms a tether between the STAS subdomains, any factor that perturbs the loop's structural state will change the likelihood of intramolecular binding between the two subdomains, and thereby affect the overall conformation and molecular shape. AUC allows direct measurement of hydrodynamic properties that reflect the average global conformation of a protein in solution. The s-values and frictional ratios for the C-terminus proteins (499-744, 526-744 polar, 499-744 $\Delta 601-618$, 499-744 $\Delta 571-635$, and 526-744 $\Delta 571-635$ ) showed that they have an extended shape and are thus presumably relatively flexible. When a protein is flexible, it samples conformational space to adopt multiple conformations. For instance, an intrinsically unstructured protein is of central importance in the multistep phosphorylation/ubiquitination signal transduction pathway that regulates cell division [128]. Protein flexibility has been shown to be critical in other signaling pathways as well. For example, the ability to undergo structural rearrangements in response to allosteric signals is critical for remodeling of the actin cytoskeleton [129]. Based on the proposed model with the loop connecting STAS A to STAS B, it is possible that this architecture could support the voltage sensor function or support a connections to the cortical lattice. Recent data supports this possibility. It was shown that prestin's C-terminus, via an intermediary protein, interacts with the cortical lattice. It has been suggested that intrinsic protein flexibility could allow the transduction of multiple signals within a single protein structure due to the ability of different motifs within one polypeptide to "communicate" with each other. Clearly, the STAS A and B domains interact in a reversible manner with each other, which may cause the whole protein to adopt different molecular shapes on a 
physiological time-scale. The observation that the 499-744 $\Delta 571-635$ protein adopts a more elongated molecular shape in a reduced salt environment hints that it is inherently capable of adopting various molecular conformations. Also of note, prestin's C-terminus only contains one cysteine. Thus, its folding is not locked down by disulfides. It is then reasonable to propose that prestin's C-terminus changes molecular shape on a physiological time-scale, and that this mechanism could serve to propagate structural changes onto the cortical lattice.

\subsection{Prestin's STAS Composed of Two Subdomains}

To date, a structure of the eukaryotic STAS domain is not available. The 3D structure for the Bacillus SpoIIaa STAS has been solved. Thus, it is the primary means of structural insight for eukaryotic proteins that contain a STAS domain. The STAS sequence only exhibits $20-30 \%$ similarity between prokaryotes and eukaryotes. Reference to the STAS in Prestin literature is not consistent regarding the domain boundaries and length $[68-70,97]$. These issues were addressed in the current study. Characterization in solution indicated that residues 526-570 and 636-709, STAS A and B, form the primary structure with 571-635 existing as a disordered loop. Two experiments indicated that the loop could extend over a larger sequence, which would shift the structure boundaries proposed for A and B. First, the SPR results showed that the 512-555 peptide bound tighter over the 526-570 peptide. Second, the 512-712 $\Delta 571-635$ protein folded better than the 499-744 $\Delta 571-635$ protein tested with NMR. SV data highlighted the fact that the conformations manifested in multiple proteins. It looked as if the conformations transitioned between two relative states, compact and elongated. Unfortunately, it remains unclear how the transition occurred and what underlying folding changes supported the activity. The SPR data appears to back up the idea that the conformations derive from mutable interaction between STAS A and B. The slow on-rate appears consistent with a large conformational event preceding the binding. Additionally, the relatively modest KD values and reversibility show that the process is not static. The strength of the combined results suggests that the conformational behavior is an innate property of prestin's C-terminus. It seems possible that the conformational ability could underlie prestin's gating.

The different conformations observed also supports the hypothesis that prestin's STAS domain may not fold the same as SpoIIaa. The Bacillus SpoIIaa protein folds with a $\alpha / \beta$ two-layered sandwich arrangement. Alignment of SpoIIaa and C-terminus sequences shows poor conservation, especially in the termini. This suggests that the structures may differ and that predictions may not be reliable. Over time, residue insertions, mutations, and deletions gradually distort the original structure which can produce different functional attributes [130]. Prediction of prestin's C-terminus yields a model with SpoIIaa/STAS folding; however, non-overlapping areas of sequence (approximately 110 residues in lengths) can be used as the input to produce similar 3Dstructures (data not shown). This suggests that the $\mathrm{C}$-terminus folding may only loosely follow the SpoIIaa folding pattern. Furthermore, the $\alpha / \beta$ (sheet/helix) repeats in SpoIIaa appear to match-up to more than one area in the $\mathrm{C}$-terminus from structure prediction. As 
such, proper alignment of the SpoIIaa and C-terminus folds does not provide a clear indication of the STAS boundaries in the C-terminus. Finally, the experimental results presented indicate that two disordered regions exist in the C-terminus. The loop area, residues 571-635, is not part of the STAS domain based on sequence alignment. Thus, it divides the folding and appear to create two STAS subdomains which could facilitate novel structure and functionality. To that end, the STAS domain may have evolved a modular function. The prokaryotic domain largely exists as a single protein. In eukaryotes, it is often a component of a larger protein.

\subsection{C-terminus Loop Contributes to Prestin's Voltage Sensor}

Limited proteolysis data supported the idea that 571-635 lacked any fixed structure based on susceptibility to trypsin [131]. Incidental to the full-length prestin, deletion of the loop did not show any obvious structural abnormality based on membrane localization and exhibition of NLC. This implies that this sequence does not fulfill any static role tied to membrane structure. Functionally, protein disorder is tied to the transition between unstructured and structured states due to physiological changes [132]. The reverse transition also produces function [133]. Both mechanisms have been specifically linked to voltage-gated channels $[134,135]$. By extension, the disorder in the C-terminal loop may work in a similar manner as part of prestin's voltage sensor. Deletion of residues 571-635 from the C-terminus of full-length prestin increased the $\alpha$ value, which describe the voltage dependence of the charge transfer. Prestin's membrane activity is described by two-state Boltzmann mechanics. This function provides a statistical probability of prestin's conformational state, relative to the location of charge intrinsic to prestin within the membrane's electrical field. Thus, the larger $\alpha$-value indicates that prestin's conformational ability is retarded somehow. This result was surprising considering that properties associated with voltage gating in a protein are intrinsic to the membrane. From this result, it was concluded that the C-terminus must associate intimately with the membrane in order to detect changes within the membrane's electrical field. It is possible that this interaction could occur via several residues in the transmembrane domain, based on mutational studies that caused a similar outcome for the $\alpha$-value. Fourteen amino acids in prestin's sequence are linked to the voltage sensor. Four are negatively charged residues including E207, E280, D370, and D457. Eight residues area positively charged including R130, R211, K255, K276, K359, K364, K449, and R463. E211Q, S238, 364, 449, 457, and 463 had similar effects [53, 136].

In principal, the voltage sensor and gating activity are phenomenon confined to the membrane's electrical field [52]; however, at least two STAS domains have a gating function associated with their cytosolic location. Binding of the STAS domain from either SLC26A3 or SLC26A6 to the CFTR R-domain facilitates the gating of both proteins [137]. CFTR gating relies upon the disorder of the R-domain in solution [138]. Disorder often coincides with sequence that is variable in length and composition among related proteins [116] The loop is quite variable between SLC26 proteins and even in prestin species [43] (see Figure 6-1). It is plausible to think that the loop's voltage sensor function requires all 70 residues between 571-635. That would explain why earlier 


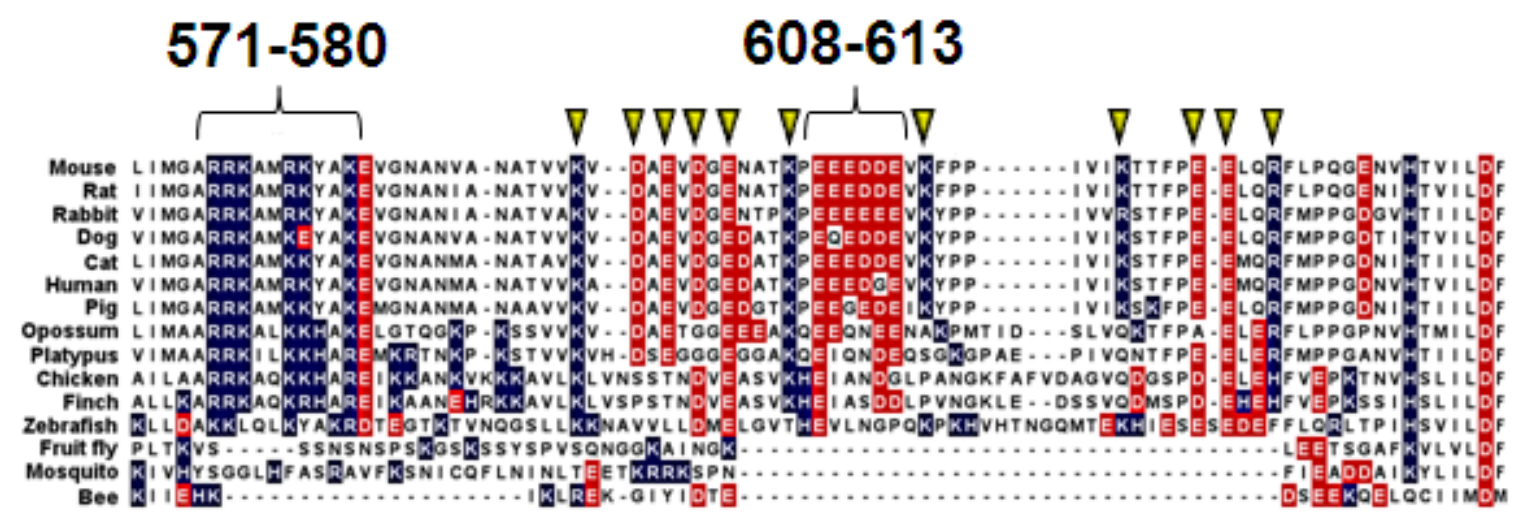

Figure 6-1 Diversity of charged residues in the loop of prestin species

Mouse prestin (top sequence) starts at residue 566 and goes to residue 646. Nonmammalian prestin's constitute the bottom four sequences. Two avian prestin sequences are above the non-mammalian sequences. The brackets denote residues that were mutated in other prestin studies, but did not produce data supporting a voltage sensor relationship. The yellow inverted triangles point out charged residues that have not been studied. Residues highlighted in dark blue are positively charged, while those in red are negatively charged. 
studies did not observe a similar effects to the $\alpha$-value or slope-factor, which describe prestin's voltage dependence $[53,70]$. Point mutations were made to the charged residues within much smaller areas. Thus, the data presented here suggests that the entire loop is required to contribute to the voltage sensor function.

A recent study ascribed several charged residues, close to the intracellular surface, as belonging to prestin's voltage sensor [136]. Their data, although relevant, could not explain the inconsistency it produced with data for the SLC26A6 protein and prestin orthologues. Both the SLC26A6 and orthologues contain the same residues identified to contribute to the voltage sensor in mammalian prestin. However, the SLC26A6 does not have NLC, while the orthologues have NLC with a reduced voltage-dependence and shifted membrane potential. Non-mammalian prestin also does not have electromotility [139]. The loop voltage sensor, residues 571-635, overlaps with one of the least conserved parts of prestin and all of the SLC26 proteins [43, 54, 140]. The sequence appears variable especially concerning the overall charge composition. Additionally, differences in length are also noted. These evolutionary changes could account for the different kinetics of NLC between prestin species and for proteins that do not have NLC at all. In hindsight, previous research only suggested that the loop and nearby residues only limited the effective working voltage range based on the mutations introduced [53, 70]. These studies did not consider the possible outcome caused by cumulative effects for all of the mutations, whereas, deleting the entire loop provided the functional insight. In light of these results, the data presents here shows that the C-terminus is part of prestin's voltage sensor. Classic understanding of prestin's function limits charge movement and conformational changes to the membrane. From data presented herein, it is clear that the models of prestin in the literature need to be reevaluated.

These results redefine the functional role of the C-terminus as part of prestin's voltage sensor. Perhaps, this knowledge will also force us to reevaluate whether we can distinguish between prestin's membrane function and cytosolic events. In particular, the idea of a cytosolic component to the voltage sensor will likely shed light on previous studies concerning the importance of intracellular anions to prestin function $[139,141$, $142]$.

\section{6 'MARS' Model Incorporating the C-terminus with the Area Motor Model}

Characterization of prestin's C-terminus showed that it behaves as a monomer in solution and that it can assume different conformations. It was concluded that reversible interaction between the STAS A and B subdomains could be the mechanism underlying the conformations. It is possible that the 65-residue loop tethering the A and B together could modulate the interaction. The same loop was shown to be a functioning part of prestin's voltage sensor. Here, the 'MARS' (membrane-area-relay-switch) model is proposed to summarize these conclusions and suggest how they may work in the context of prestin in the cell (see Figure 6-2). In solid-state electronics, a relay switch is a device that can open and closes under the control of an electrical circuit. Here, open and close, corresponds to prestin's two states, which are expanded or contracted. The C-terminus 

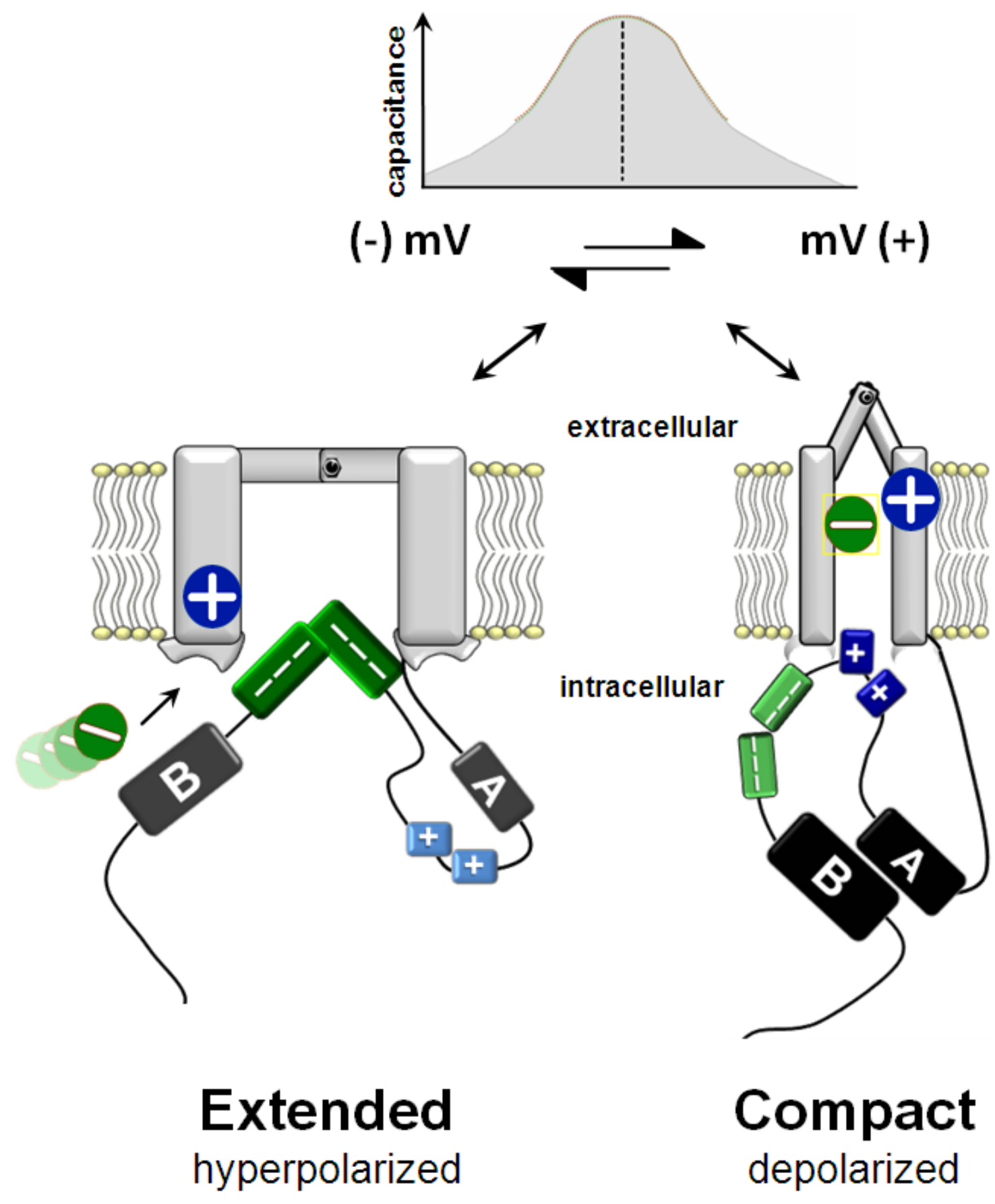

Figure 6-2 MARS model of prestin 'area' changes in the membrane

'MARS' stands for 'Membrane Area Relay Switch'. The model builds upon data showing the importance of anions and chloride to NLC. Voltage changes affect the nature of the charge interaction between the C-terminus loop and the membrane. The two green rectangles represent residues 608-613 found in the loop which are a string of six acidic residues. Chloride binding displaces $608-613$. The blue squares are basic residues at 571580. The green sphere is chloride. The blue sphere is the charge transferred through the membrane during conformational changes. 
represents part of the switch. The different conformations of the $\mathrm{C}$-terminus are the relay mechanism that supports prestin to switching between states. In terms of a cycle, hyperpolarized potentials would draw the loops negative charges (residues 608-613) into the membrane. This could separate or spread the membrane's area akin to the elongated conformation. Chloride or binding of another anion would displace these residues. Depolarization makes the inside of the cell more positive, which would pull the negatively charged residues away from the membrane and draw positive charges in the loop (possible residues 571-580) inward. Interface of the positive charges could attract the negative charges on the lipid molecules. Essentially, this would facilitate shrinking of the membrane's area. Figure 6-1 shows that the loop actually has positive and negative charges partitioned to different spots. Direct interaction with the plasma membrane is not shown for simplicity. Thus, changes to the membrane potential would allow switching between negative or positive charge interactions with the membrane. This could explain why the data presented here identified a voltage sensor function. The 571-635 deletion produced cumulative effects that diminished the way the C-terminus could interact with the membrane regardless of the potential. Other studies could not see this effect because they mutated much smaller areas of charge, which meant that only half of process was observed $[53,70]$. Deletion of the entire loop produced the greater affect.

Part of the model includes binding of chloride or another anion. Chloride is important for prestin function. Concentration-dependent reduction of chloride causes $\mathrm{V}_{\text {peak }}$ to shift in the positive direction, while diminishing NLC. It is possible that chloride's importance could be to bind near the membrane as the potential changes. Binding could then displace residues 608-613 from the membrane. In the absence of chloride, charge interactions involving the loop (608-613) could be favored to such an extent that only an extremely positive membrane potential would be able to overcome that interaction. A similar phenomenon could occur with the following two examples. A positive shift to $\mathrm{V}_{\text {peak }}$ can be observed by mutating residues R571, R572, K573, R576, $\mathrm{K} 577$, and K580 to aspartic acids. Essentially, the mutations swap positive for negative charges, which causes a positive or depolarizing shift to the operational voltage. The prestin 499 mutant significantly reduced NLC and electromotility. The mutation also appeared to cause an extreme positive shift to the membrane potential [33].

In conclusion, these studies demonstrated that prestin's C-terminus has a disordered loop that is associated with a voltage sensor function. Biochemical characterization showed that the C-terminus does not form oligomers; thus, it is unlikely that the C-terminus has a function related to prestin-prestin interaction. Finally, the STAS domain consists of two subdomains that can interact reversibly suggesting a modular role that underlies the voltage sensor function. 


\subsection{Future Studies of the Mechanism Behind the Voltage Sensor with Loop}

\subsubsection{Loop Chimaeras to Identify Functional Differences between Species}

Orthologs of prestin have been identified in several non-mammalian species to include Danio, Drosophila, and Anopheles. Expression of prestin also occurs in the hairs cells of these organisms [143]. Mammalian species of prestin exhibit distinct functional difference with the non-mammalian orthologues. Zebrafish and chicken prestin are electrogenic antiporters that transport chloride through exchange with sulfate or oxalate [144]. In zebrafish, prestin generates NLC; however, it does result in electromotility. The charge movement appears to have only weak voltage dependence, which indicates that structural differences exist with mammalian prestin. Additionally, the NLC curve is very positive. Gating currents have slow relaxation kinetics [139]. A recent report introduced point mutations to several charged residues located in the transmembrane segments. The study concluded that the residues constituted prestin's voltage based on the increased $\alpha-$ values [136]. Interestingly, the same residues identified with prestin's voltage sensor can be found in prestin orthologs and in the SLC26A6 protein. SLC26A6 does not have voltage-dependent function nor does it exhibit gating currents. Although the conclusions are not in dispute, the study was not able to explain the functional difference. The mammalian SLC26 proteins have at least 76\% identity [43], which suggests that identical or similar residues in SLC26A6 and prestin should have similar function. The loop sequence is quite divergent between prestin species in comparison to the transmembrane voltage sensor residues. Therefore, it is proposed that the charge composition and length difference between mammalian and non-mammalian prestin loops determines the functional capacity. To test this idea, prestin 'loop' chimaeras would be created, expressed in HEK 293T cells, and assayed for NLC. Residues 571-635 from mouse prestin would replace the same sequence in zebrafish and drosophila, based on the alignment in Figure 6-1. Conversely, the loop sequence from zebrafish and drosophila would replace the loop in mouse prestin.

\subsubsection{Relationship between Charged Residues in the Loop and Chloride}

Several questions remain concerning residues 571-635 identified as a disordered loop in this study. First, it is not clear why previous studies that mutated various residues in the loop region did not change the $\alpha$-value $[70,112]$. A simple explanation may come from could come the fact that the entire complement of charged residues was not explored. Residues D596, K594, E598, D600, E602, K606, K622, E627, E628, R631, and E637 were not considered. Only, the charge clusters between 571-580 and 608-613 were mutated (see Figure 6-1). To determine the residues in the loop that contribute to the voltage sensor, the charged residues mentioned need to be nullified by point mutation. These proteins would be expressed in HEK 293T cells and tested for NLC. Second, both the disorder prediction and sequence alignment hint that the loop may cover sequence beyond 571-635 (see Figure 1-6 and Figure 1-7). To refine the loop's boundaries in fulllength prestin, several three-residue deletions would be introduced systematically on each end of the loop. These proteins would be expressed in HEK 293T cells and tested for 
NLC. Finally, prestin based charge movement requires the presence of chloride [141]. Accordingly, prestin has been shown to transport chloride and several other ions [56]. It is possible to uncouple NLC from prestin transport of chloride by truncating the Cterminus to residue 709 . The truncation eliminates NLC [68, 69]; however, it does not affect chloride transport in comparison with wild type prestin [136]. This seems to conflict with the apparent requirement for the presence of chloride and NLC. It may be helpful to study chloride's role in relationship to the voltage sensor to unravel the apparent complexity. This could be studied by manipulating the intracellular or extracellular chloride during NLC measurements of the loop deletion protein in transfected cells. Varying the chloride amount would provide insight concerning possible transport function and concentration-dependence of the loop's voltage sensor function. 


\section{List of References}

1. Moller, A., Auditory physiology. 1983: Academic Press New York, New York.

2. Dallos, P., Popper, A., and Fay, R., The cochlea. 1996: Springer, New York.

3. Geisler, C., From sound to synapse: Physiology of the mammalian ear. 1998: Oxford University Press, New York.

4. Ladhams, A. and Pickles, J.O., Morphology of the monotreme organ of Corti and macula lagena. J Comp Neurol, 1996. 366(2): 335-47.

5. Manley, G.A., Cochlear mechanisms from a phylogenetic viewpoint. Proc Natl Acad Sci U S A, 2000. 97(22): 11736-43.

6. Manley, G.A. and Koppl, C., Phylogenetic development of the cochlea and its innervation. Curr Opin Neurobiol, 1998. 8(4): 468-74.

7. Manoussaki, D., Dimitriadis, E.K., and Chadwick, R.S., Cochlea's graded curvature effect on low frequency waves. Phys Rev Lett, 2006. 96(8): 88701.

8. Anniko, M. and Wroblewski, R., Ionic environment of cochlear hair cells. Hear Res, 1986. 22: 279-93.

9. Chertoff, M.E. and Brownell, W.E., Characterization of cochlear outer hair cell turgor. Am J Physiol, 1994. 266(2 Pt 1): C467-79.

10. Kachar, B., et al., Electrokinetic shape changes of cochlear outer hair cells. Nature, 1986. 322(6077): 365-8.

11. Brownell, W.E., et al., Micro- and nanomechanics of the cochlear outer hair cell. Annu Rev Biomed Eng, 2001. 3: 169-94.

12. Kalinec, F., et al., A membrane-based force generation mechanism in auditory sensory cells. PNAS, 1992. 89(18): 8671-75.

13. Holley, M.C. and Ashmore, J.F., A cytoskeletal spring in cochlear outer hair cells. Nature, 1988. 335(6191): 635-7.

14. Holley, M.C. and Ashmore, J.F., Spectrin, actin and the structure of the cortical lattice in mammalian cochlear outer hair cells. J Cell Sci, 1990. 96(Pt 2): 283-91.

15. Ashmore, J., Cochlear outer hair cell motility. Physiol Rev, 2008. 88(1): 173-210.

16. Evans, E.F., Auditory processing of complex sounds: An overview. Philos Trans R Soc Lond B Biol Sci, 1992. 336(1278): 295-306.

17. Davis, H., An active process in cochlear mechanics. Hear Res, 1983. 9(1): 79-90.

18. Evans, E.F., Place and time coding of frequency in the peripheral auditory system: Some physiological pros and cons. Audiology, 1978. 17(5): 369-420.

19. Eldredge, D.H. and Miller, J.D., Physiology of hearing. Annu Rev Physiol, 1971. 33: $281-310$.

20. von Bekesy, G., Travelling waves as frequency analysers in the cochlea. Nature, 1970. 225(5239): 1207-9.

21. Huxley, A.F., Is resonance possible in the cochlea after all? Nature, 1969. 221(5184): 935-40.

22. Rhode, W.S., Cochlear mechanics. Annu Rev Physiol, 1984. 46: 231-46.

23. Oghalai, J.S., The cochlear amplifier: Augmentation of the traveling wave within the inner ear. Curr Opin Otolaryngol Head Neck Surg, 2004. 12(5): 431-8.

24. Gold, T. and Pumphrey, R.J., Hearing. I. The cochlea as a frequency analyzer. Proceedings of the Royal Society of London. Series B - Biological Sciences, 1948. 135(881): 462-91. 
25. Brownell, W.E., Microscopic observation of cochlear hair cell motility. Scan Electron Microsc, 1984(Pt 3): 1401-6.

26. Brownell, W.E., et al., Evoked mechanical responses of isolated cochlear outer hair cells. Science, 1985. 227(4683): 194-96.

27. Dallos, P. and Corey, M.E., The role of outer hair cell motility in cochlear tuning. Curr Opin Neurobiol, 1991. 1(2): 215-20.

28. Dallos, P. and Harris, D., Properties of auditory nerve responses in absence of outer hair cells. J Neurophysiol, 1978. 41(2): 365-83.

29. Ryan, A., Dallos, P., and McGee, T., Psychophysical tuning curves and auditory thresholds after hair cell damage in the chinchilla. J Acoust Soc Am, 1979. 66(2): 370-8.

30. Ashmore, J.F., A fast motile response in guinea-pig outer hair cells: The cellular basis of the cochlear amplifier. J. Physiol., 1987. 388(1): 323-47.

31. Robles, L. and Ruggero, M.A., Mechanics of the mammalian cochlea. Physiol Rev, 2001. 81(3): 1305-52.

32. Jia, S. and He, D.Z., Motility-associated hair-bundle motion in mammalian outer hair cells. Nat Neurosci, 2005. 8(8): 1028-34.

33. Dallos, P., et al., Prestin-based outer hair cell motility is necessary for mammalian cochlear amplification. Neuron, 2008. 58(3): 333-9.

34. Fettiplace, R., Active hair bundle movements in auditory hair cells. J Physiol, 2006. 576(Pt 1): 29-36.

35. Corey, D.P., What is the hair cell transduction channel? J Physiol, 2006. 576(Pt 1): 23-8.

36. Iwasa, K.H., A two-state piezoelectric model for outer hair cell motility. Biophys J, 2001. 81(5): 2495-506.

37. Dong, X.X., Ospeck, M., and Iwasa, K.H., Piezoelectric reciprocal relationship of the membrane motor in the cochlear outer hair cell. Biophys J, 2002. 82(3): 1254-9.

38. Holley, M.C. and Ashmore, J.F., On the mechanism of a high-frequency force generator in outer hair cells isolated from the guinea pig cochlea. Proc R Soc Lond B Biol Sci, 1988. 232(1269): 413-29.

39. Iwasa, K.H. and Adachi, M., Force generation in the outer hair cell of the cochlea. Biophys J, 1997. 73(1): 546-55.

40. Hudspeth, A., Mechanical amplification of stimuli by hair cells. Curr Opin Neurobiol, 1997. 7(4): 480-6.

41. Iwasa, K.H., A membrane motor model for the fast motility of the outer hair cell. J Acoust Soc Am, 1994. 96(4): 2216-24.

42. Zheng, J., et al., Prestin is the motor protein of cochlear outer hair cells. Nature, 2000. 405(6783): 149-55.

43. Mount, D.B. and Romero, M.F., The SLC26 gene family of multifunctional anion exchangers. Pflugers Arch, 2004. 447(5): 710-21.

44. Dallos, P., Hallworth, R., and Evans, B.N., Theory of electrically driven shape changes of cochlear outer hair cells. J Neurophysiol, 1993. 70(1): 299-323.

45. Kakehata, S. and Santos-Sacchi, J., Effects of salicylate and lanthanides on outer hair cell motility and associated gating charge. J. Neurosci., 1996. 16(16): 488189. 
46. Jewett, D.L., Romano, M.N., and Williston, J.S., Human auditory evoked potentials: Possible brain stem components detected on the scalp. Science, 1970. 167(924): 1517-8.

47. Kemp, D.T., Stimulated acoustic emissions from within the human auditory system. J Acoust Soc Am, 1978. 64(5): 1386-91.

48. Liberman, M.C., et al., Prestin is required for electromotility of the outer hair cell and for the cochlear amplifier. Nature, 2002. 419(6904): 300-4.

49. Dallos, P., Evans, B.N., and Hallworth, R., Nature of the motor element in electrokinetic shape changes of cochlear outer hair cells. Nature, 1991. 350(6314): 155-7.

50. Huang, G. and Santos-Sacchi, J., Motility voltage sensor of the outer hair cell resides within the lateral plasma membrane. Proc Natl Acad Sci U S A, 1994. 91(25): 12268-72.

51. Okamura, Y., Biodiversity of voltage sensor domain proteins. Pflugers Arch, 2007. 454(3): 361-71.

52. Bezanilla, F., How membrane proteins sense voltage. Nat Rev Mol Cell Biol, 2008. 9(4): 323-32.

53. Oliver, D., et al., Intracellular anions as the voltage sensor of prestin, the outer hair cell motor protein. Science, 2001. 292(5525): 2340-3.

54. Okoruwa, O.E., et al., Evolutionary insights into the unique electromotility motor of mammalian outer hair cells. Evol Dev, 2008. 10(3): 300-15.

55. Oliver, D., Schachinger, T., and Fakler, B., Interaction of prestin (SLC26A5) with monovalent intracellular anions. Novartis Found Symp, 2006. 273: 244-53; discussion 53-60, 61-4.

56. Muallem, D. and Ashmore, J., An anion antiporter model of prestin, the outer hair cell motor protein. Biophys J, 2006. 90(11): 4035-45.

57. Adachi, M. and Iwasa, K.H., Effect of diamide on force generation and axial stiffness of the cochlear outer hair cell. Biophys J, 1997. 73(5): 2809-18.

58. Oghalai, J.S., et al., Fluorescence-imaged microdeformation of the outer hair cell lateral wall. J Neurosci, 1998. 18(1): 48-58.

59. Shehata, W.E., Brownell, W.E., and Dieler, R., Effects of salicylate on shape, electromotility and membrane characteristics of isolated outer hair cells from guinea pig cochlea. Acta Otolaryngol, 1991. 111(4): 707-18.

60. Tunstall, M.J., Gale, J.E., and Ashmore, J.F., Action of salicylate on membrane capacitance of outer hair cells from the guinea-pig cochlea. J Physiol, 1995. 485(3): 739-52.

61. Lue, A.J. and Brownell, W.E., Salicylate induced changes in outer hair cell lateral wall stiffness. Hear Res, 1999. 135(1-2): 163-8.

62. Drexl, M., et al., The role of prestin in the generation of electrically evoked otoacoustic emissions in mice. J Neurophysiol, 2008. 99(4): 1607-15.

63. Gulley, R.L. and Reese, T.S., Regional specialization of the hair cell plasmalemma in the organ of Corti. Anat Rec, 1977. 189(1): 109-23.

64. Forge, A., Structural features of the lateral walls in mammalian cochlear outer hair cells. Cell Tissue Res, 1991. 265(3): 473-83.

65. Murakoshi, M. and Wada, H., Atomic force microscopy in studies of the cochlea. Methods Mol Biol, 2009. 493: 401-13. 
66. Mio, K., et al., The motor protein prestin is a bullet-shaped molecule with inner cavities. J Biol Chem, 2008. 283(2): 1137-45.

67. Zheng, J., et al., Analysis of the oligomeric structure of the motor protein prestin. J Biol Chem, 2006. 281(29): 19916-24.

68. Navaratnam, D., et al., N-terminal-mediated homomultimerization of prestin, the outer hair cell motor protein. Biophys J, 2005. 89(5): 3345-52.

69. Zheng, J., et al., The c-terminus of prestin influences nonlinear capacitance and plasma membrane targeting. J Cell Sci, 2005. 118(Pt 13): 2987-96.

70. Bai, J.P., et al., En block c-terminal charge cluster reversals in prestin (SLC26A5): Effects on voltage-dependent electromechanical activity. Neurosci Lett, 2006. 404(3): 270-5.

71. Saier, M.H., Jr., et al., Phylogenetic characterization of novel transport protein families revealed by genome analyses. Biochim Biophys Acta, 1999. 1422(1): 156.

72. Dawson, P.A. and Markovich, D., Pathogenetics of the human SLC26 transporters. Curr Med Chem, 2005. 12(4): 385-96.

73. Zheng, J., et al., Prestin topology: Localization of protein epitopes in relation to the plasma membrane. Neuroreport, 2001. 12(9): 1929-35.

74. Aravind, L. and Koonin, E.V., The STAS domain - a link between anion transporters and antisigma-factor antagonists. Curr Biol, 2000. 10(2): R53-5.

75. Dorwart, M.R., et al., Congenital chloride-losing diarrhea causing mutations in the STAS domain result in misfolding and mistrafficking of SLC26A3. J Biol Chem, 2008. 283(13): 8711-22.

76. Dorwart, M.R., et al., The solute carrier 26 family of proteins in epithelial ion transport. Physiology (Bethesda), 2008. 23: 104-14.

77. Shcheynikov, N., et al., Regulatory interaction between CFTR and the SLC26 transporters. Novartis Found Symp, 2006. 273: 177-86; discussion 86-92, 261-4.

78. Rouached, H., et al., Structural and functional analysis of the c-terminal STAS (sulfate transporter and anti-sigma antagonist) domain of the Arabidopsis thaliana sulfate transporter Sultr1;2. J Biol Chem, 2005. 280(16): 15976-83.

79. Shibagaki, N. and Grossman, A.R., The role of the STAS domain in the function and biogenesis of a sulfate transporter as probed by random mutagenesis. J Biol Chem, 2006. 281(32): 22964-73.

80. Shibagaki, N. and Grossman, A.R., Probing the function of STAS domains of the Arabidopsis sulfate transporters. J Biol Chem, 2004. 279(29): 30791-9.

81. Everett, L.A., et al., Pendred syndrome is caused by mutations in a putative sulphate transporter gene (PDS). Nat Genet, 1997. 17(4): 411-22.

82. Karniski, L.P., Functional expression and cellular distribution of diastrophic dysplasia sulfate transporter (DTDST) gene mutations in hek cells. Hum Mol Genet, 2004. 13(19): 2165-71.

83. Park, H.J., et al., Origins and frequencies of SLC26A4 (PDS) mutations in east and south asians: Global implications for the epidemiology of deafness. J Med Genet, 2003. 40(4): 242-8.

84. Makela, S., et al., SLC26A3 mutations in congenital chloride diarrhea. Hum Mutat, 2002. 20(6): 425-38. 
85. Seavers, P.R., et al., Structure of the Bacillus cell fate determinant SpoIIaa in phosphorylated and unphosphorylated forms. Structure, 2001. 9(7): 605-14.

86. Ha, K.S., et al., Crystallization and preliminary $x$-ray crystallographic analysis of the TM1442 gene product from Thermotoga maritima, a homologue of Bacillus subtilis anti-anti-sigma factors. Acta Crystallogr D Biol Crystallogr, 2001. 57(Pt 2): 276-8.

87. Clarkson, J., Campbell, I.D., and Yudkin, M.D., NMR studies of the interactions of SpoIIaa with its partner proteins that regulate sporulation in Bacillus subtilis. $\mathrm{J}$ Mol Biol, 2001. 314(3): 359-64.

88. Masuda, S., et al., Crystal structures of the ADP and ATP bound forms of the Bacillus anti-sigma factor SpoIIab in complex with the anti-anti-sigma SpoIIaa. J Mol Biol, 2004. 340(5): 941-56.

89. Najafi, S.M., Harris, D.A., and Yudkin, M.D., The SpoIIaa protein of Bacillus subtilis has GTP-binding properties. J Bacteriol, 1996. 178(22): 6632-4.

90. Grishin, N.V., Fold change in evolution of protein structures. J Struct Biol, 2001. 134(2-3): 167-85.

91. Krishna, S.S. and Grishin, N.V., Structural drift: A possible path to protein fold change. Bioinformatics, 2005. 21(8): 1308-10.

92. Pirovano, W., Feenstra, K.A., and Heringa, J., The meaning of alignment: Lessons from structural diversity. BMC Bioinformatics, 2008. 9: 556.

93. McGuffin, L.J., Bryson, K., and Jones, D.T., The PSIPRED protein structure prediction server. Bioinformatics, 2000. 16(4): 404-5.

94. Pollastri, G., et al., Improving the prediction of protein secondary structure in three and eight classes using recurrent neural networks and profiles. Proteins, 2002. 47(2): 228-35.

95. Cole, C., Barber, J.D., and Barton, G.J., The Jpred 3 secondary structure prediction server. Nucleic Acids Res, 2008. 36: W197-201.

96. Bennett-Lovsey, R.M., et al., Exploring the extremes of sequence/structure space with ensemble fold recognition in the program Phyre. Proteins, 2008. 70(3): 61125.

97. Pasqualetto, E., et al., Expression, purification and characterisation of the cterminal STAS domain of the SLC26 anion transporter prestin. Protein Expr Purif, 2008. 58(2): 249-56.

98. Kovacs, H., et al., NMR studies of the sporulation protein SpoIIaa: Implications for the regulation of the transcription factor sigmaF in Bacillus subtilis. J Biomol NMR, 2001. 19(4): 293-304.

99. Thompson, J.D., Gibson, T.J., and Higgins, D.G., Multiple sequence alignment using ClustalW and ClustalX. Curr Protoc Bioinformatics, 2002. Chapter 2: Unit 2.3 .

100. Clamp, M., et al., The Jalview Java alignment editor. Bioinformatics, 2004. 20(3): 426-7.

101. Obradovic, Z., et al., Exploiting heterogeneous sequence properties improves prediction of protein disorder. Proteins, 2005. 61(S7): 176-82.

102. Romero, P., et al., Sequence complexity of disordered protein. Proteins, 2001. 42(1): 38-48.

103. Birney, E., et al., An overview of Ensembl. Genome Res, 2004. 14(5): 925-8. 
104. Bertani, G., Lysogeny at mid-twentieth century: P1, P2, and other experimental systems. J Bacteriol, 2004. 186(3): 595-600.

105. Schuck, P., Size-distribution analysis of macromolecules by sedimentation velocity ultracentrifugation and lamm equation modeling. Biophys J, 2000. 78(3): 1606-19.

106. Brown, P.H. and Schuck, P., Macromolecular size-and-shape distributions by sedimentation velocity analytical ultracentrifugation. Biophys J, 2006. 90(12): 4651-61.

107. Schuck, P., On the analysis of protein self-association by sedimentation velocity analytical ultracentrifugation. Anal Biochem, 2003. 320(1): 104-24.

108. Vistica, J., et al., Sedimentation equilibrium analysis of protein interactions with global implicit mass conservation constraints and systematic noise decomposition. Anal Biochem, 2004. 326(2): 234-56.

109. Kendrick, B.S., et al., Online size-exclusion high-performance liquid chromatography light scattering and differential refractometry methods to determine degree of polymer conjugation to proteins and protein-protein or protein-ligand association states. Anal Biochem, 2001. 299(2): 136-46.

110. Gietz, R.D. and Schiestl, R.H., High-efficiency yeast transformation using the LiAc/SS carrier DNA/peg method. Nat Protoc, 2007. 2(1): 31-4.

111. Dallos, P. and Fakler, B., Prestin, a new type of motor protein. Nat Rev Mol Cell Biol, 2002. 3(2): 104-11.

112. Deak, L., et al., Effects of cyclic nucleotides on the function of prestin. J Physiol, 2005. 563(Pt 2): 483-96.

113. Yu, N., Zhu, M.L., and Zhao, H.B., Prestin is expressed on the whole outer hair cell basolateral surface. Brain Res, 2006. 1095(1): 51-8.

114. Anvari, B., et al., Effects of prestin on membrane mechanics and electromechanics. Conf Proc IEEE Eng Med Biol Soc, 2007. 2007: 5384-6.

115. Levitt, M. and Chothia, C., Structural patterns in globular proteins. Nature, 1976. 261(5561): 552-8.

116. Receveur-Brechot, V., et al., Assessing protein disorder and induced folding. Proteins, 2006. 62(1): 24-45.

117. Kriwacki, R.W., et al., Probing protein structure using biochemical and biophysical methods. Proteolysis, matrix-assisted laser desorption/ionization mass spectrometry, high-performance liquid chromatography and size-exclusion chromatography of p21Waf1/Cip1/Sdil. J Chromatogr A, 1997. 777(1): 23-30.

118. Markus, G., Protein substrate conformation and proteolysis. Proc Natl Acad Sci U S A, 1965. 54(1): 253-8.

119. Beynon, R. and Bond, J., Proteolytic enzymes: A practical approach. 2001: Oxford University Press, New York.

120. Ottesen, M. and Svendsen, I., The subtilisins. Methods in enzymology, 1970. 19: 199-215.

121. Harding, S., Rowe, A., and Horton, J., Analytical ultracentrifugation in biochemistry and polymer science. 1992: Royal Society of Chemistry Cambridge, England. 
122. Brown, P.H., Balbo, A., and Schuck, P., Characterizing protein-protein interactions by sedimentation velocity analytical ultracentrifugation. Curr Protoc Immunol, 2008. Chapter 18: Unit 18.15.

123. Dyson, H.J. and Wright, P.E., Nuclear magnetic resonance methods for elucidation of structure and dynamics in disordered states. Methods Enzymol, 2001. 339: 258-70.

124. Dyson, H.J. and Wright, P.E., Equilibrium NMR studies of unfolded and partially folded proteins. Nat Struct Biol, 1998. 5(S7): 499-503.

125. le Maire, M., Viel, A., and Moller, J.V., Size exclusion chromatography and universal calibration of gel columns. Anal Biochem, 1989. 177(1): 50-6.

126. Attri, A.K. and Minton, A.P., New methods for measuring macromolecular interactions in solution via static light scattering: Basic methodology and application to nonassociating and self-associating proteins. Anal Biochem, 2005. 337(1): 103-10.

127. Rich, R.L., et al., Kinetic analysis of estrogen receptor/ligand interactions. Proc Natl Acad Sci U S A, 2002. 99(13): 8562-7.

128. Galea, C.A., et al., Role of intrinsic flexibility in signal transduction mediated by the cell cycle regulator, p27 Kip1. J Mol Biol, 2008. 376(3): 827-38.

129. Buck, M., Xu, W., and Rosen, M.K., A two-state allosteric model for autoinhibition rationalizes wasp signal integration and targeting. J Mol Biol, 2004. 338(2): 271-85.

130. Orengo, C.A. and Thornton, J.M., Protein families and their evolution-a structural perspective. Annu Rev Biochem, 2005. 74: 867-900.

131. Fontana, A., et al., Probing protein structure by limited proteolysis. Acta Biochim Pol, 2004. 51(2): 299-321.

132. Twigg, P.D., et al., Disordered to ordered folding in the regulation of diphtheria toxin repressor activity. Proc Natl Acad Sci U S A, 2001. 98(20): 11259-64.

133. Kussie, P.H., et al., Structure of the mdm2 oncoprotein bound to the p53 tumor suppressor transactivation domain. Science, 1996. 274(5289): 948-53.

134. Magidovich, E., et al., Intrinsic disorder in the c-terminal domain of the shaker voltage-activated $K+$ channel modulates its interaction with scaffold proteins. Proc Natl Acad Sci U S A, 2007. 104(32): 13022-7.

135. Wissmann, R., et al., NMR structure and functional characteristics of the hydrophilic n-terminus of the potassium channel beta-subunit Kvbeta1.1. J Biol Chem, 1999. 274(50): 35521-5.

136. Bai, J.P., et al., Prestin's anion transport and voltage-sensing capabilities are independent. Biophys J, 2009. 96(8): 3179-86.

137. Ko, S.B., et al., Gating of CFTR by the STAS domain of SLC26 transporters. Nat Cell Biol, 2004. 6(4): 343-50.

138. Ostedgaard, L.S., et al., A functional $r$ domain from cystic fibrosis transmembrane conductance regulator is predominantly unstructured in solution. Proc Natl Acad Sci U S A, 2000. 97(10): 5657-62.

139. Albert, J.T., et al., Voltage-sensitive prestin orthologue expressed in zebrafish hair cells. J Physiol, 2007. 580(2): 451-61. 
140. Franchini, L.F. and Elgoyhen, A.B., Adaptive evolution in mammalian proteins involved in cochlear outer hair cell electromotility. Mol Phylogenet Evol, 2006. 41(3): 622-35.

141. Santos-Sacchi, J., et al., Control of mammalian cochlear amplification by chloride anions. J Neurosci, 2006. 26(15): 3992-8.

142. Rybalchenko, V. and Santos-Sacchi, J., Cl-flux through a non-selective, stretchsensitive conductance influences the outer hair cell motor of the guinea-pig. $\mathrm{J}$ Physiol, 2003. 547(Pt 3): 873-91.

143. Weber, T., et al., Expression of prestin-homologous solute carrier (SLC26) in auditory organs of nonmammalian vertebrates and insects. Proc Natl Acad Sci U S A, 2003. 100(13): 7690-5.

144. Schaechinger, T.J. and Oliver, D., Nonmammalian orthologs of prestin (SLC26A5) are electrogenic divalent/chloride anion exchangers. Proc Natl Acad Sci U S A, 2007. 104(18): 7693-8. 


\section{Appendix A Figures of Ear Anatomy}

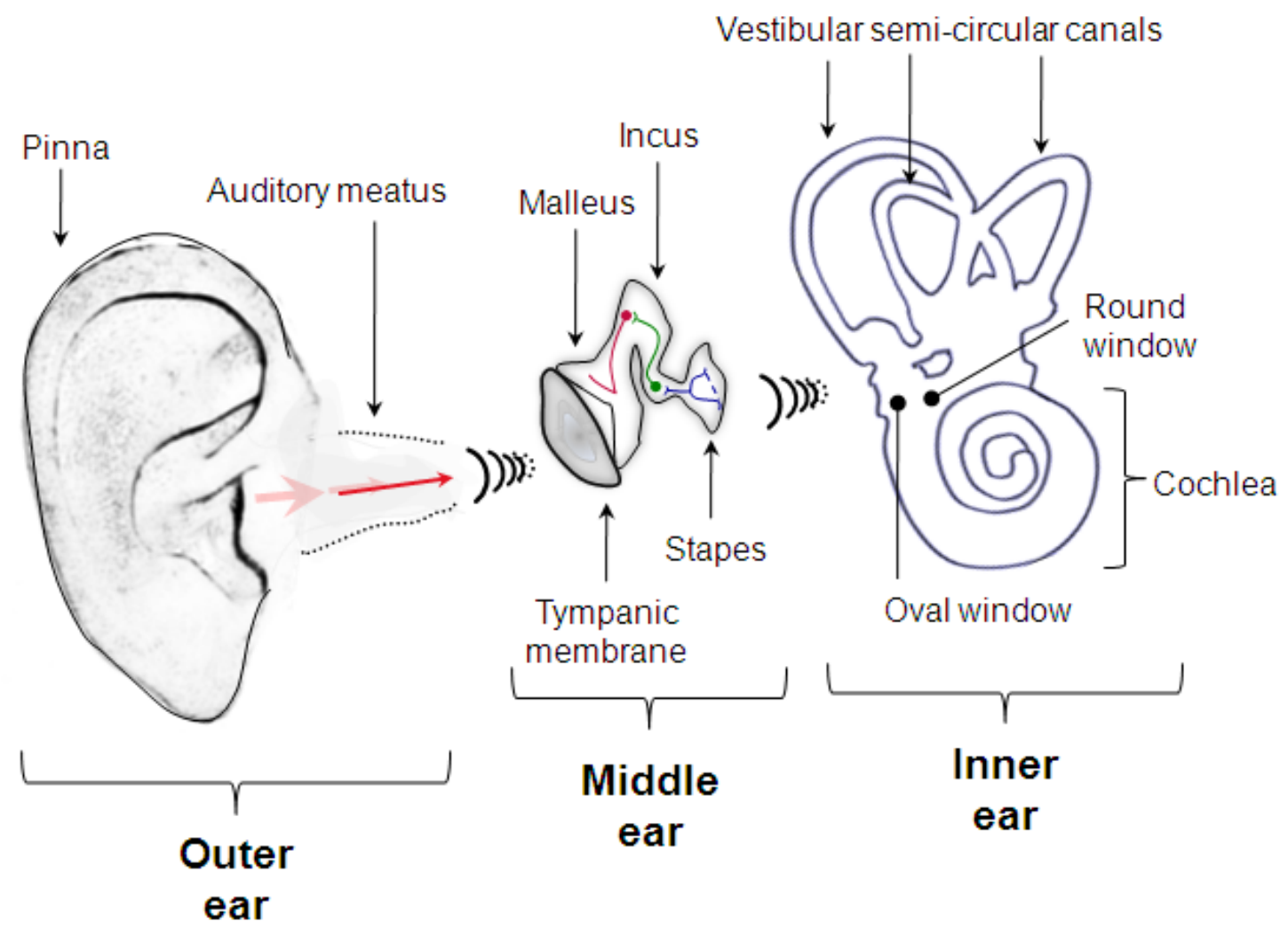

Figure A-1 Pathway of sound into the cochlea

Depicted are the three main anatomical and functional areas in a human ear. Together, they serve as a conduit that channels acoustic energy to the OHCs cells where prestin is located. 


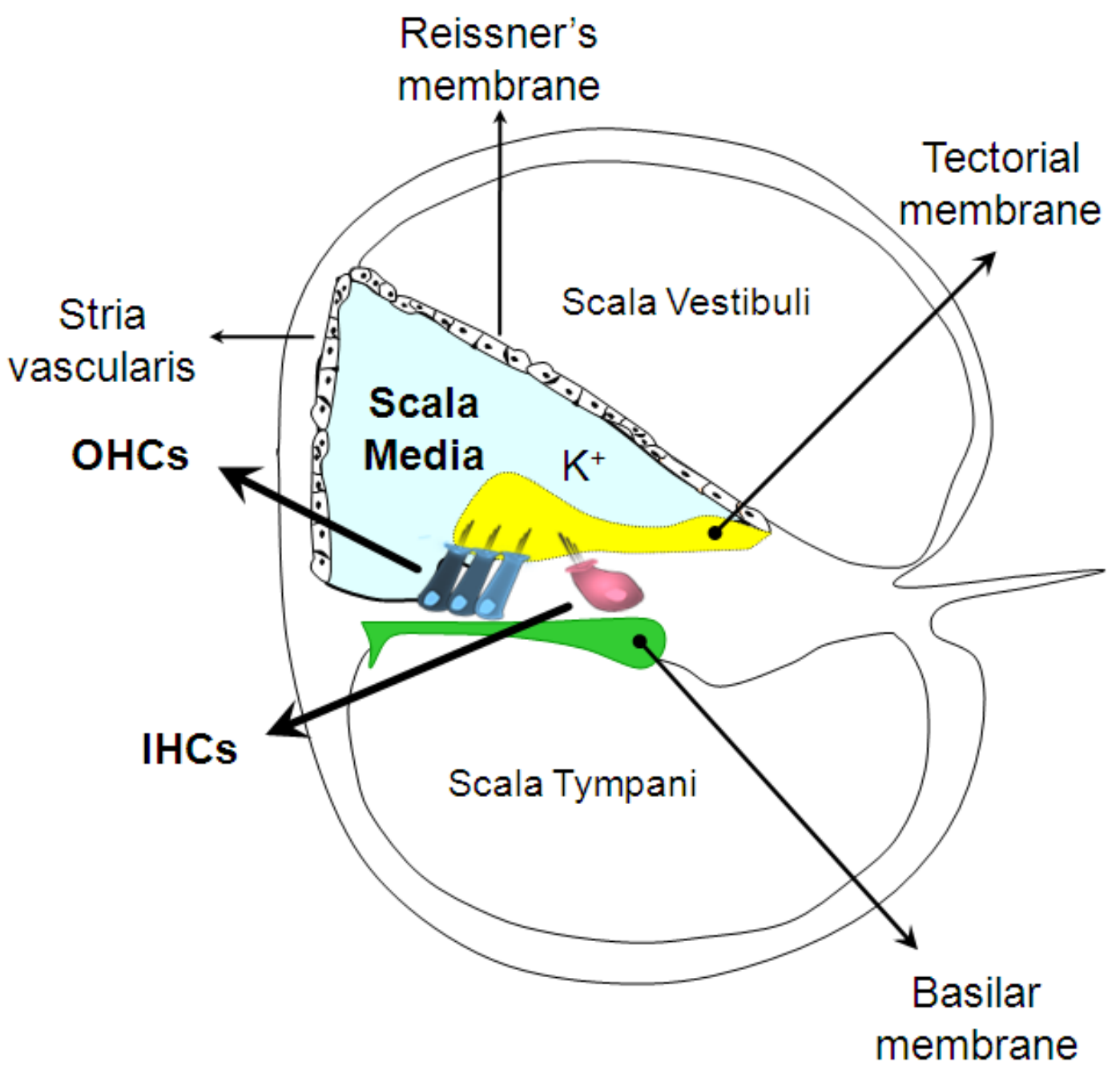

Figure A-2 Cross-section of the cochlea showing the key sensory structures Only a single turn of the cochlear spiral is shown. The organ of Corti is composed of the OHCs (blue), IHCs (pink), tectorial membrane (yellow), and the basilar membrane (green). The tunnel of Corti is a fluid channel (white space) that resides between the two types of hair cells. Many of the cellular structures associated with the OHCs and the IHCs have been omitted for simplicity. The stria vascularis maintains the high potassium content in the scala media which serves as a 'battery' to power hair cell depolarization. Reissner's membrane and the reticular plate (not shown) seal the scala media from the scala vestibuli and tympani maintaining the potassium level. Functional integration between the $\mathrm{OHCs}$, IHCs, the basilar membrane, and the tectorial membrane (yellow) underlies signal transduction. 


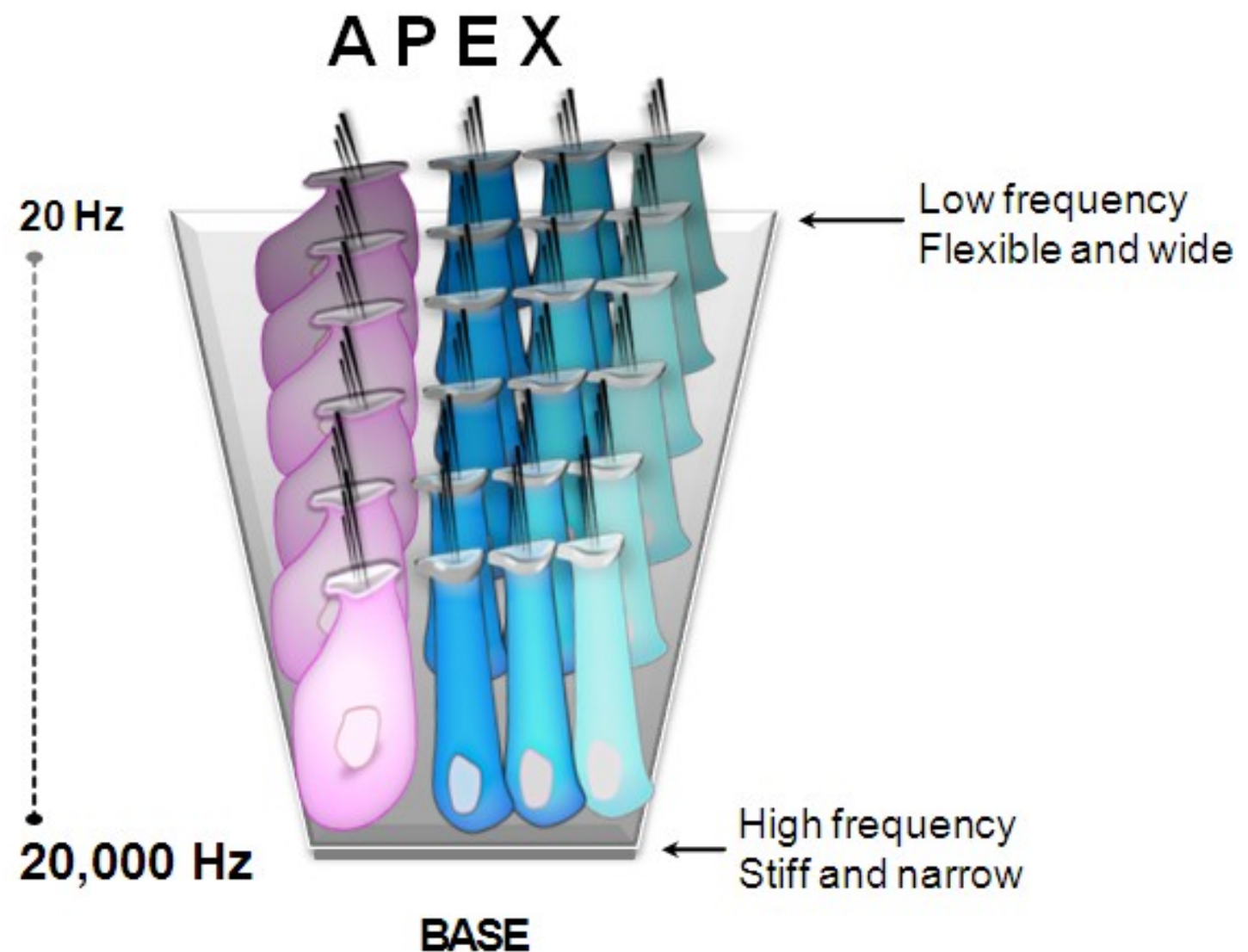

Figure A-3 Tonotopic layout of the hair cells along the basilar membrane

The basilar membrane exhibits a graded stiffness along its length. The measure of flexibility equates to resonance at particular frequencies. Similarly, the hair cells exhibit frequency specific activity based on their location along the basilar membrane. The hair cells also differ slightly in height. Basal hair cells are longer than apical cells. The frequency range shown applies to a young human adult. IHCs are on the left in pink. $\mathrm{OHCs}$ are on the right in blue. 


\section{Appendix B Buffers, Solutions, and Media}

$\underline{\text { LB (Lysogeny Broth [104]) Miller media }}$

Peptone from casein $10 \mathrm{~g}$

Sodium chloride $\quad 10 \mathrm{~g}$

Yeast extract $5 \mathrm{~g}$

Antibiotic (ampicillin $200 \mu \mathrm{g}+$ chloramphenicol $30 \mu \mathrm{g}$ or kanamycin $30 \mu \mathrm{g}$ )

milli-Q water to 1 liter

Glutathione sepharose binding buffer

$50 \mathrm{mM}$ Tris $\mathrm{pH} 7.4$

$200 \mathrm{mM} \mathrm{NaCl}$

$5 \mathrm{mM}$ DTT

Ni-NTA binding buffer

$20 \mathrm{mM}$ Tris 8.0

$10 \mathrm{mM}$ imidazole

$500 \mathrm{mM} \mathrm{NaCl}$

4X LDS gel-loading dye

$106 \mathrm{mM}$ Tris $\mathrm{HCl}$

$141 \mathrm{mM}$ Tris base

$2 \%$ LDS and 10\% Glycerol

$0.51 \mathrm{mM}$ EDTA

$0.22 \mathrm{mM}$ SERVA Blue G250

$0.175 \mathrm{mM}$ Phenol Red pH 8.5

$10 \%$ Bis-Tris gel running buffer $\mathrm{pH} 7.3$

$50 \mathrm{mM}$ MES

$50 \mathrm{mM}$ Tris base

$0.1 \%$ SDS

$1 \mathrm{mM}$ EDTA

Glutathione sepharose elution buffer

$50 \mathrm{mM}$ Tris $\mathrm{pH} 8.0$

$200 \mathrm{mM} \mathrm{NaCl}$

$5 \mathrm{mM}$ DTT

$10 \mathrm{mM}$ L-glutathione reduced

Nickel elution buffer

$20 \mathrm{mM}$ Tris $\mathrm{pH} 8.0$

$500 \mathrm{mM}$ imidazole

$500 \mathrm{mM} \mathrm{NaCl}$ 
Q sepharose or Mono Q loading buffer

$50 \mathrm{mM}$ Tris $\mathrm{pH} 7.4$

$50 \mathrm{mM} \mathrm{NaCl}$

$5 \mathrm{mM}$ DTT

Q-sepharose or Mono Q elution buffer

$50 \mathrm{mM}$ Tris $\mathrm{pH} 7.4$

$1 \mathrm{M} \mathrm{NaCl}$

$5 \mathrm{mM}$ DTT

Gel filtration buffer $\mathrm{pH} 7.0$

$15.4 \mathrm{mM} \mathrm{K}_{2} \mathrm{HPO}_{4}$

$9.6 \mathrm{mM} \mathrm{KH}_{2} \mathrm{PO}_{4}$

$150 \mathrm{mM} \mathrm{NaCl}$

$5 \mathrm{mM}$ DTT

Trypsin and subtilisin digest buffer

$50 \mathrm{mM}$ Tris $\mathrm{pH} 7.6$

$150 \mathrm{mM} \mathrm{NaCl}$

5 mM DTT

10X CAPS buffer $\mathrm{pH} 11$ (10X CAPS)

22.13 g 3-[cyclohexylamino]1-propanesulfonic acid milli-Q water to 1 liter

PVDF electroblotting transfer buffer

$200 \mathrm{ml} 10 \mathrm{X}$ CAPS buffer

$20 \%$ methanol

milli-Q water to 2 liters

PVDF membrane staining solution

$50 \mathrm{ml}$ of $1 \%$ Stock Coomassie Stain

$200 \mathrm{ml}$ methanol

$5 \mathrm{ml}$ acetic acid

milli-Q water to $500 \mathrm{ml}$

Destain Solution

$50 \%$ methanol

SV buffer $\mathrm{pH} 7.0$

$15.4 \mathrm{mM} \mathrm{K}_{2} \mathrm{HPO}_{4}$

$9.6 \mathrm{mM} \mathrm{KH}_{2} \mathrm{PO}_{4}$

$150 \mathrm{mM} \mathrm{NaCl}$

$1 \mathrm{mM}$ DTT 
$\underline{\text { SE buffer } \mathrm{pH} 7.0}$

$15.4 \mathrm{mM} \mathrm{K}_{2} \mathrm{HPO}_{4}$

$9.6 \mathrm{mM} \mathrm{KH}_{2} \mathrm{PO}_{4}$

$150 \mathrm{mM} \mathrm{NaCl}$

SEC-MALS buffer $\mathrm{pH} 7.0$

$15.4 \mathrm{mM} \mathrm{K}_{2} \mathrm{HPO}_{4}$

$9.6 \mathrm{mM} \mathrm{KH}_{2} \mathrm{PO}_{4}$

$150 \mathrm{mM} \mathrm{NaCl}$

$1 \mathrm{mM}$ DTT

SPR buffer

$10 \mathrm{mM}$ Tris $\mathrm{pH} 7.4$

$150 \mathrm{mM} \mathrm{NaCl}$

$5 \mathrm{mM}$ DTT

$0.005 \%$ Tween 20

NMR buffer $\mathrm{pH} 7.0$

$15.4 \mathrm{mM} \mathrm{K}_{2} \mathrm{HPO}_{4}$

$9.6 \mathrm{mM} \mathrm{KH}_{2} \mathrm{PO}_{4}$

$25 \mathrm{mM} \mathrm{NaCl}$

$10 \% \mathrm{D}_{2} \mathrm{O}$

Circular dichroism buffer $\mathrm{pH} 6.6$

$9.5 \mathrm{mM} \mathrm{K}_{2} \mathrm{HPO}_{4}$

$15.5 \mathrm{mM} \mathrm{KH}_{2} \mathrm{PO}_{4}$

$5 \mathrm{mM} \mathrm{NaCl}$

Tris EDTA buffer

$10 \mathrm{mM}$ Tris $\mathrm{pH} 7.5$

$1 \mathrm{mM}$ EDTA 


\begin{abstract}
Vita
Michael S. Podgorski was born in Wausau, Wisconsin on February 22, 1971. Upon completion of high school in 1989, he served in the United States Army as a Spanish linguist from 1989 to 1996. After his service ended, he earned his Bachelor of Science degree in Molecular Biology from the University of Central Florida in May 2001. He gained laboratory experience during his undergraduate studies by maintaining cultures of Plasmodium falciparum used for malaria research. Subsequently, he worked for two years as a research technician in a structural biology lab at St. Jude Children's Research Hospital in Memphis, Tennessee. In August of 2003, he began his graduate studies in an interdisciplinary science program at the University of Tennessee Health Science Center in Memphis. Michael joined Dr. Jian Zuo's laboratory at St. Jude Children's Research Hospital to carry out his dissertation work. He anticipates completion and award of a Doctor of Philosophy degree by December 2009.
\end{abstract}

